\title{
Bridging the Gap Between Physiology and Behavior: Evidence From the sSoTS Model of Human Visual Attention
}

\author{
Eirini Mavritsaki, Dietmar Heinke, and Harriet Allen \\ University of Birmingham
}

\author{
Gustavo Deco \\ Universitat Pompeu Fabra
}

\author{
Glyn W. Humphreys \\ University of Birmingham
}

\begin{abstract}
We present the case for a role of biologically plausible neural network modeling in bridging the gap between physiology and behavior. We argue that spiking-level networks can allow "vertical" translation between physiological properties of neural systems and emergent "whole-system" performanceenabling psychological results to be simulated from implemented networks and also inferences to be made from simulations concerning processing at a neural level. These models also emphasize particular factors (e.g., the dynamics of performance in relation to real-time neuronal processing) that are not highlighted in other approaches and that can be tested empirically. We illustrate our argument from neural-level models that select stimuli by biased competition. We show that a model with biased competition dynamics can simulate data ranging from physiological studies of single-cell activity (Study 1) to whole-system behavior in human visual search (Study 2), while also capturing effects at an intermediate level, including performance breakdown after neural lesion (Study 3) and data from brain imaging (Study 4). We also show that, at each level of analysis, novel predictions can be derived from the biologically plausible parameters adopted, which we proceed to test (Study 5). We argue that, at least for studying the dynamics of visual attention, the approach productively links single-cell to psychological data.
\end{abstract}

Keywords: visual search, integrate-and-fire neurons, computational model, fMRI, neuropsychology

Understanding human cognition is difficult. One of the difficulties is that cognition can be described at many different levelsfrom the high-level computational principles that may shape the landscape within which processes must function, through to the physiological principles by which neurons operate. How can these different levels best be related? Marr (1982) provided an influential framework in which different levels of analysis were described. This framework separated out, on the one hand, theories dealing with the computational principles and the neural hardware, from, on the other, "midlevel" theories that deal with abstract algorithms, which could operate on a variety of platforms (computers as well as human brains). However, what was not specified in this framework was how we move between the different levels; indeed, the levels were described as operating in a quasi-independent manner. This means that, within the framework, it is possible to have an algorithmic account of cognition that is separated from constraints

Eirini Mavritsaki, Dietmar Heinke, Harriet Allen, and Glyn W. Humphreys, School of Psychology, University of Birmingham, Birmingham, England; Gustavo Deco, Department of Technology, Computational Neuroscience, Institució Catalana de Recerca i Estudis Avançats (ICREA), Universitat Pompeu Fabra, Barcelona, Spain.

This work was supported by the Biotechnology and Biology and Medical Research Councils, United Kingdom, the EU Framework 6 (Decisions in Motion), and the Royal Society.

Correspondence concerning this article should be addressed to Glyn W. Humphreys, School of Psychology, University of Birmingham, Birmingham, B15 2TT, England. E-mail: g.w.humphreys@bham.ac.uk based on neural-level functions, as well as neural-level accounts unconstrained by data derived from the operation of component cognitive processes. This leaves unspecified how we may link theories dealing with abstract psychological mechanisms (attention, memory, decision making) to theories that are concerned with how neurons communicate. It also means that the benefits that might be gained from this linkage are unexploited; theoretical insights at one level do not infiltrate other levels. The aim of this article is to describe an approach to develop links between physiology and high-level behavior through the use of formal neural models. Our argument is illustrated by an attempt to develop a neural-level model of human visual attention, which we show can capture effects at different levels of analysis (from single-cell electrophysiology through to whole-system psychological experiments). We go on to show that the interaction between modeling and empirical studies can be a two-way process by deriving and testing new empirical predictions that emerge from the dynamic processes introduced by modeling at the neural level. We argue that formal, neural models can provide a means of unifying the levels of description outlined by Marr and that there is empirical validation for this approach when applied to human visual attention.

\section{Defining Cognitive Subsystems}

Since its inception in the 1960s, cognitive psychology has made advances in our understanding of human cognition by breaking down "whole-system" behavior into distinct components. A variety of empirical approaches have been proposed in attempts to break down whole-system behavior into underlying component 
processes_including additive factors logic (Sternberg, 1969), the psychological refractory period (Pashler, 2000), and studies of brain lesioned patients. A common aim of these approaches is to define processing in terms of a series of stages, and then to test the impact of different factors on the contrasting stages. Consider a neuropsychological example. After damage to posterior parietal cortex (PPC), patients can present with the problem of extinction; the patients are able to respond to a single stimulus presented on the side contralateral to their lesion but fail to detect the same item when another stimulus is presented simultaneously on the ipsilesional side (Karnath, 1988). This deficit can be understood in terms of the patients suffering from spatial biases when simultaneous stimuli compete for selection (Duncan, Humphreys, \& Ward, 1997). The lesion suffered by the patients biases selection in favor of stimuli appearing on the ipsilesional side. This does not have serious consequences on selection when the contralesional stimulus is the only item in the field, but it does when the contralesional stimulus must compete for selection with the ipsilesional item; in this case, the contralesional stimulus loses and is extinguished. This result is consistent with a deficit at a stage of visual selection that "weights" stimuli according to their positions in space (e.g., Bundesen, 1990). The effects of different variables have then been defined in terms of whether they affect the limited selection process in the patients. For example, there have been several demonstrations that extinction can be reduced when stimuli in the contra- and ipsilesional fields group on the basis of local Gestalt properties such as collinearity, connectedness, and goodness of shape (e.g., Humphreys, 1998; Mattingley, Davis, \& Driver, 1997; Ward, Goodrich, \& Driver, 1994). These effects of Gestalt grouping can be contrasted with failures to find benefits from associative coding between objects (Humphreys, 1998; Riddoch, Humphreys, Edwards, Baker, \& Willson, 2003). From this it follows that Gestalt grouping cues may influence processing prior to visual selection, whilst higher level associative coding is influential at a postselection stage.

For the most part, additive factors logic, the psychological refractory period, and even neuropsychological procedures are applied independently of any constraints derived from neural-level investigations - indeed, a basic tenet of the emergent discipline of cognitive neuropsychology in the 1980s was that the nature and neural locus of the lesion was irrelevant for understanding the functional deficit suffered by the patient (Mehler, Morton, \& Jusczyk, 1984). This stance is encouraged by the view that different levels of theoretical description are quasi-independent. The problem here, though, is that these levels of description are ultimately not isolated from one another, and we need to find ways of crossing from one level to other, to use constraints at one level to inform theories at other levels.

\section{Interactivity Across Levels}

We can illustrate the importance of constraints from different levels of theory using, again, an example from studies of extinction. Although it has been demonstrated that patients with extinction can remain sensitive to the properties of stimuli in the extinguished field (Humphreys, 1998), it is not easy to deduce from behavioral data alone that these properties are processed normally. Patients are frequently slow to respond and make errors at stimulus exposures where controls remain at ceiling. To equate performance levels, to compare the relative magnitude of an effect, will often mean that there are major data reductions imposed on controls; the consequence is that the limitations on controls and patients may be different in kind.

This problem of assessing whether processing is normal or not can be helped by using converging evidence from a different level of analysis. For example, studies of functional brain imaging provide information about the activation of neurons in brain areas known to respond to low or higher level stimulus properties. By assessing whether activation in these low or higher level regions is normal, we gain new information that can complement the behavioral data. Of interest here are studies focusing on undamaged areas of the brain, where activation is not directly compromised by the lesion. Functional magnetic resonance imaging (fMRI) studies of extinction have shown that, although striate and extrastriate cortex in the lesioned hemisphere can be spared and are activated by stimuli that are extinguished, this activation is reduced relative to cases where single stimuli appear on the contralesional side (Ress, Backus, \& Heeger, 2000). Such data suggest that the PPC interacts with "earlier" cortical regions (striate and extrastriate cortex), with feedback from parietal cortex being important for the normal operation of those earlier regions. When a parietal lesion weakens feedback within one hemisphere, spatial biases in selection emerge. Note that a critical new piece of evidence is introduced here by brain imaging. There is a vast literature showing that striate and extrastriate ventral cortex respond to basic properties of visual stimuli and likely support the first stages of pattern and object recognition. Knowing this, we can then infer that the reduced selection capacity associated with parietal extinction emerges out of weakened modulation of earlier visual processing. Furthermore, if modulation is partial rather than being all-or-none, then it is possible for extinguished stimuli to enter into grouping and to activate higher level representations, but any such activation will be weaker than in the unlesioned state. Grouping effects may be present, but they are not necessarily normal in strength.

A further functional point brought out by this example is that processing appears not to operate in a completely feedforward fashion, because recurrent feedback from PPC seems necessary to balance competition across the fields. Apparently even the "simple" detection of a stimulus can depend on dynamic interactions between cortical regions. As well as promoting the idea of different levels of analysis, Marr (1982) was also influential in proposing a feedforward approach to information processing, emphasizing that the visual system should extract as much information as possible in a purely bottom-up manner. However, at a neural level, backward connections are at least as numerous as feedforward connections (Mumford, 1992), and, as the extinction example illustrates, there are good grounds for supposing that these feedback connections have a necessary function in cognition. To understand information processing, we need to understand the dynamic contributions from feedforward and feedback processes over time, and this involves more than a one-sweep feedforward computation.

\section{Computational Modeling in a Connectionist Framework}

One way to understand how interactions take place between different component processes is to build models of the components and to simulate the emergent whole-system behavior. Over the past twenty years or so, we have learned a good deal about how 
component processes may combine to generate whole-system responses through computational modeling (Bundesen, Habekost, \& Kyllingsbaek, 2005; Grossberg \& Grunewald, 1997; Léveillé, Versace, \& Grossberg, 2010). Particularly relevant here is the development of connectionist modeling as a way to capture the microgenesis of processing. Connectionist models have attempted to introduce some aspects of biological plausibility into cognitive psychology. The models use many units that compute relatively simple functions and communicate by passing activation between units. The influence of one unit on the other units to which it connects is modulated by the strength of the connection between the units, and the strength of this connection can be tuned by learning. Within such models both the temporal dynamics and the interactivity of information processing may be approximated more closely. Our concern is with modeling visual attention, where several connectionist models have been put forward (e.g., Pouget \& Bavelier, 2007; Tsotsos, Culhane, Wai, Davis, \& Nuflo, 1995). In one example, the selective attention for identification model (SAIM; Heinke \& Humphreys, 2003), feedback from stored knowledge units is used to modulate the competition between visual inputs to be represented in a focus of attention. Presenting multiple objects in the field increases the competition and slows the rate at which a target enters into a focus of attention, simulating the lengthening of reaction times (RTs) in multiple-object search tasks (Heinke, Humphreys, \& Tweed, 2006). Feedback from the knowledge units can bias attention to spread across features that together activate a common stored representation, and this topdown activity can override spatial reductions in input, as might occur in patients with visual extinction, so that effects of grouping may emerge despite the spatial reductions. Interestingly, grouping in this model is a product of top-down feedback, contrary to the inference that grouping in extinction reflects processes operating in a bottom-up manner.

However, as far as providing a link to real neuronal processes is concerned, these models leave much to be desired. Due to the lack of an explicit link between connectionist models and the underlying neurophysiological substrate, the dynamical evolution of the changes in network states (e.g., from a level of spontaneous activity through to an emergent response to a stimulus) and the description of inherent network fluctuations are not necessarily captured in a way that reflects real neuronal behavior. An example of this is that temporal parameters in such models are typically divorced from the temporal properties of real neurons, making it difficult to predict how operations at the whole-system level of behavior may reflect dynamic changes at a neural level. Here we show that, by modeling at a neural level, a direct link can be forged based on the temporal parameters of neurons.

\section{Toward Modeling Real Neural Networks}

\section{Critical Properties of Real Neuronal Networks}

What are the properties of real neuronal networks that may be critical for developing models that link physiology to behavior? Indeed, is there a specific level of modeling that would work in this respect, or are we on a course for infinite regress, with the physiological level itself not being enough and with explanations needing to operate further down again, say at a molecular level? As we try to elaborate below, we are not arguing for infinite regress.
Rather we are arguing for there being a rational level of analysis based on the physiological operation of single neurons that can be generalized in a direct (mathematically tractable) manner into subnetworks of neurons, which can then be related to psychological functions. Moreover, in developing and applying the models that incorporate the critical physiological properties, we believe that important emergent issues are made explicit, which we attempt to illustrate through our modeling of human visual attention. Our work highlights three emergent issues related to (a) the timing of neuronal processes, (b) the nature of neural degeneration, and (c) the decomposition of neural networks revealed through neuralimaging data (e.g., fMRI). We also go on to outline new predictions for whole-system behavior that we test. We argue that, though these issues could be raised in higher level models, they are not brought to the fore in quite such a direct way. Importantly, the issues are not just relevant to data collected at the physiological level but also to data speaking to the component processes isolated by cognitive psychologists.

So, what might be an appropriate level of analysis to enable an explicit bridge to be built from physiology to behavior? We propose that, on the one hand, models need to take into account those neuronal and synaptic mechanisms that are well characterized by a large amount of physiological and anatomical data. On the other hand, the description should be simple enough to enable us to infer by abstraction the first principles or computational correlates of the brain function(s) under study. In this respect, it is not sufficient for simulations "merely" to reproduce psychological and neurophysiological phenomena (see the discussion in Heinke \& Mavritsaki, 2009). Simulation, without elucidation of a computational mechanism, will tell us little more than what we already know from observing highly complex biological systems in action-not to mention the fact that we are then set the problem of establishing free parameters in the absence of biological or computational constraints (see Heinke \& Mavritsaki, 2009). The main aim of computational neuroscience is, rather, to provide a theoretical framework for formulating explicitly our theoretical assumptions in the light of observed experimental constraints (at both physiological and psychological levels of analysis). In this sense, we are faced with an inverse problem: We have to extract the free parameters of a system that cannot be measured directly (e.g., the connectivity between the thousands of neurons making up any plausible subnetwork) but that can be inferred by (a) studying the dynamical capabilities of the system and (b) looking for regions within a parameter space that generate an emergent behavior consistent with the experimentally measured observations.

We assume that a proper level of description at the microscopic level is captured by the spiking and synaptic dynamics assuming one-compartment, point-like models, like integrate-and-firemodels. The operations in such models are based on plausible biophysical constants (for conductances, response latencies, etc.), which can then be used for studying the realistic time scales and firing rates involved in the evolution of the neural activity underlying cognition. The resulting functions can be contrasted with experimental data. We believe that this is essential for several reasons:

1. The brain is a dynamical system that is exquisitely sensitive to the different underlying spiking and synaptic latencies. For this reason, it is convenient to include a thorough description of the different latencies for synaptic activity by implementing models 
with plausible properties for synaptic receptors (e.g., the fast and slow excitatory receptors [AMPA and NMDA] and GABAinhibitory receptors).

2. A temporally realistic, detailed level of description of synaptic activity is required to capture data at different levels of description, including neuroimaging as well as behavioral data. For example, simulations of fMRI data should involve the realistic calculation of blood-oxygen level-dependent (BOLD) signals that are intrinsically linked to synaptic dynamics (see Logothetis, 2008; Logothetis, Pauls, Augath, Trinath, \& Oeltermann, 2001).

3. In manipulating integrate-and-fire neurons we can consider the influence of neurotransmitters and pharmacological manipulations (e.g., the influence of dopamine on NMDA and GABA receptor dynamics; Durstewitz, Seamans, \& Sejnowski, 2000; Zheng, Zhang, Bunney, \& Shi, 1999), to study the effect on both global network dynamics and the related cortical functions (e.g., working memory; Brunel \& Wang, 2001).

Now, because models at this level explicitly incorporate different neurotransmitter functions, because they show specific time courses of neuronal firing (and delays in responding) using time constants taken from real neurons, and because they naturally incorporate neuronal noise, then the models begin to show how these issues are critical for understanding cognition. The models also provide a pathway for going from single-cell to whole-system behavior, which we illustrate.

To make concrete our arguments, we draw on studies into the modeling of visual attention we have conducted using neural-level models that exploit the idea that attentional selection operates through biased competition (Duncan et al., 1997). When multiple stimuli are presented, the items compete to control behavior. Which stimuli are "selected" to control behavior is determined by both bottom-up factors (e.g., the local contrast between an item and its surround) and top-down factors (e.g., the goals of the task), which bias the competition between the items. These bottom-up and top-down biases are captured in neural-level models that can simulate results ranging from single-cell recordings through to measures of human visual search. In Studies 1 and 2 we introduce the models and show how single-cell and whole-system behavior can be captured. In Studies 3 and 4 we present modeling results on the effects of brain lesion and on simulating neuroimaging data, which capture emergent results brought about by neural-level modeling. In each of these studies, we show how the modeling work gives rise to new qualitative predictions about how human selection should vary as a critical parameter of the model is varied. Importantly, the parameters in question reflect the adoption of biologically plausible assumptions at a spiking level. In Study 5 we put these predictions to empirical test. The results show how modeling can play a key role at the center of cognitive neuroscience, directing new research, simulating existing results, and integrating findings across different levels.

\section{Spiking Models}

Spiking-level neurons are complex and computationally expensive to implement. One way, then, to explore the implications of processes operating at the spiking level is to incorporate multiple neurons into one module within a larger computational system that operates using a different principle (e.g., using a higher level connectionist format) and to examine how the operation of the spiking units impacts on the whole. An example of this comes from the work of Usher and colleagues (Usher \& Davelaar, 2002; Usher \& McClelland, 2001). These investigators were specifically interested in the role of the subcortical nucleus, the locus coeruleus (LC), in regulating human attention and memory (see also Nieuwenhuis, Gilzenrat, Holmes, \& Cohen, 2005). The LC is known to exhibit specific patterns of response in relation to the occurrence of target stimuli, with a stimulus-locked phasic response being distinguished from tonic (nonstimulus locked) activity. This activity can also be linked to the release of the neurotransmitter norepinephrine, which in turn regulates higher level cortical responses involved in decision making. Usher, Cohen, Servan-Schreiber, Rajkowski, and Aston-Jones (1999) first modeled the operation of the LC in terms of a reduced version of a nonlinear spiking system with two variables ${ }^{1}$ that captured the fast phasic and slow tonic modes of operation. Activity from their LC was fed into a relatively simple connectionist model, whose job was to make either a go or a no-go decision to a given input. Both the connectionist part of the system and the LC part received perceptual input and fed activity into the perceptual and response layers of the connectionist model, with output from the LC part altering the gain of the activation function within the connectionist model. These simulations show that it is possible to integrate a relatively simple spiking-level model, with its accompanying dynamic characteristics, with a connectionist model, and the resulting simulations may enable us to understand better variations in detection and memory over time in direct relation to changes in LC activity.

However, there are limitations to such hybrid models. Most notably, it is not possible to take the system as a whole and to map it onto physiological data, as the connectionist part of the model does not mimic the activation functions found in real neurons. In some sense, the model provides an existence proof that the spiking-level module can have the demonstrated functional effect on another system making detection and recognition responses, but it does not show that the detection and recognition processes themselves operate in this way in a real biological system. We cannot make an inference from the model back to neurophysiologyand this is one of the things that any bridge between neurophysiology and behavior must do. It must allow physiological data to be rolled out through a model in order to generate predictions for wholesystem behavior, and it must allow inferences to be made from models of psychological data to help predict and understand neurophysiological data. To make such an inference we need to use models that integrate neurophysiological constraints throughout the model.

\section{Neuronal-Level Model}

The functional specialization of the brain emerges from the collective network dynamics of cortical circuits of coupled spiking neurons. Each neuron transforms a large set of inputs, received from different neurons, into an output spike train, that constitutes the output signal of the neuron. This means that the spatiotemporal

\footnotetext{
${ }^{1}$ Specifically, they used the Wilson-Cowan (Wilson \& Cowan, 1972; excitatory-inhibitory) population system, which can be thought of as an approximation of real neuronal operation (Gilzenrat, Holmes, Rajkowski, Aston-Jones, \& Cohen, 2002).
} 
spike patterns produced by neural circuits convey information between neurons; this is the microscopic level on which the brain's representations and computations rest. A simplified version of a model that can include as many properties as possible of the microscopic level is the integrate-and-fire neuron. Integrate-andfire neurons can incorporate important neurotransmitter properties that provide a bridge between physiology and behavior. Below, we present studies where spiking-level neurons were used. The equations for the spiking level of these studies are presented in Figure 1. These equations describe the subthreshold membrane potential of the neuron, as they are dependent on the synaptic currents that enter the cell (Deco \& Rolls, 2005a). In the central nervous system, the excitatory synapses rely on glutamate as their neurotransmitter, with different types of glutamate receptor present in each synapse. The channels that are controlled by AMPA receptors are described by a fast response to a presynaptic current and quick decay in the postsynaptic current; in contrast, channels that are controlled by
NMDA receptors are described by slow dynamics. NMDA and AMPA equivalents are present in our integrate-and-fire model description in Figure 1. In addition, fast inhibitory neurons present in our central nervous system are mainly regulated by $\gamma$-aminobutyric acid (or GABA); a description of the GABA currents is presented in Figure 1. Additionally, the neurons in the model include a frequency adaptation current, which also characterizes real neuronal operations (Liu \& Wang, 2001). Animal studies demonstrate that the $I_{A H P}$ current is primarily responsible for frequency adaptation over the first $300 \mathrm{~ms}$ of stimulus processing (Madison \& Nicoll, 1984). The mechanism of the $I_{A H P}$ current can be described as an influx of a small amount of calcium $\left[\mathrm{Ca}^{2+}\right]$ every time an action potential is generated. The influx of $\left[\mathrm{Ca}^{2+}\right]$ increases the intracellular levels of $\left[\mathrm{Ca}^{2+}\right]$ concentration, which in turn activates the $I_{A H P}$ current that inhibits the activation of the cell (Liu \& Wang, 2001). The system also has external neurons that are modeled with AMPA-like dynamics. More details for the neuronal

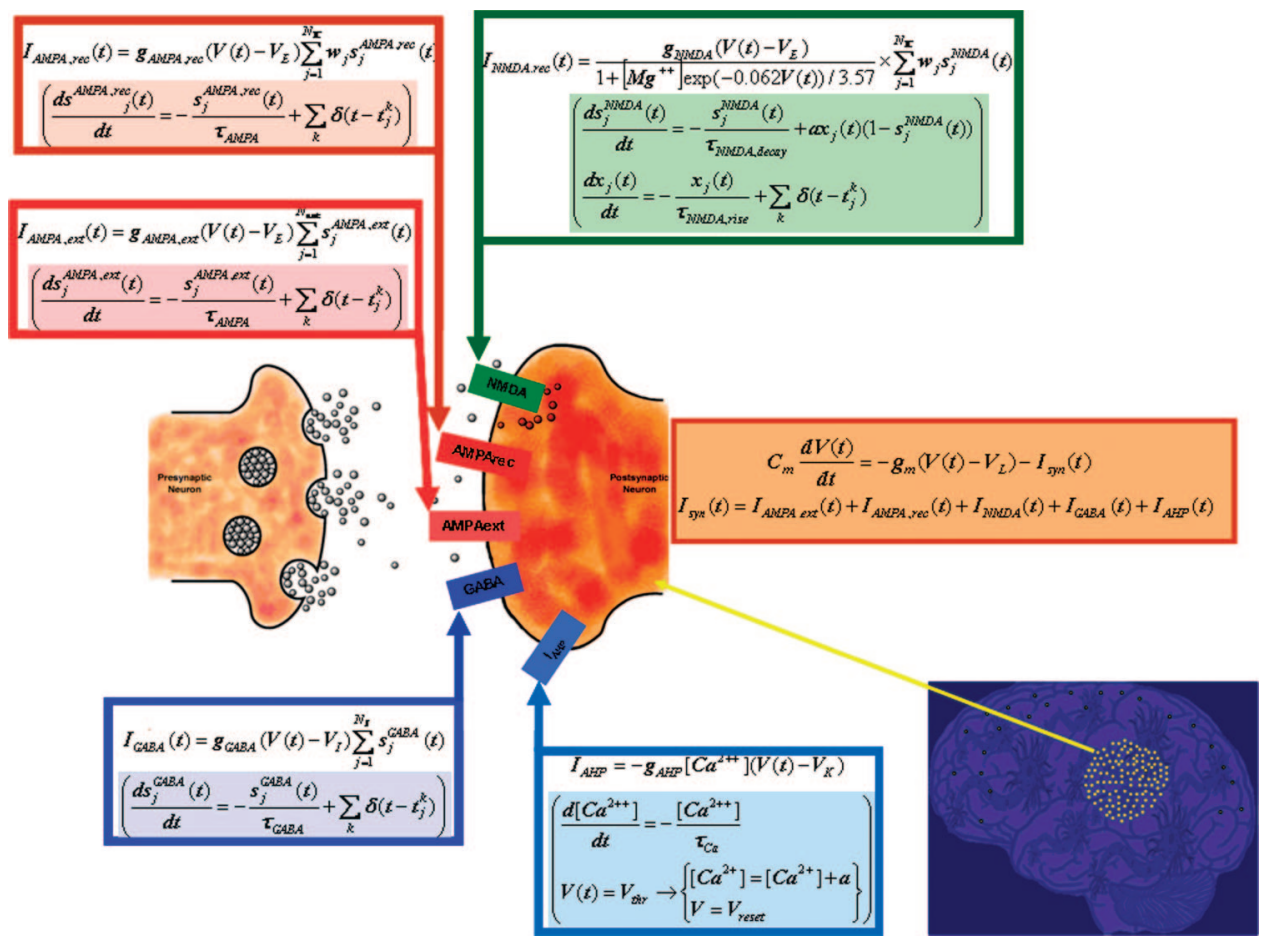

Figure 1. The subthreshold membrane potential of the cell can be described by the equation shown next to the postsynaptic cell. $C_{m}$ is the membrane capacitance where different values are given for excitatory $C_{m} e x$ and inhibitory $C_{m}$ in neurons; $g_{m}$ is the membrane leak conductance where different values are also given for excitatory $g_{m} e x$ and inhibitory $g_{m} i n$ neurons; $V_{L}$ is the resting potential; $I_{s y n}$ is the synaptic current; and $I_{A H P}$ is the current term for the frequency adaptation mechanism. The synaptic currents are described in the corresponding boxes; in each box the portion of open channels is highlighted. $V_{E}$ is the excitatory reversal potential; $w_{j}$ are the synaptic weights; $g_{A M P A, r e c}$ is the synaptic conductance; and $s_{j}^{A M P A, r e c}$ is the proportion of open channels for AMPA,rec. The voltage of the NMDA recurrent currents $I_{N M D A, r e c}$ is determined by the extracellular magnesium $\left[\mathrm{Mg}^{2+}\right]$ concentration (Jahr \& Stevens, 1990), where $\left[\mathrm{Mg}^{2+}\right]$ is the concentration of magnesium, $g_{N M D A}$ is the synaptic conductance, and $s_{j}^{N M D A}$ is the proportion of open channels for $N M D A$. For the inhibitory GABA currents $I_{G A B A}, g_{G A B A}$ is the synaptic conductance and $s_{j}^{G A B A}$ is the proportion of open channels for this receptor type. The connections with the external neurons follow AMPA-like dynamics and the voltage $I_{A M P A, e x t}$, where $g_{A M P A, e x t}$ is the synaptic conductance and $s_{j=1}^{A M P A, e x t}$ is the fraction of open channels. $V_{K}$ is the reversal potential of the $K^{+} ; g_{A H P}$ is the synaptic conductance; $\alpha$ is the $\left[\mathrm{Ca}^{2+}\right]$ influx; and $\tau_{\mathrm{Ca}}$ is the leaky integrator's decay constant. [ $\left.\mathrm{Ca}^{2+}\right]$ concentration is initially set to 0 , and the values can be found in Deco and Rolls (2005a). The parameters for the equations are given in Appendix C. 
properties can be found in Mavritsaki, Heinke, Humphreys, and Deco (2006); Brunel and Wang (2001); and Deco and Rolls (2005a).

\section{Mean Field Approximation}

Our argument is that this neuronal description allows us to build a bridge between behavior and physiology. However, we are nevertheless faced with severe problems in scaling up the model, to capture a level of subcomponent operation that relates to psychological studies, given that any such model will involve multiple nonlinear dynamic equations whose operation is computationally intensive and difficult to simulate. This is where the mean field approach comes in. In this approach, individual integrate-and-fire neurons are grouped together into populations of statistically similar neurons. A statistical description of each population is given by a probability density function that expresses the distribution of neuronal states (i.e., the membrane potentials) across the population. The key assumption in the population density approach is that the afferent input currents impinging on neurons in one population are uncorrelated and, consequently, the dynamics of the model can be described by the evolution of the probability density function. The mean field approximation entails replacing the time-averaged discharge rate of individual cells with a common time-dependent population activity (the ensemble average) where, in a statistical sense, the rate of a single neuron calculated across time is equivalent to the rate of activity across the neurons in a given population at a given time (see Figure 2). The whole-system behavior that we may witness in many psychological experiments represents what we may term the steady state of the underlying neurodynamical system (following its transient evolution to this state). To get to this steady state for a model, and to capture the transient changes of the network en route, we need to find the appropriate parameters for the model (in general terms, the connectivity parameters for the network). This is an inverse problem-we have to go from the whole-system behavior (the behavioral steady state) to the parameters that generated this in the first place. There are several problems with this, though. One is practical-it would take an enormous amount of time to explore the parameters that would make a given model approximate a network in the brain. A second, deeper issue is that the results of these simulations are probabilistic (i.e., there will be a certain probability that the neurons in a population are in a given state). This makes it particularly difficult to explore the parameter space in a systematic fashion, because any set of parameters must describe a probability distribution and not a single point in the parameter space. To overcome this problem, the mean field approach is applied when a model reaches a stable

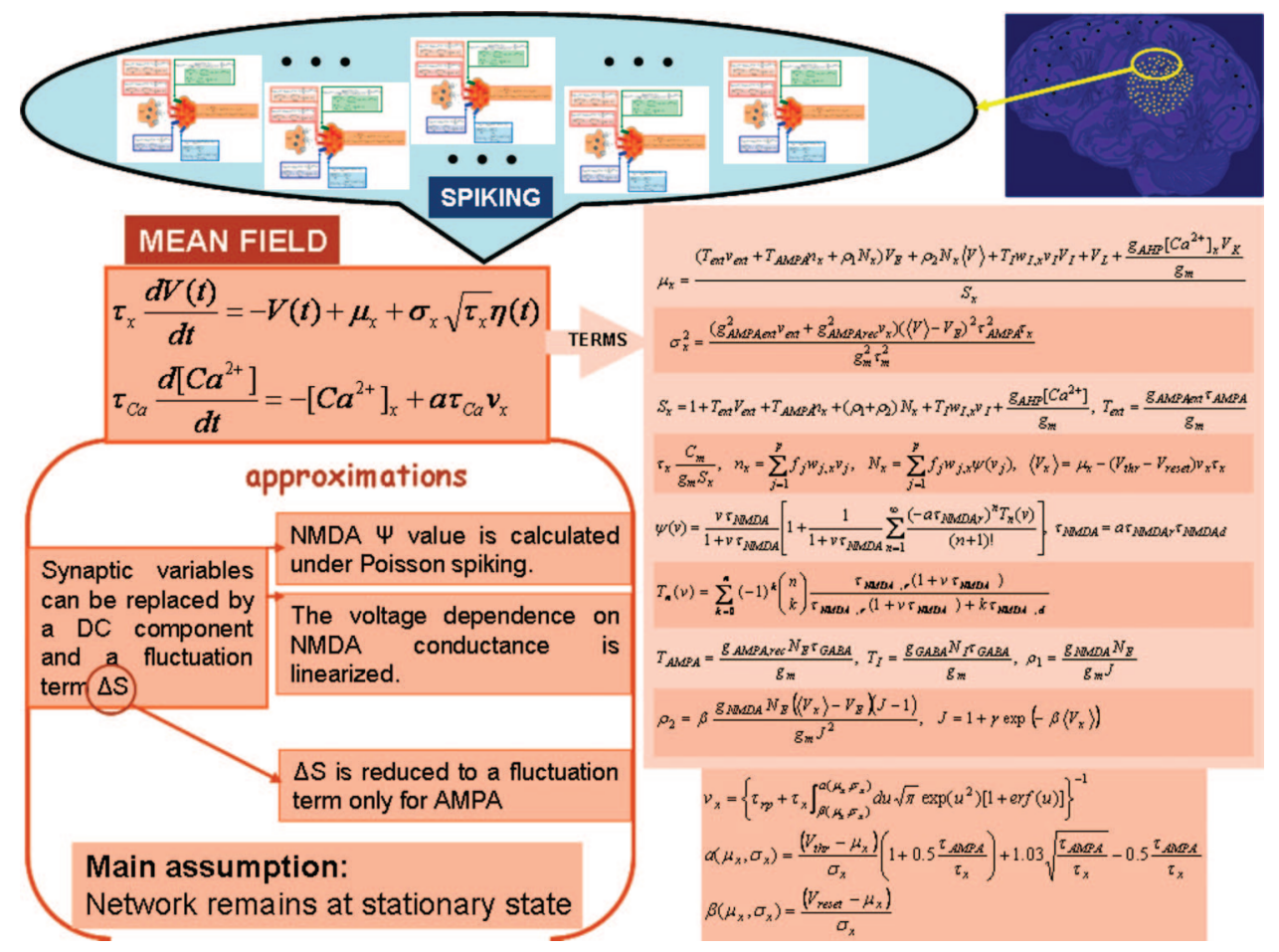

Figure 2. In the mean field approximation, the equations that represent a group of neurons in the brain (see top of the figure [blue]) are approximated with only one equation, based on the assumption that the system remains in a stationary state. The approximations that are implemented are highlighted in the box below the mean field equation. $\tau_{x}$ is the effective membrane time constant, $\mu_{x}$ is the asymptotic value the membrane potential would have in the absence of fluctuations and in the absence of spiking, and $\sigma_{x}$ measures the magnitude of the fluctuations in the synaptic inputs. $\eta(t)$ is a Gaussian process with an exponentially decaying correlation function with time constant $\tau_{A M P A}$. For the $I_{A H P}$ current an adiabatic approximation is assumed. The terms of the mean field equation are described in the box appearing on the right. 
(attractor) state after receiving a given input. When in a steady state, a population transfer function can be applied that describes the average spiking rate at a given time across the population (the population rate) as a function of the average input current. The steady state of the network, solved through the mean field approach, can be matched to a behavioral steady state, observed in a given experiment. In finding the parameters that generate this behavioral steady state, we solve the inverse problem. A critical point here is that, in the mean field approximation, all the biophysical details contained in the original neural-level model are maintained because the function is derived consistently from the original spiking equations. This is not the case for connectionist models because the activation functions for these models are essentially arbitrary with regards to the biophysical substrate.

There is an additional point. The solution derived at the mean field level holds only for the steady state of the network. It does not describe the transient states that the network goes through to reach the steady state. The problem here is that some of the theoretically most informative aspects of experiments reflect the transient states that are passed through to obtain any final steady state. For example, at the mean field level the parameters for a model of visual selection may be set so that the target is consistently found (the attractor state we want to reach). However, mean field-level simulations do not give us information about how much time we need to converge to that attractor, and yet the time to find the target is the essence of tasks such as visual search, where selection is measured. Within our framework, we can solve this problem by applying the parameters found at the mean field level to spikinglevel simulations, precisely because these parameters have been derived consistently from the integrate-and-fire level. These spiking-level simulations then give us the transient changes that we are interested in.

Following this approach, the aim is to derive parameters at the mean field level in order to simulate a key behavior that we are interested in (e.g., successful visual search that approximates the rate of human search in a critical condition). Following this, a spiking-level variant of the model can be developed to capture the dynamic aspects of performance-for example, the time course of human performance as the temporal relations between search displays are varied. The additional parameters implemented at the spiking level, reflecting, for example, synaptic transmission processes, then need to be biologically plausible, so that direct links can be made between physiology and higher level behavior. We contend that it is important to have a direct "vertical" transfer of parameters from the spiking level to the mean field level, in order that we have a transparent means of linking the different levels of description. This is critical for making an inverse inference concerning how the real neural system must operate, based on the parameters initially derived to capture behavioral data in the model at the mean field level-one part of the two-way bridge between physiology and behavior.

In order to process from the spiking level to the mean field, a number of approximations need to take place, which are highlighted in Figure 2 and presented in more detail in Brunel and Wang (2001) and Deco and Rolls (2005a). Critical here is the diffusion approximation, where the synaptic variables for the different currents presented in Figure 1 are represented by an average DC component and a noise term. The noise term of all the synaptic currents can be approximated by the noise term of the AMPA, ext current, because the amplitudes of fluctuations of the AMPA currents are much larger than those of the NMDA and GABA currents. Two other important approximations concern the NMDA current: (a) The function $\psi(v)$ (i.e., the nonlinear dependence of the NMDA component on the pyramidal cell discharge rate) can be calculated if we consider it in terms of a Poisson distribution, and (b) the voltage dependence of the NMDA conductance is linearized under the assumption that the voltage stays in an area around the mean.

\section{Study 1: Modeling at a Neuronal Level (Deco \& Rolls, 2005a)}

Deco and Rolls (2005a) presented a skeleton model of biased competition that illustrates critical properties of the extended model (the spiking selection over time and space [sSoTS] model) that we elaborate on below. Deco and Rolls's work also illustrates how neural-level models can be used to capture effects from single-cell electrophysiological recording, whilst being extendable to model whole-system behaviors, as we illustrate. The framework for their model is set out in Figure 3. Unlike for our presentation of the sSoTS model (Studies 2-5), we do not discuss the parameterization of Deco and Rolls's, and we refer the reader to the original article.

The Deco and Rolls (2005a) model contained a simplified network with two layers, one of which may be thought to have processing units with characteristics of neurons in area V2, the other containing processing units with characteristics of neurons in area V4. Within each layer, there are excitatory neurons (a, b, c, d) that respond preferentially to particular stimuli (e.g., a particular orientation or a particular spatial frequency, in V2). The specific excitatory neurons in $\mathrm{V} 2(\mathrm{a}, \mathrm{b})$ are held to respond to local elements within their receptive field. The specific excitatory neurons in V4 (c, d) are held to have larger receptive fields than the neurons in V2 so that both sets of excitatory neurons within V4 respond to both sets of local excitatory neurons in V2. The V4 neurons were also set to have a "preferred" stimulus within V2 (indicated by the thicker connections from the V2 neurons to the V4 neurons, indicating larger connection weights; $\mathrm{a} \rightarrow \mathrm{c}$ and $\mathrm{b} \rightarrow$ d), whilst being activated to some degree by the other (nonpreferred) properties (the thinner connections from V2 to V4 indicating smaller connection weights; $\mathrm{a} \rightarrow \mathrm{d}, \mathrm{b} \rightarrow \mathrm{c}$ ). Simulations were conducted assuming that stimuli corresponding to oriented bars of particular spatial frequency were present, to activate the corresponding neurons in V2. When both sets of excitatory neurons in V2 were active, then both sets of V4 neurons would be activated too. The stimulus-specific excitatory neurons in each layer were also connected to two other sets of neurons: (a) a set of inhibitory interneurons (left of Figure 3) and (b) a set of nonspecific excitatory neurons assumed not to be connected to afferent input. The inhibitory neurons modulate competition between the excitatory neurons within each layer. The nonspecific excitatory neurons introduce noise into the operation of the model and help it to generate neuronally plausible spike-firing patterns. As we illustrate in Study 3, the presence of noise within neural-level models of this type turns out to have interesting consequences for modeling whole-system human behavior too. The excitatory neurons in the model were based on pyramidal cells and comprised $80 \%$ of the neurons in the system; the inhibitory neurons were modeled on 


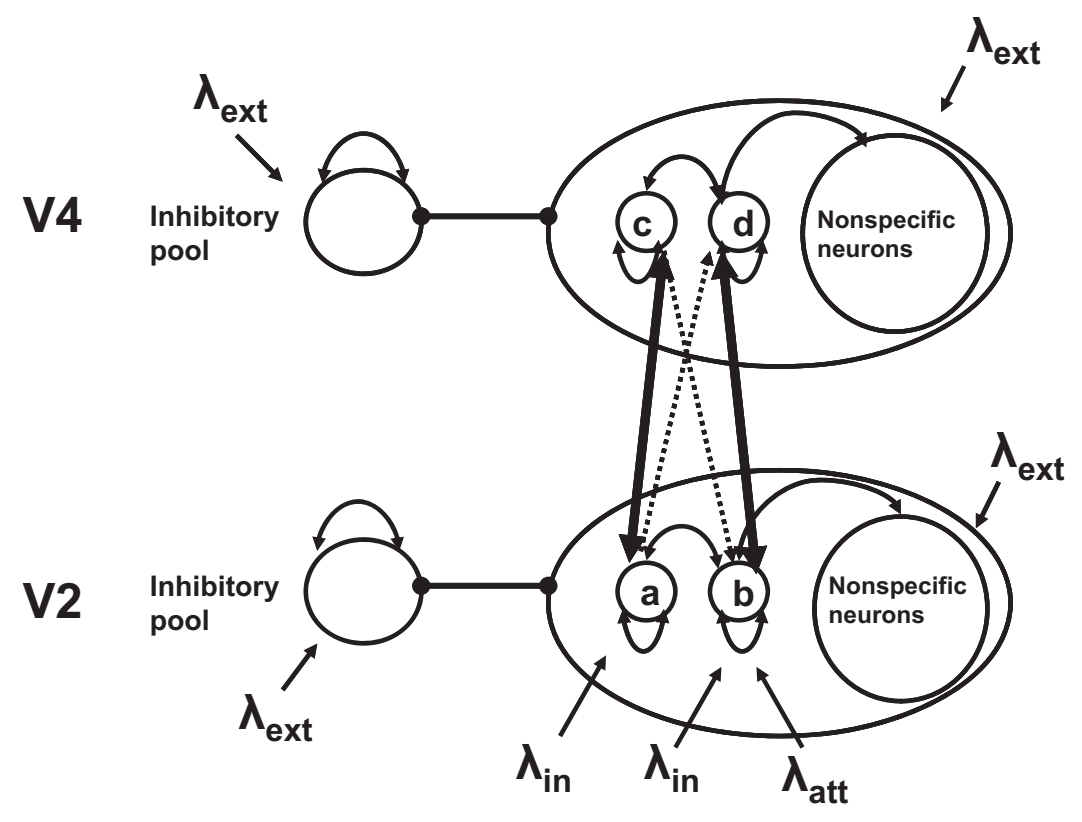

Figure 3. The model of Deco and Rolls (2005a). $\lambda_{\text {ext }}$ provides an externally driven level of spontaneous activity. $\lambda_{\text {in }}$ provides input into the system (from the visual field). $\lambda_{\text {att }}$ provides an attentional signal to a particular location. The receptive fields of neurons in level V4 are larger than those of neurons in V2, and so each covers more than one neuron within V2 (e.g., Unit c receives input from both Units a and b). Each cortical layer contains excitatory pyramidal neurons and inhibitory interneurons. Neurons are fully connected within each cortical layer (see Appendix A for further details). Neurons in each cortical level cluster into excitatory and inhibitory pools. There are two types of excitatory pool. Selective pools respond to specific visual features represented in connected cortical regions (e.g., a visual feature at a given location). The nonspecific cells do not have specific sensory inputs, but they have spontaneous firing and introduce noise into the system to produce Poisson-like spike firing (see Brunel \& Wang, 2001).

interneurons and comprised $20 \%$ of the neurons in the system. These proportions capture the approximate ratio of excitatory to inhibitory neurons in the cortex (Braitenberg \& Schüz, 1991; Brunel \& Wang, 2001; Deco \& Rolls, 2005a). All neurons in the model receive inputs from three currents: a fast inhibitory current mediated by GABA-like dynamics, a fast excitatory current mediated by AMPA-like dynamics, and a slow inhibitory current mediated by NMDA-like dynamics (see Figure 1). All of the neurons received some external input to provide a background level of firing $\left(\lambda_{\text {ext }}\right)$. The excitatory neurons in V2 also received specific afferent input, representing the presence of stimuli in the visual field $\left(\lambda_{\text {in }}\right)$.

This model was applied to simulate data from Reynolds, Chelazzi, and Desimone (1999; see also Moran \& Desimone, 1985). Reynolds et al. used a paradigm in which monkeys directed attention to a target stimulus across multiple trials. Differences between neuronal firing rates for attended and nonattended stimuli were seen relatively early in the time course, following presentation of the critical target stimulus. This does not, of course, rule out attentional modulation later in the time course (after, for example, texture segmentation tasks; Roelfsema, Tolboom, \& Khayat, 2007). Here, however, we concentrate on the case where the stimuli are clearly segmented from the background and discriminated from each other and participants receive information on some trials that allows them to purposely attend to specific stimuli.
In Reynolds et al.'s (1999) experiment, oriented bars were presented in the receptive fields of neurons in V2 and V4 of a monkey; one of the bars was the preferred stimulus for the V4 units being studied; the other was a nonpreferred stimulus. When the monkey was trained to attend to a location outside the receptive field, the presence of the nonpreferred stimulus in the receptive field reduced the neuron's response to the preferred stimulus, with the neuron responding according to the weighted average of the signals. When the monkey was trained to attend to the location of the preferred stimulus, the V4 neuron increased its firing. When the animal attended to the location of the nonpreferred stimulus, neuronal firing was suppressed. The effect of attention was modeled by introducing an extra attention input $\left(\lambda_{\text {att }}\right)$ to neurons in V2 with a receptive field at the attended location, when both a preferred and a nonpreferred stimulus were present in the receptive field of the neurons in V4. Deco and Rolls (2005a) examined the activity of sets of neurons in the specific excitatory pool in V4, and the results are shown in Figure 4. The pair condition corresponds to the case where the animal is assumed to be attending outside the receptive field, when the activity of the V4 neurons corresponds to the weighted average of responses to the preferred and nonpreferred stimuli when presented alone. In the pair + attend good condition, the attentional input is given to the location of the preferred stimulus for the neuron, whereas in the pair + attend bad condition, attentional input is given to the location of the neuron's 


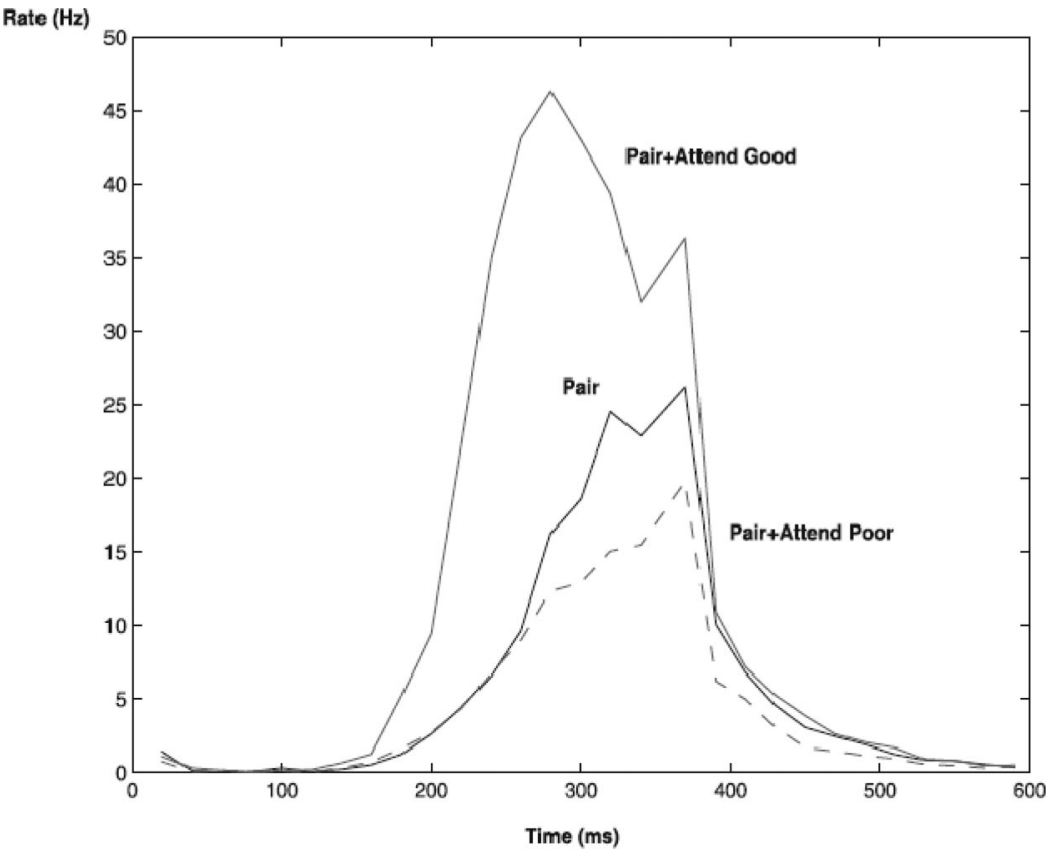

Figure 4. Dynamical activity in the Deco and Rolls (2005a) model at level V4. The figure shows the firing rate of V4 neurons to a good (preferred) or poor (nonpreferred) stimulus when both stimuli are present simultaneously in the receptive field. In the pair condition, there is no specific attentional input to the location of either the good or the poor stimulus. In the pair + attend good condition, attention is allocated to the location of the good stimulus. In the pair + attend poor condition, attention is allocated to the position of the poor stimulus. The stimuli were presented in the period of $100-350 \mathrm{~ms}$.

nonpreferred stimulus. As can be seen, the model showed clear modulation of neuronal firing, determined by whether the location of the preferred or the nonpreferred stimulus was attended. Relative to the baseline (no-attention, pair condition), there was enhanced activation when the location of the preferred stimulus was attended, whereas activity was suppressed when the nonpreferred stimulus was attended.

Deco and Rolls (2005a) also went on to show that these attentional modulations were influenced by the relative contrast of the stimuli. The effects of a nonpreferred stimulus were stronger when it had a higher contrast relative to the preferred stimulus, but attention to the preferred stimulus reduced this effect of salienceparticularly when the preferred stimulus itself was in an intermediate contrast range. This result mimics single-cell data reported by Martínez-Trujillo and Treue (2002) and Reynolds and Desimone (2003).

Prediction 1. Interestingly, the effects of attentional modulation in this model were influenced by the relatively long time course of the NMDA current (see Figure 1). When the time course of this current was reduced, the effects of attentional modulation were much weaker (Deco \& Rolls, 2005b). This is of interest for studies operating at higher levels of analysis because changes in the NMDA current may be thought to approximate the effects of arousal by altering global activation levels within the neural system. Mavritsaki, Heinke, Deco, and Humphreys (2009) altered the NMDA in an extended version of the Deco and Rolls (2005a) model (see Study 3) to simulate effects of reduced arousal associated with damage to right frontoparietal regions in neurological patients (see Robertson \& Manley, 1999). Mavritsaki et al. (2009) found that there was reduced selectivity in their model when NMDA modulation was reduced, which, combined with a spatial bias introduced into selection by lesioning their model, led to particularly poor selection of stimuli on the "bad" side of space. The NMDA effects also reduced selectivity on the "good" side, however, which fits with right frontoparietal patients having nonspatial deficits that are modulated by increasing arousal (see Malhotra, Parton, Greenwood, \& Husain, 2005; Robertson, Mattingley, Rorden, \& Driver, 1998). If alterations in NMDA function can be taken as an approximate means of varying physiological arousal (Mavritsaki et al., 2009), and if impaired arousal is associated with right frontoparietal damage, then a qualitative prediction follows There should be reduced attentional modulation of selection in patients with right frontoparietal damage, which should be related to impairments in arousal and not just to, for example, spatial biases in selection. This was tested in Study 5A here.

\section{Study 2: Modeling Whole-System Behavior in Search (the sSoTS Model)}

\section{The sSoTS Model}

The Deco and Rolls (2005a) model illustrates how spiking activity in a neural-level simulation can be directly related to single-cell activity. However as an account of human visual attention their framework is limited. For example, attention within the model acts by modulating activity at an attended location. How- 
ever, there is considerable evidence that participants can attend to and/or selectively ignore either the dimension that defines a target stimulus (Found \& Muller, 1996; Weidner \& Muller, 2009) or the value of the target feature (Braithwaite \& Humphreys, 2003; Folk, Remington, \& Johnston, 1992). There is no mechanism for nonspatial biasing of selection within the model. In addition, the model represents only two spatial positions, and it is not clear how well any competition for selection will scale when more locations are represented. We also do not know how activation emerging within a layer (e.g., in V4) can be used to "drive" other processes (e.g., an eye movement to the location of a stimulus that is attended) that may map on more directly to higher level behavioral studies; note that the receptive fields in V4 cover more than one location at the level of V2 in the model. Deco and Zihl (2001) developed an extended model that included feature maps that represent multiple spatial locations and that feed the outputs of competitive processing at the feature-map level into a location map, which effectively represents the saliency of activity at each location (cf. Itti \& Koch, 2001). However, this work used a high-level (mean field) approximation that did not include the full neuronal dynamics. A neural-level version of this extended framework, which we term the spiking search over time and space model (sSoTS), was put forward by Mavritsaki et al. (2006), and this forms the basis of the remaining studies we present here.

The architecture for the sSoTS model is presented in Figure 5. The model takes the same basic form as the Deco and Rolls (2005a) model, with there being pools of specific excitatory neurons, nonspecific excitatory neurons, and inhibitory neurons. Unlike in Deco and Rolls (2005a) model, specific excitatory units representing different visual features are coded for different locations in distinct spatiotopic maps; this follows the common assumption that features are coded in distinct spatiotopic maps in early vision (Treisman \& Gelade, 1980). Within each feature dimension, though, there are common inhibitory units that constrain activation to favor only one feature occupying one spatial position during selection. There are maps for each of two features (e.g., blue and green) in each of two dimensions (color and shape), with the specific excitatory units in each map representing six spatial locations. In order to show how outputs from such units may be used to drive other behaviors, activation within the feature maps is fed through to a location map that sums activity across maps and passes activity back to units within the feature maps in order to sharpen the competition between the stimuli at that level. Activity in the location map reflects the relative salience of the different stimuli competing for selection, whilst also conveying information (for other systems, not in the model) about the location where a response may be targeted. The model uses similar processing dynamics to those presented in Deco and Rolls (2005a; Figure 1). For example, units in the feature maps receive input representing external stimuli being presented $\left(\lambda_{\text {in }}\right)$. All units also receive a background level of spontaneous activity $\left(\lambda_{\text {ext }}\right)$, with a Poisson noise distribution with value $3 \mathrm{~Hz}$, consistent with activity values observed in the cerebellar cortex (Rolls \& Treves, 1998; Wilson, O'Scalaidhe, \& Goldman-Rakic, 1994). There were, however, two exceptions. First, rather than attention necessarily being directed to the location of a stimulus, attention can be applied more flexibly. It can be allocated in terms of an excitatory input to a location (e.g., giving additional input into units at one position within the location map), but it can also be allocated to a dimension (e.g., activating all locations within the color maps) or to a feature (activating the blue map; $\lambda_{\text {excit }}$ ). Second, as well as using excitatory attentional input, we also used inhibitory attentional input $\left(\lambda_{\text {inhib }}\right)$ to optimize visual selection. We review the reasons for incorporating this suppressive component of attention below. The changes to the activation functions are presented in Appendix A.

The sSoTS model can be applied to tasks where participants are not set to attend to a particular location - an example would be visual search where participants typically know the features that define the target, but they have no foreknowledge of the locations of the features. Foreknowledge of a search target in sSoTS is provided by preactivating maps for the expected target features prior to the search items being presented. Following presentation

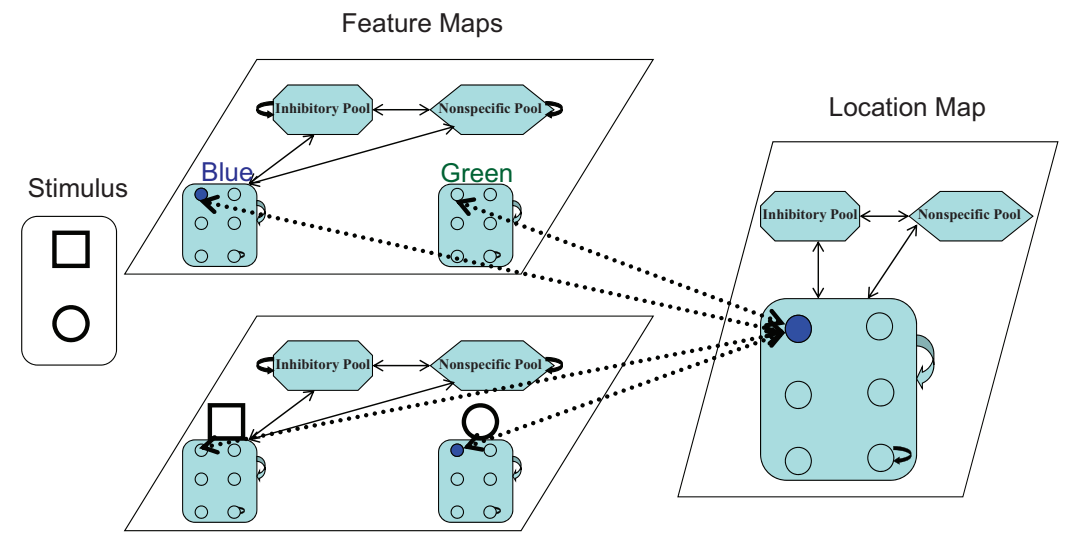

Figure 5. The architecture of the spiking selection over time and space (sSoTS) model. The model is composed of feature maps where units respond selectively to particular visual features (top map: color; bottom map: shape). Within each feature map, there are both selective and nonspecific excitatory neurons, with the selective neurons responding to particular features (e.g., blue or round). There is also a pool of inhibitory interneurons that are fully connected with the excitatory neurons. In the location map, the selective neurons respond to activity at corresponding locations in the field irrespective of the features present. 
of the search display there is competition for selection of the location (in the location map) where the target falls. Units within and across feature maps compete for selection through common sets of inhibitory neurons, but there is a net gain for target features from the top-down expectancy that is set. This leads to the target typically winning the competition for selection, with neurons at the target's position in the location map reaching a threshold for detection. The mechanism of setting an expectancy for the target's features is very similar to that employed in theories such as guided search (Wolfe, 1994), and, like guided search, the model predicts that differences should emerge for targets defined by a conjunction of features (where the target shares at least one feature with every distractor) and feature search (where the target differs from distractors through the presence of a critical single feature). In conjunction search, the top-down expectancy for the target's features will also preactivate feature maps responding to distractors, increasing competition. In single-feature search, the top-down expectancy uniquely activates the feature map for the target, so a greater competitive advantage for the target occurs. Activation profiles for different clusters of units in the location map are depicted for single-feature and conjunction search in Figures 6A and 6B. Mavritsaki et al. (2006) demonstrated that sSoTS generated the characteristic pattern of efficient and inefficient search (increasing nonlinearly or linearly as a function of the number of distractors) for conjunction and single-feature tasks, matching human data (see Figure 7). This result provides an existence proof that a model of this type, with parallel processing of visual elements that compete for selection, can generate linear search functions that have often been linked to serial models of visual selection (see also Heinke \& Humphreys, 2003; Townsend, 1972).

Preview search. In addition to being used to examine search across space, the sSoTS model has also been employed to study search over time. Human visual search for a conjunction target can be made more efficient if the temporal exposure of the distractors is staggered so that half the distractors appear as a preview before the other half (plus the target, when present; Watson \& Humphreys, 1997). Interestingly, this preview benefit for search has a long time course - search is optimal only when there is an interval of $500 \mathrm{~ms}$ or so between the preview and the new search display

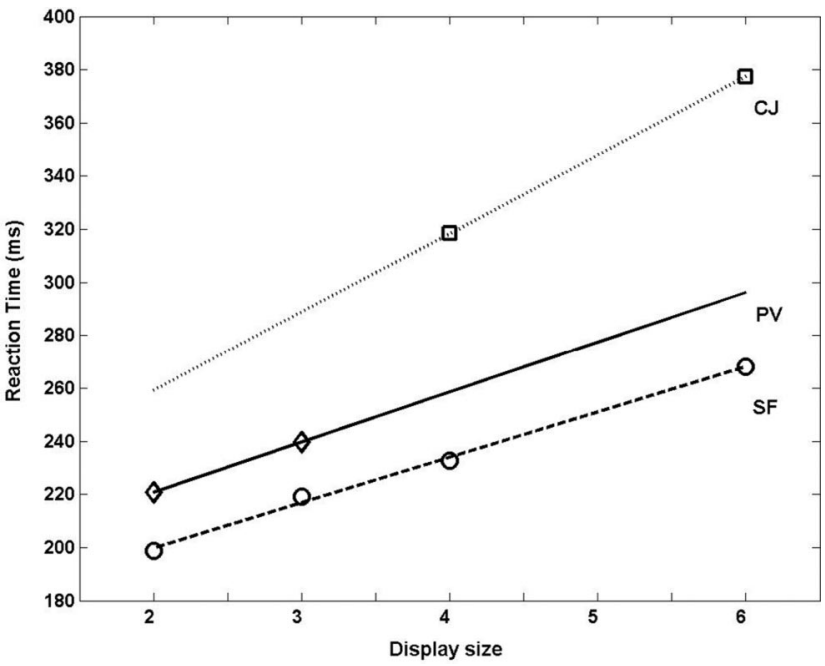

Figure 7. Time required for the spiking selection over time and space (sSoTS) model to detect the target in a search display. The data show the time for the winning set of units in the location map to reach threshold (see Appendix B for details). The data are shown here for conjunction (CJ), single-feature (SF), and preview (PV) search (with a preview duration of $1,000 \mathrm{~ms}$ ), and the search slopes are, respectively, 17.9, 29.53, and 18.89 ms/item. The reaction times (RTs) are derived from display sizes of 2, 3 , and 4 (single feature); 4 and 6 (conjunction); and previews with 2 and 3 items in both the preview and then a matching number of items in the new search display. Note that, in human RTs, any time taken to select the target location would be added to by motor response latencies.

(Humphreys, Jung Stalmann, \& Olivers, 2004; Humphreys, Olivers, \& Braithwaite, 2006; Watson \& Humphreys, 1997). Given that observers can temporally segment and group stimuli appearing within $100 \mathrm{~ms}$ of each other (Yantis \& Gibson, 1994), it is not clear why attentional benefits have such a long time lag.

Models that employ realistic biological parameters have the interesting property that neurons will adapt their firing over time. One mechanism of adaptation, incorporated into sSoTS, reflects a slow $\left[\mathrm{Ca}^{2+}\right]$-activated $\mathrm{K}^{+}$current (Madison \& Nicoll, 1984),
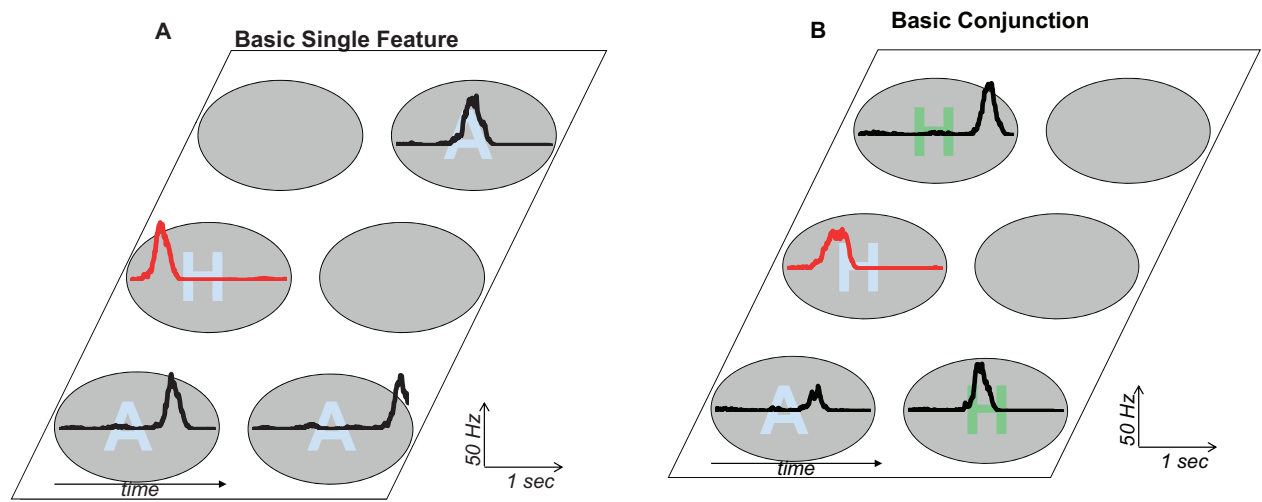

Figure 6. A: Activity in the neuron pools of the location map in the single-feature search condition. In each pool we have depicted the stimulus present at that location (in the external world). The plots show the activity over time. The target (blue $H$ ) falls at the center left location. B: Activity in the location map in the conjunction condition. 
which leads to decreases in firing rate after a cell has been active for a given time (the process of frequency adaptation; see also Deco \& Rolls, 2005a). Figure 8 gives the profile of $\left[\mathrm{Ca}^{2+}\right]$ adaptation across various parameter values. According to the parameters selected, there will be a buildup of $\left[\mathrm{Ca}^{2+}\right]$ across the first $300 \mathrm{~ms}$ or so of a stimulus, which results in decreased neuronal firing (see Figure 8). One consequence of this is that stimuli that have been presented for some time should decrease their activity, making them less salient for selection. The long time course of this process predicts that there should be a relatively sluggish process by which old stimuli lose their attentional interest over time, much as observed in the preview effect.

Parameter identification: From mean field to spiking neurons. To simulate data on preview search, the parameters of the sSoTS model were initially set at a mean field level (see above, Figure 2, and Brunel \& Wang, 2001; Deco \& Rolls, 2005a, for more details). The mean field simulations, which are much quicker than simulations at a spiking level, established areas in the parameter space where the activation was stable (stationary) and the target could be identified successfully in single-feature and conjunction search conditions as the main "within-map" properties were varied (e.g., inhibition within the location and feature maps; see Figure 9A for stationary activation and Figure 9B for successful target identification areas). These parameters were then incorporated into a spiking-level network, to identify the areas that simulate the correct slope and success rate for single-feature and conjunction search (see Figure 9C). The slopes and rates of successful target detections were calculated according to Appendix B.

Psychological research indicates that, in addition to any general effects of adaptation to old stimuli in preview search, there is also active suppression of distractors when participants prioritize search for the new stimuli. This evidence comes in various forms. Experiments have been conducted using probe procedures (where participants have to detect the presence of a probe on new or old

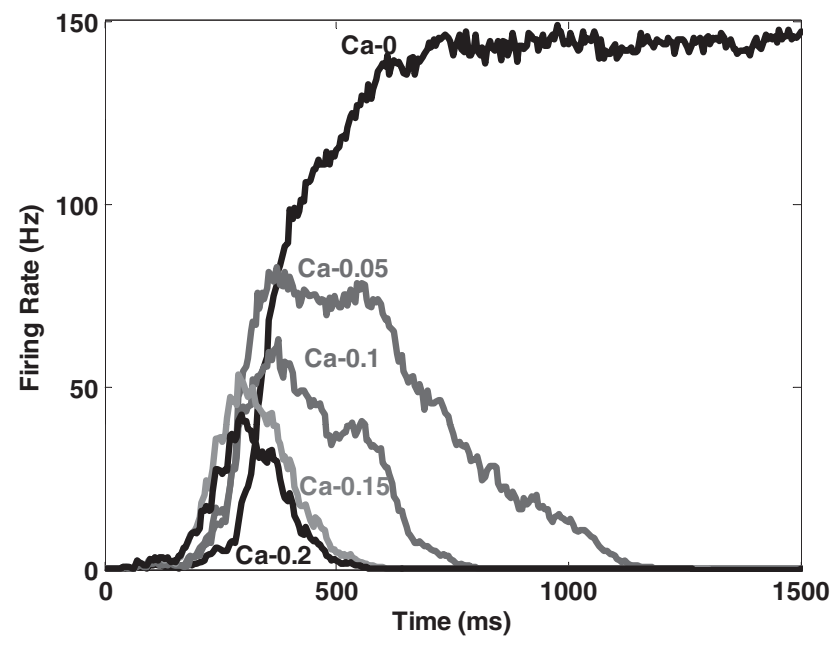

Figure 8. Illustration of variations in the neuronal firing rate as a function of the $\left[\mathrm{Ca}^{2+}\right]$ parameter. In each case when there is an increase in the concentration of $\left[\mathrm{Ca}^{2+}\right]$ entering the cell after the neuron spikes, there is increasing inhibition of the neuron and a decrease in the firing rate (frequency adaptation); altering the $\left[\mathrm{Ca}^{2+}\right]$ parameter changes the rate of this adaptation process. distractors or in the background) where it has been shown that probes on old objects are more difficult to detect than those in the background or on new distractors (Agter \& Donk, 2005; Braithwaite, Humphreys, \& Hulleman, 2005; Humphreys, Jung Stalmann, \& Olivers, 2004; Watson \& Humphreys, 2000). Such results are consistent with attentional suppression of old items that takes activation below baseline levels (probe on background). Other data indicate that this suppression can spread from the features of old items to new stimuli sharing the same features, making these new items difficult to detect (Braithwaite et al., 2005; Olivers \& Humphreys, 2003). There is also evidence for effects of old items appearing to have reduced contrast compared with new stimuli (Allen \& Humphreys, 2007). Importantly, this evidence for suppression of old distractors occurs only under conditions of attentional prioritization to new stimuli; there is little difference in the detection of probes on new and old stimuli when probe detection is the primary task (Humphreys, Jung Stalmann, \& Olivers, 2004; Watson \& Humphreys, 2000). Watson, Humphreys, and Olivers (2003) proposed that participants actively inhibit the old items to enhance any competitive advantage for new stimuli when they appear. To incorporate this form of top-down inhibition into sSoTS, we introduced a top-down suppressive as well as a topdown excitatory component into the model, with the inhibitory component $\left(\lambda_{\text {inhib }}\right)$ being added to units in the feature maps for old distractors.

Having initially set up model parameters to fit single-feature and conjunction search at the mean field level (see Figures 9A and 9B), the critical top-down excitation and inhibition parameters, and the $\left[\mathrm{Ca}^{2+}\right]$ parameter determining frequency adaptation, were set to match human performance in preview search in the spiking level.

The function for top-down inhibition is shown in Figure 10, which illustrates the effects of varying this parameter on the firing rate (left panel) and the attention modulation index for the location map (right panel). The net effect of the top-down inhibition is to initially raise activity in the feature maps corresponding to the location where the to-be-ignored feature is presented-this is because competition from units at other locations in the same feature map is damped down, so activity generated by the stimulus is enhanced. This enhancement, however, leads also to faster adaptation of the units, so that, at the level of the location map, activity from any initial, to-be-ignored distractors remains for a longer period if those distractors are not inhibited.

Explorations were conducted into the relations between the parameters determining active inhibition and those reflecting $\left[\mathrm{Ca}^{2+}\right]$ adaptation (see Figure 9D), to determine a region in the parameter space where preview search could be simulated (based on the slope of the search function under preview search conditions). The resultant RTs (directly based on the temporal firing patterns of the units) are presented in Table 1 for a "standard" preview condition (e.g., with the preview presented for 1,000 ms before the search display), along with RTs in the conjunction and feature search conditions. Activity profiles for units within the location map in preview search are presented in Figure 11. The slope of the search function was steeper in conjunction than preview search, whereas the slope for preview and single-feature search did not differ. This matches data on human search (e.g., Watson \& Humphreys, 1997). Search accuracy did not differ 

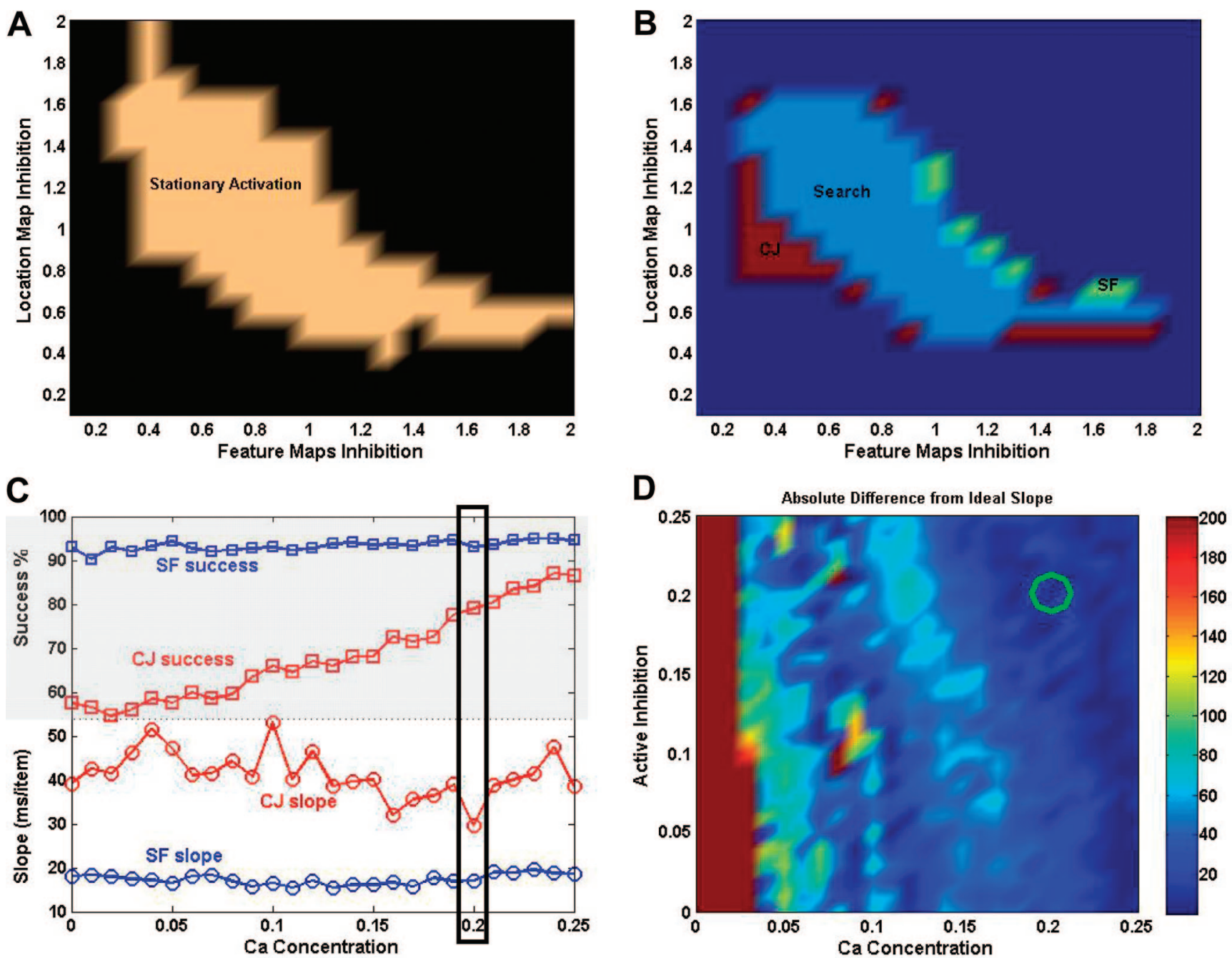

Figure 9. Exploration of the parameter space for the spiking selection over time and space (sSoTS) model. Panels A and B show the parameter set explorations at the mean field level. In Panel A, the yellow area shows the parameter pairs that generated stable (stationary) activity in the model, and the area in light blue in Panel B shows the parameter pairings that generated correct target identification for both single-feature (SF) and conjunction (CJ) search (in red are areas of the space where there was correct detection of the conjunction target only; in light green are areas of the space where there was correct detection of the single-feature target only). Panels C and D show the results from an exploration of the parameter space at the spiking level. The highlighted area was the region-selected value for the $\left[\mathrm{Ca}^{2+}\right]$ parameter. Panel D shows the difference relative to the "ideal" slope for preview search (taken from Watson \& Humphreys, 1997) as parameters for active inhibition and $\left[\mathrm{Ca}^{2+}\right]$ concentration were varied. The preview duration was set to $1,000 \mathrm{~ms}$.

across the conditions, and in all cases it was above $80 \%^{2}$ (i.e., the model selected the correct position in the location map as containing the target, when present).

These results indicate that sSoTS can generate the benefit for search over time (under preview condition) that matches human performance. Perhaps more critical, though, is that sSoTS also predicts a time course to the temporal segmentation benefit, based on the combined effects of top-down inhibition and $\left[\mathrm{Ca}^{2+}\right]$ adaptation. Taking the parameters set to give a preview benefit for a preview duration of $1,000 \mathrm{~ms}$, we assessed the time course of search in sSoTS as a function of different preview exposures. Note that the parameters were not changed further, and so they did not vary across the exposure durations. Human data reported originally by Humphreys, Jung Stalmann, and Olivers (2004) are shown in Figure 12. Figure 13 presents the results for sSoTS. Like human search, RTs for sSoTS are speeded as a function of the preview duration, and the difference between the display sizes (a measure of search efficiency) decreases up to a time when the preview reaches an optimum duration and then plateaus across a range of more extended durations. In sSoTS, suppression from active inhibition and from adaptation reduce the competitiveness of the old

\footnotetext{
${ }^{2}$ Detection rates were lowest in the conjunction condition (see Figure 9C).
} 

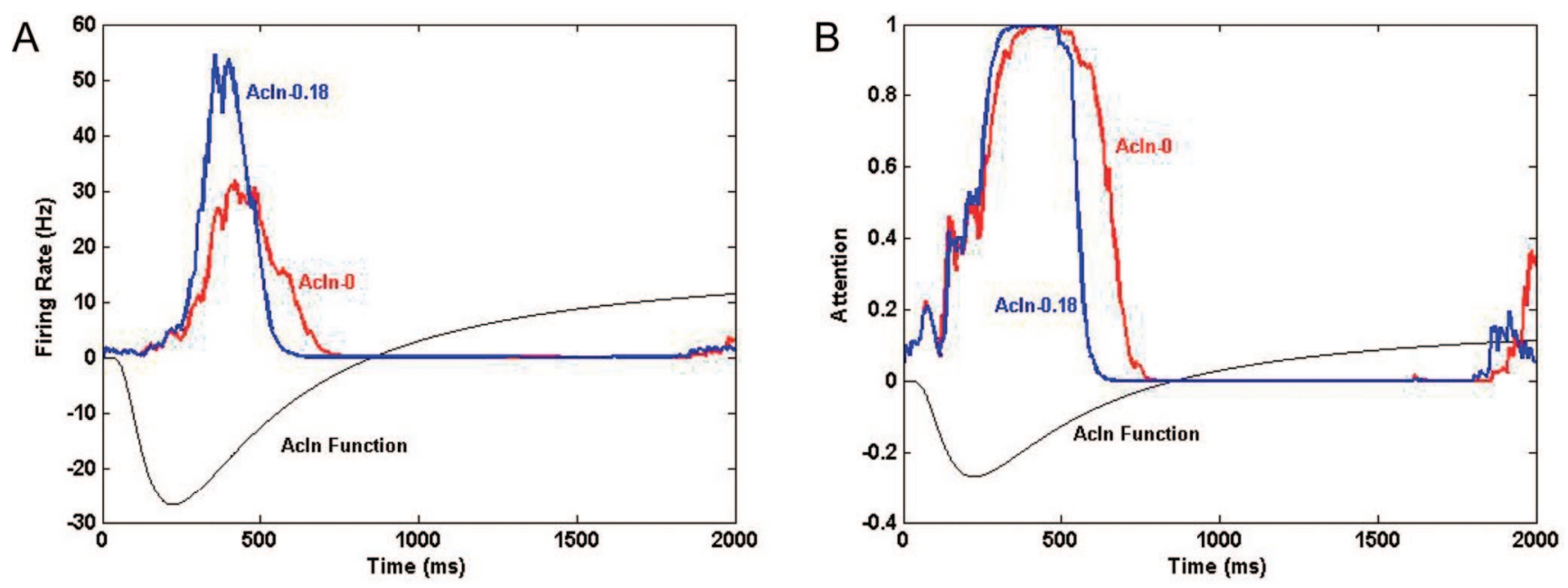

Figure 10. A: The effect of top-down inhibition on firing rate activity in the location map. B: The profile of "attention" to an initial distractor (reflecting the contrast between activity at the critical position and other positions in the location map). The bottom (black) line indicates the inhibition function over time. The blue line indicates the firing rate of neurons when there is active inhibition present. The red line indicates activity when there is no active inhibition present. There is a delay in frequency adaptation when inhibition decreases.

distractors relative to the new items presented in the search displays, making search as efficient as the single-feature baseline (when only new search items are presented). Under the parameters set for an interval of 1,000 ms, this suppression effect extends out to intervals of around $2 \mathrm{~s}$ (see Figure 10) and could be extended for longer if inhibition is reapplied. The sSoTS model captures the time course of preview search based on the assumed, biologically plausible temporal parameters of neural adaptation. The sSoTS model provides a qualitative fit to the data on human search. Note

Table 1

Clinical Details of the Patients Tested in Study 5A

\begin{tabular}{|c|c|c|c|}
\hline Patient & Sex & Age & Clinical deficit \\
\hline \multicolumn{4}{|r|}{ Right PPC patients } \\
\hline JB & $\mathrm{F}$ & 74 & $\begin{array}{l}\text { Left hemiplegia, some neglect symptoms in } \\
\text { reading; left extinction }\end{array}$ \\
\hline MC & M & 62 & Left hemiplegia, left neglect and extinction \\
\hline $\mathrm{PF}$ & $\mathrm{F}$ & 59 & $\begin{array}{l}\text { Mild aspects of Balint's syndrome, left } \\
\text { extinction }\end{array}$ \\
\hline GK & M & 64 & $\begin{array}{l}\text { Balint's syndrome, left neglect and } \\
\text { extinction }\end{array}$ \\
\hline TM & M & 73 & Left hemiplegia, left neglect and extinction \\
\hline MP & M & 64 & $\begin{array}{l}\text { Left upper hemiplegia, left neglect and } \\
\text { extinction, mathematics problems }\end{array}$ \\
\hline $\mathrm{RP}$ & M & 58 & Left neglect and extinction \\
\hline $\mathrm{BS}$ & M & 64 & Left hemiplegia, neglect, and extinction \\
\hline \multicolumn{4}{|c|}{ Left hemisphere/occipital patients } \\
\hline HJA & M & 82 & $\begin{array}{l}\text { Visual agnosia, upper visual field defect, } \\
\text { alexia }\end{array}$ \\
\hline SA & $\mathrm{F}$ & 61 & Visual agnosia \\
\hline $\mathrm{BG}$ & M & 66 & Right hemianopia \\
\hline MH1 & M & 56 & Optic ataxia, right extinction \\
\hline MH2 & M & 68 & Right extinction, alexia \\
\hline DT & M & 68 & Right extinction \\
\hline
\end{tabular}

Note. $\quad \mathrm{PPC}=$ posterior parietal cortex $; \mathrm{F}=$ female $; \mathrm{M}=$ male. that absolute RTs in the model are somewhat shorter than in human search, but this may reflect the operation of additional factors that add a constant increment to human performance, such as the need to generate a motor response.

Effects of secondary tasks. Watson and Humphreys (1997) provided evidence that top-down processes in preview search are actively employed in order to optimize visual search over time, because the preview benefit was reduced under conditions in which a secondary task was imposed during the preview period (participants had to monitor for a central target letter whilst simultaneously ignoring the initial distractors). ${ }^{3}$ Watson and Humphreys proposed that the secondary task reduced top-down processes applied to the search task prior to the search display appearing. To assess this in sSoTS we have examined search when both the two top-down processes, top-down excitation (preactivating the features of expected targets) and top-down inhibition, were reduced to mimic the secondary task effect. The top-down parameters were both changed the same small amount (parameter reduction of 0.02), keeping all other parameters constant. Illustrative activation profiles in the location map of sSoTS are depicted in Figures $14 \mathrm{~A}-\mathrm{C}$ for changes in top-down bias, active inhibition, and a combination of both factors. Note that, in each case, there is increased competition for targets from activation in feature maps excited by distractors. The search results are presented in Figure 15. Search efficiency was reduced both when top-down excitation decreased (Figure 15A), when top-down inhibition decreased (Figure 15B), and when both contributions decreased (Figure 15C). The slope of preview search varied from $17.09 \mathrm{~ms} / \mathrm{item}$ (standard

\footnotetext{
${ }^{3}$ Humphreys et al. (2002) reported equivalent effects from an auditory monitoring task, demonstrating that preview search suffers under conditions in which other tasks compete for resources and not simply from factors such as masking when a visual secondary task is employed.
} 


\section{Preview Search}

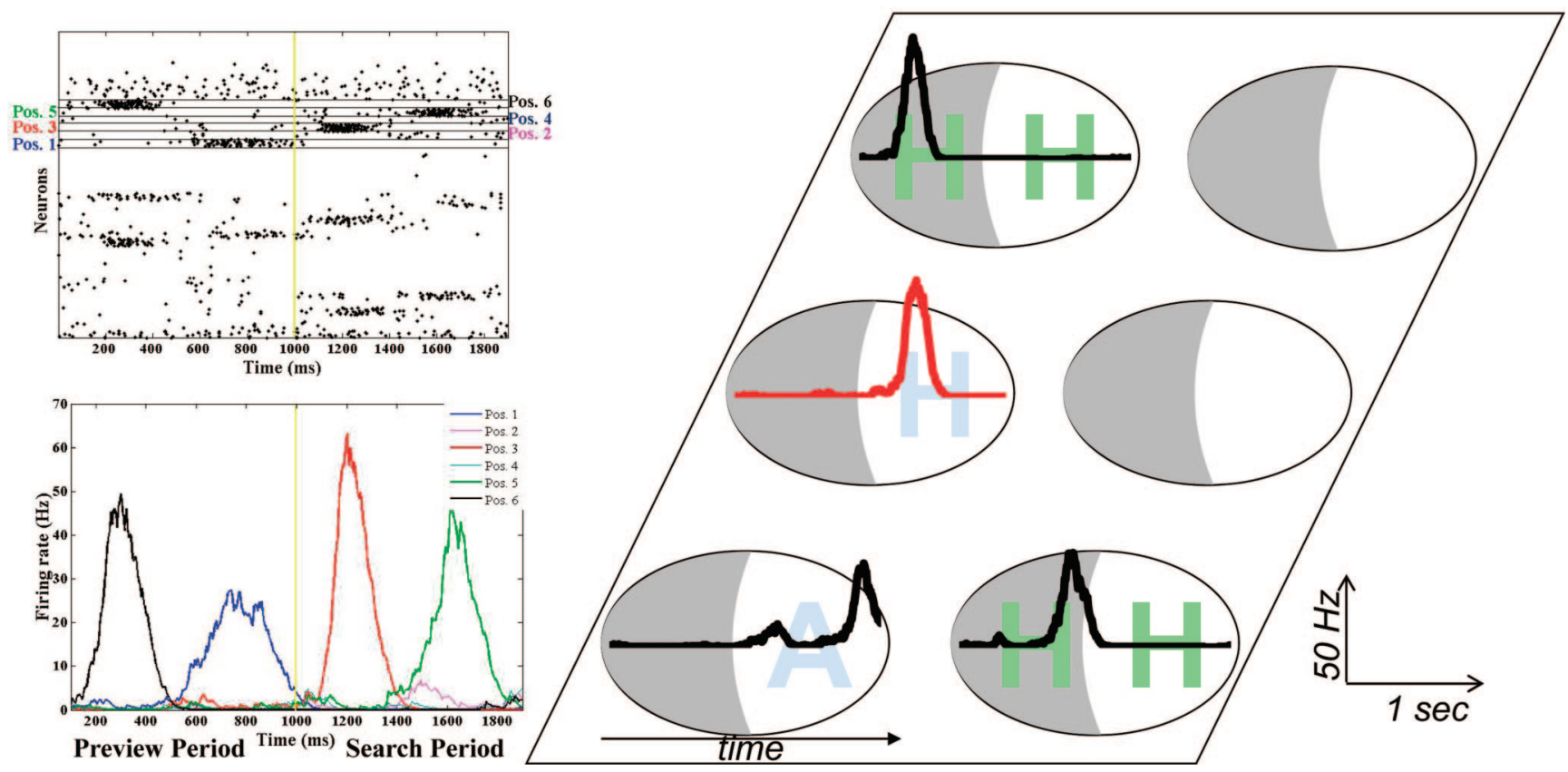

Figure 11. Activity within the clusters of units in the location map of the spiking selection over time and space (sSoTS) model in the preview condition. On the right, the gray area within each cluster indicates the time when the preview was present, and the white area represents the time when the search display was present. On the left, the activity profiles are shown in a combined graph for all positions (target in Position 3, red) in the form of spikes (top) and firing rate (bottom).

setting) to $30.8,29.73$, and $31.79 \mathrm{~ms} /$ item under conditions where there were decreases in top-down excitation, inhibition, and both factors. Changes isolated to the top-down parameters simulated the qualitative shift in search slopes under dual-task conditions.

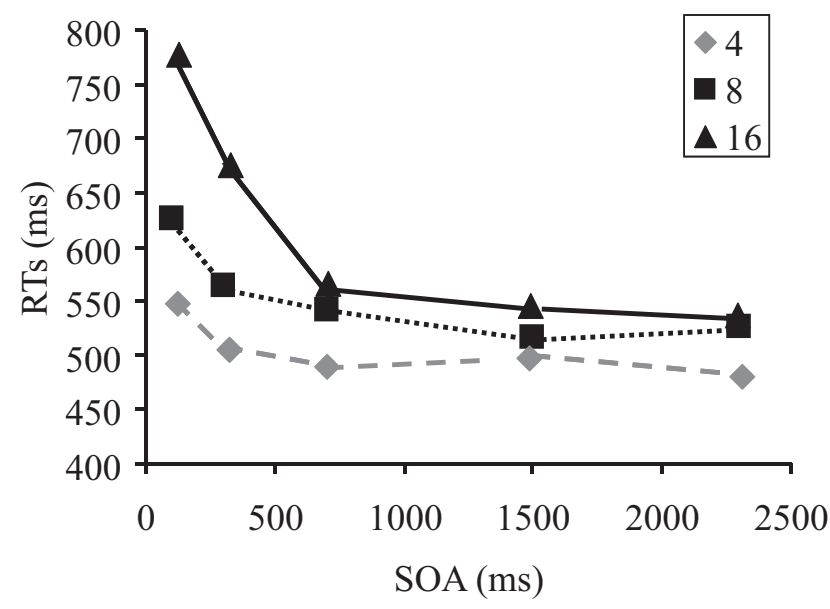

Figure 12. Data on human preview search (blue $H$ target vs. old green $H \mathrm{~s}$ and new blue $A$ distractors) as a function of the interval from the start of the preview until the start of the search display (the stimulus onset asynchrony $[\mathrm{SOA}])$. The zero condition here represents a conjunction search task, where all the items appear simultaneously. There were three display sizes with four, eight, or 16 items in the final display (adapted from Humphreys, Kyllingsbaek, et al., 2004). RT = reaction time.
These results show that sSoTS can simulate both the time course and the effects of secondary tasks on preview search. The full time course is captured by parameters set for a single preview duration, whereas secondary task effects are captured by variation in just the top-down parameters. Figure 16 presents predictive simulations

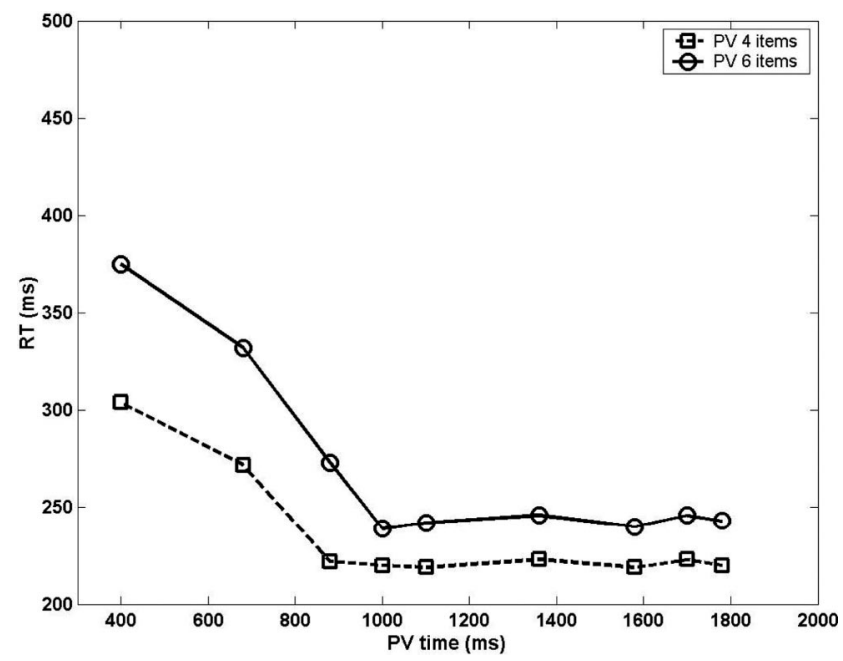

Figure 13. Simulations from the spiking selection over time and space (sSoTS) model of preview (PV) search as a function of the duration of the preview prior to the search display, for displays with a final total of four or six items. $\mathrm{RT}=$ reaction time. 

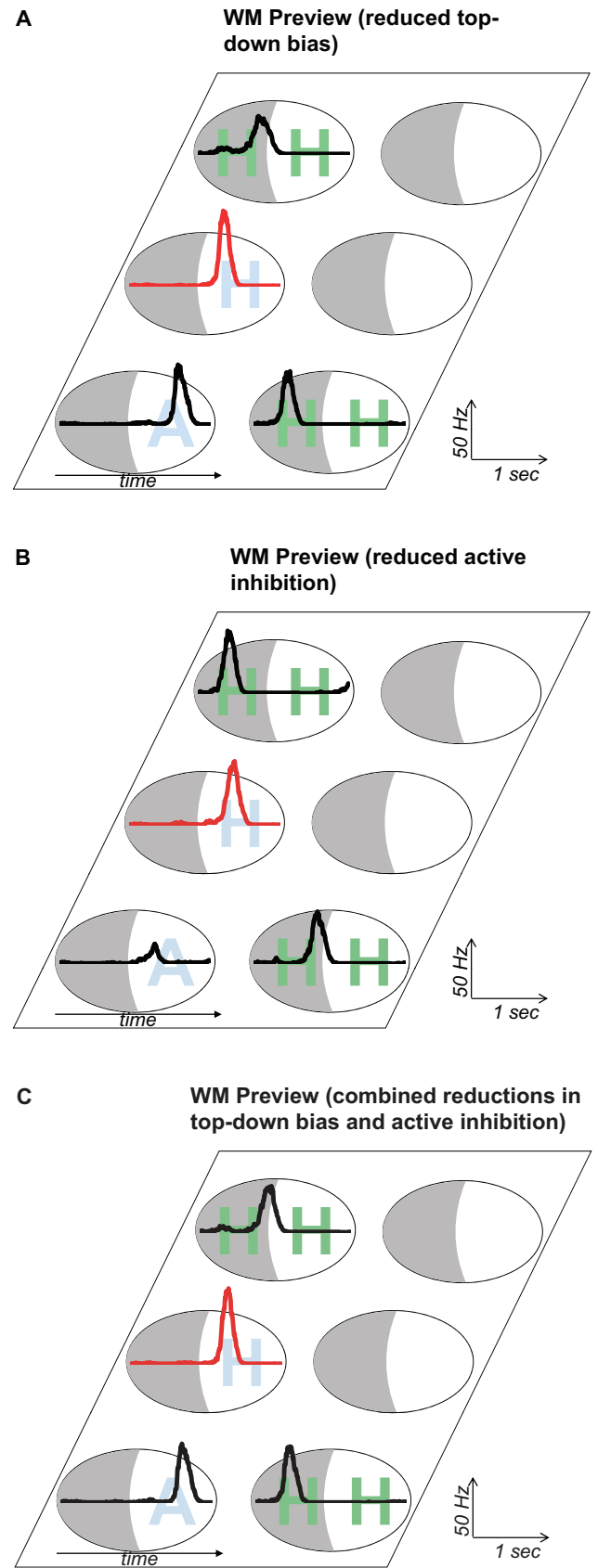

Figure 14. A: Activation in the clusters of units at each position of the location map of the spiking selection over time and space (sSoTS) model, for the preview condition with reduced top-down (excitatory) guidance to the target. B: Activation in the location map for preview search with reduced active inhibition. C: Activation in the location map for preview search when there is a combination of reduced excitatory guidance and reduced active inhibition. $\mathrm{WM}=$ working memory.

from the model when these factors are combined but the parameters are otherwise kept constant-when different durations of preview are presented and a dual-task load is imposed throughout (with reduced top-down biases). These data show two results. First, as we have noted previously, reducing top-down biases (under dual-task conditions) generates a cost in terms of search efficiency. Second, the effects of weaker top-down biases are greatest under short preview presentation conditions, and the costs are largely eliminated if the preview presentation time is extended. This is interesting because we modeled performance with topdown biases reduced throughout the preview period. Active inhibition accounts of preview search have not proposed a role for the frequency adaptation process present in sSoTS (e.g., Watson \& Humphreys, 1997). These theories predict that preview search should continue to be disrupted when participants remain engaged on a secondary task during the preview period, if that secondary task continues to consume the attentional resources required to inhibit the old distractors. However, for sSoTS, preview search becomes efficient as the preview duration is extended due to the second factor, frequency adaptation. According to the model, frequency adaptation takes longer when top-down processes are reduced, because previewed items gain in strength more slowly when top-down biases decrease, but adaptation should still eventually take place. In Study 5 we test the predicted time course of human search under dual-task conditions. Note that, here, sSoTS's prediction of efficient search at the long preview durations reflects the biologically plausible frequency adaptation process incorporated into the model.

Dual-task time course. It is also possible to assess performance as a function of the interplay between phases of preview search in which secondary tasks either are or are not introduced. We tested sSoTS when a "top-up" preview was presented without a secondary task, followed by a period when there was a secondary task. Watson and Humphreys (1997) examined human performance in a similar situation, when participants were given a top-up preview without any memory load and then a secondary task (monitoring for a target at fixation) was imposed while the preview remained present. Subsequently the search display appeared and the secondary task stopped. Watson and Humphreys found that engaging participants on the secondary task after the initial standard preview was sufficient to disrupt search to a level equivalent to that when no preview was initially presented. They suggested that, as soon as resources were withdrawn from the preview, there was reduced inhibition of the old items. However, they did not examine performance across a range of preview/preview + dualtask intervals, and it is not clear that Watson and Humphreys's conclusion stands across other conditions. We tested this in predictive simulations of sSoTS by evaluating the model in two conditions, with alteration only of the parameter reflecting the degree of top-down bias: (a) with reduced top-down inhibition through the preview period (for various durations, see above), to simulate performance when a dual task was continued through the preview, and (b) when a top-up preview, presented under standard conditions (with the usual top-down biases applied for $500 \mathrm{~ms}$ ), occurred before the different lengths of preview exposed under dual-task conditions (when top-down biases were reduced).

The results are shown in Figure 17. According to these simulations, there is little benefit from the top-up (standard) preview when the duration of the preview + secondary task is relatively short (here, $500 \mathrm{~ms}$ ). However, a benefit from the initial top-up does emerge as the duration of the preview + secondary task increases (with durations of 900 and 1,000 ms for the preview + secondary task). This result occurred even though the initial preview top-up was not itself sufficient to generate a maximum 

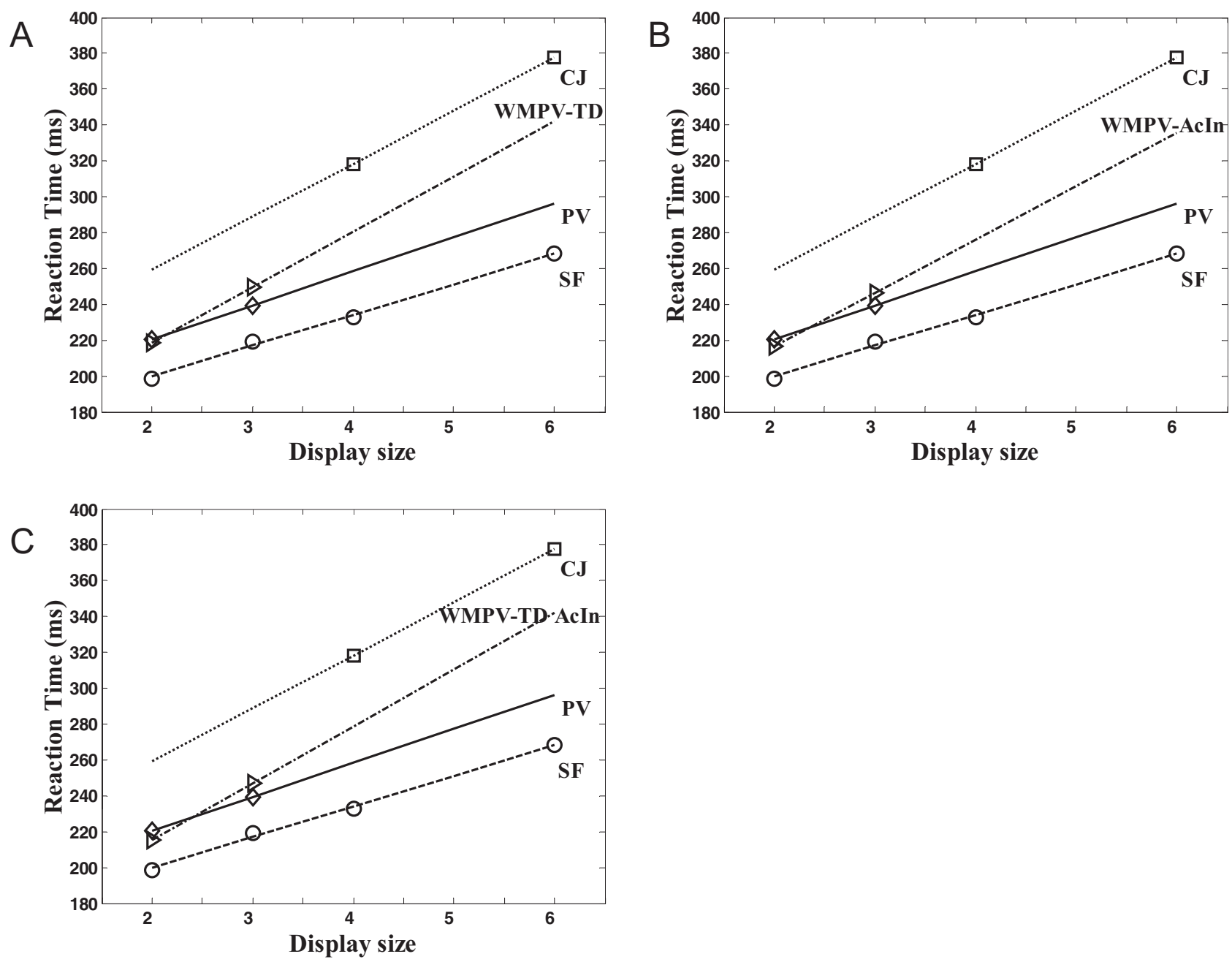

Figure 15. A: Simulation of search under conditions of reduced top-down excitatory guidance. B: Reduced active inhibition. C: A combination of both A and B.

preview benefit when presented alone (i.e., it was not sufficient to "push" previewed items into their refractory state; see Figure 13, where the initial top-up period is covered)—but the preview duration was clearly sufficient to move activation toward the refractory state, so that subsequent effects of reduced inhibition were less detrimental to performance. Unlike the high-level psychological account offered by Watson and Humphreys (1997), sSoTS holds that preview effects can emerge even when resources are withdrawn from the preview, but this depends on the initial "state" of participants and how far along the attentional system is toward adaptation taking place.

Predictions 2 and 3. These simulations with sSoTS lead to two novel predictions. One is that, even when participants engage in a secondary task during the preview period, a preview benefit should emerge if the preview is presented for long enough (see Figure 16). The second prediction is that, if a top-up of the preview is given before a secondary task is imposed, there should be a gain to performance-but this emerges only as the duration of the preview + secondary task increases (see Figure 17). In each case there is not a simple loss of search efficiency if resources are withdrawn during the preview period (contra to the claim of Watson \& Humphreys, 1997). These novel predictions, based on the biologically plausible temporal dynamics of the spiking-level model, were assessed in Studies 5B and 5C.

\section{Study 3: Simulating Effects of Brain Lesions}

Classically, disorders of visual attention are associated with clinical impairments such as unilateral neglect that are complex and multifaceted, with behavioral dissociations occurring across and sometimes even within patients (e.g., Humphreys \& Riddoch, 1994). Computational modeling offers the possibility of providing an overarching account of these disorders, with the behavioral deficits emerging after damage to different components of the modeled attentional system. There have now been several attempts to simulate visual neglect, typically using relatively high-level connectionist approaches (Heinke \& Humphreys, 2003; Mozer, Halligan, \& Marshall, 1997). These models have typically aimed at capturing the spatial biases that characterize the syndrome without exploring wider aspects of lesioning, where neglect may emerge in 
the context of other deficits. One interesting possibility offered by spiking-level models is that some of the nonspatial deficits associated with neglect may stem from basic biological constraints on information processing. These constraints can be captured by such models.

Within the framework of a spiking-level model, a neurological insult such as a stroke can be modeled very simply (and plausibly) by removing the number of neurons available within a given pool. This will have several consequences. One is that it will generally add to the level of noise operating within the system, because noise is a function of the number of neurons present within the network

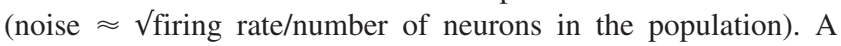
natural prediction, then, is that the performance of patients will be more variable than that of control participants, as neurons are removed from the model to create the lesioned state. Figure 18A presents data from patients with posterior parietal lesions on formcolor conjunction search and matched single-feature search tasks (for a form and a color-defined target), for targets in their contraand ipsilesional fields. ${ }^{4}$ It is evident that there is an increase in the variance in the contra- relative to the ipsilesional field, and this was greatest for the conjunction condition.

We have simulated these generic effects of lesioning in the sSoTS model. The model was lesioned by removing a percentage of the neurons in the pools representing the left side of the location network (Mavritsaki et al., 2009) but keeping the other parameters constant. The model was run with different random seeds for the Poisson noise in the firing rates, and with an average of $10 \%$ of the neurons on one side lesioned (how many units were lesioned was varied randomly). This lesioning level was selected by reducing the number of neurons until some change in the behavior was observed. Figure 18B illustrates that the lesioned model showed an increase in variability in the contra- relative to the ipsilesional

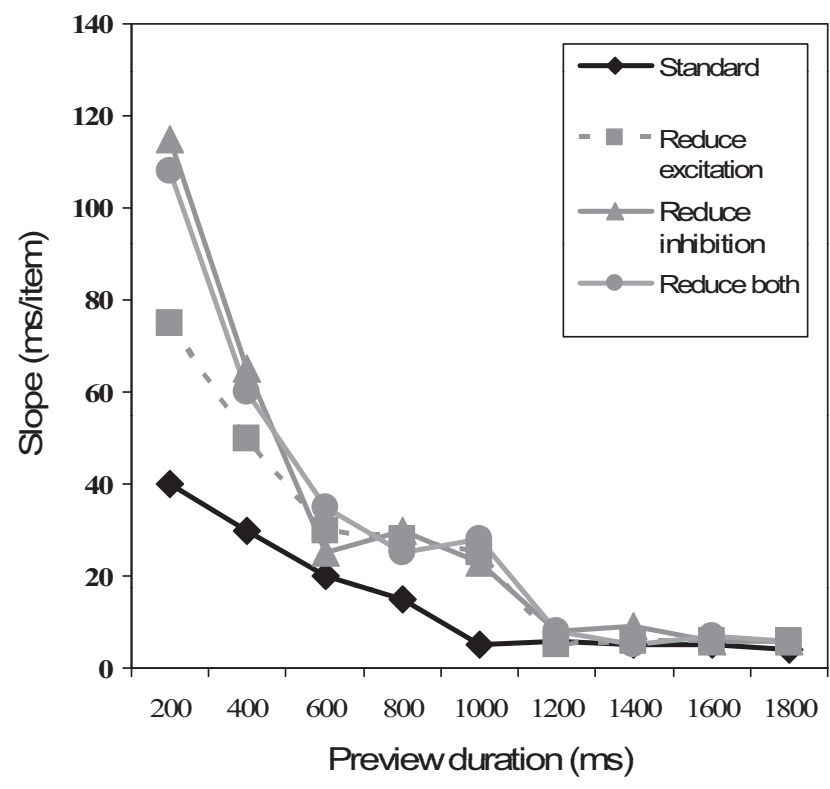

Figure 16. Predictions from the spiking selection over time and space (sSoTS) model concerning the slope of the search function (ms/item) as a function of the preview duration under conditions of a secondary task load (with reduced top-down guidance, active inhibition, or a combination).

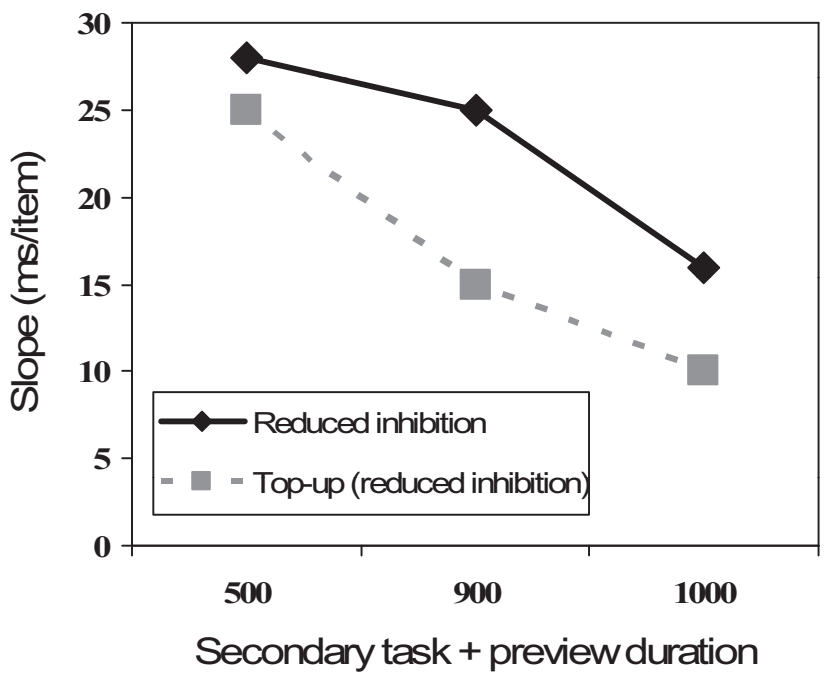

Figure 17. Predictions from the spiking selection over time and space (sSoTS) model for the slope of the search function (ms/item) as a function of the preview duration given under secondary task conditions (the reduced inhibition condition) and when the same conditions are preceded by a top-up preview $(500 \mathrm{~ms})$ prior to the secondary task period.

field, particularly in conjunction search. The effect emerges in the model because reducing the number of neurons present increases the level of noise present, and this leads to greater variance across trials. The effects of noise are particularly influential when there is greater competition for selection (for conjunction relative to single-feature search). This points to the importance of looking at the variance as well as the mean levels of performance in neuropsychological patients - an issue that emerges naturally when lesioning at the spiking neuron level but that is not suggested directly by higher level simulations.

As noted in Study 1, brain lesions can have specific effects at a microgenetic level; Posner and Petersen (1990) argued that there is localized control of arousal systems within the right hemisphere of the brain through lateralization of the norepinephrine regulation system. This can decrease neurotransmitter function generally, so that there is a performance detriment on both sides of space. They suggested that this was a contributory factor to neglect being more prevalent after right than left hemisphere damage (cf. Heilman \& Valenstein, 1979). Consistent with this, Malhotra et al. (2005) found that the deficits in spatial selection associated with neglect decreased when patients were administered a drug that increased excitatory neuron-transmitter function. To capture such effects, Mavritsaki et al. (2009) contrasted effects of (solely) removing neurons from pools on one side of the location map in SSoTS, with the effects of doing this plus varying the level of NMDA neurotransmitter within the model (mimicking changes associated with

\footnotetext{
${ }^{4}$ Data were collected over 12 patients with posterior parietal lesions, mean age 63 years. The display conditions matched those reported in Study 5. The single-feature task involved search for a blue $H$ target amongst blue $A$ distractors (form feature) or search for the same stimulus amongst green $H$ distractors. The same target was used in the conjunction task, but the distractors were half blue $A$ distractors and half green $H$ distractors. The single-feature data are averaged across the two feature targets.
} 


\section{Contralesional}

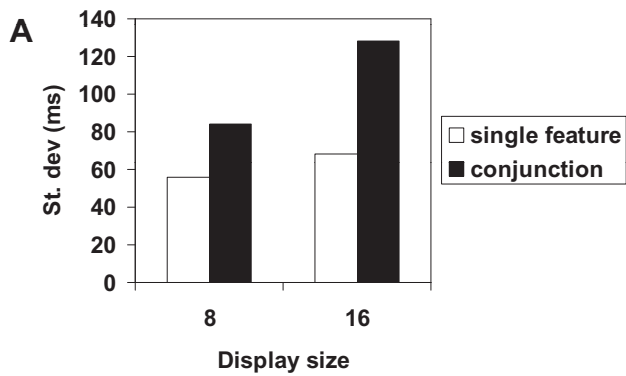

B

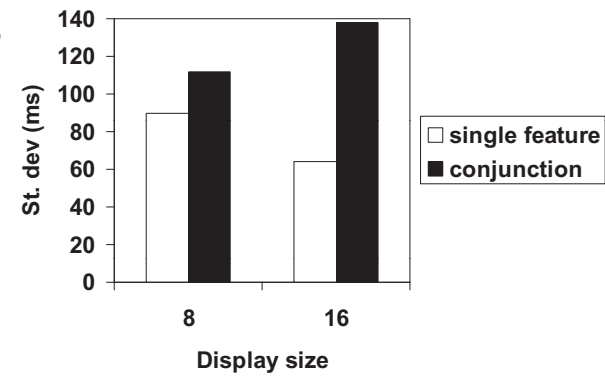

Ipsilesional
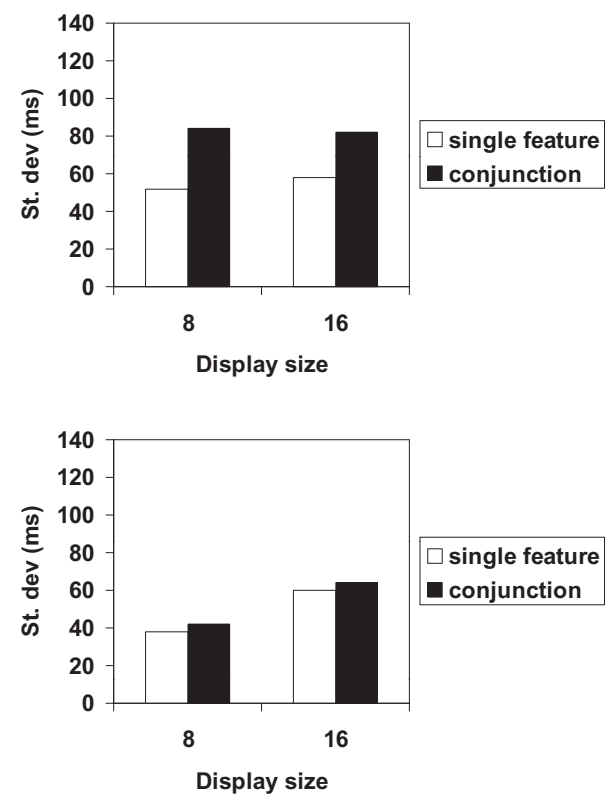

Figure 18. A: Data from patients with posterior parietal cortex (PPC) lesions showing the variability (st. dev) of reaction time in single-feature and conjunction search conditions, as a function of whether targets are presented in the contra- or ipsilesional field. B: Comparable data from the spiking selection over time and space (sSoTS) model when units on one side of the location map are lesioned.

damage to the norepinephrine system; Posner \& Petersen, 1990). Damage to the location map alone strongly affected the detection of targets on the impaired side of space. This deficit was more serious on conjunction search than on feature search, reflecting the greater competition for selection that characterizes conjunction search. This matches human neuropsychological data (Eglin, Robertson, \& Knight, 1990; Riddoch \& Humphreys, 1987). The same lesion, when combined with reduced neuromodulation of synaptic transmission, led to more marked impairments for a given degree of lesioning. The effect was to exaggerate the deficit with conjunction targets, especially when targets fell in the contralesional field. In addition to this, problems emerged in the detection of ipsias well as the contralesional targets (there was about a $27 \%$ decrease in performance for ipsilesional conjunction targets, with standard vs. reduced NMDA). These results are presented in Figure 19..$^{5}$ Thus, over and above the effects of increased noise, there may be selective effects on neuromodulation from altering the central regulation of neurotransmitter function. This factor emerges naturally from models that incorporate neurotransmitter functions while also predicting whole-system behavior (here, visual search). Changes in arousal have not been examined in higher level simulations of neglect.

\section{Time Course After Lesioning}

As well as simulating overall aspects of spatial search, as noise and neurotransmitter levels vary, sSoTS also simulates deficits in visual search over time. As we have noted, lesions of the PPC generate selective problems in preview search in humans, measured relative to a single-feature search baseline (Humphreys et al.,
2006; Olivers \& Humphreys, 2004). Lesioning the map of locations in sSoTS, even without additional changes in arousal, generates a deficit in preview search similar to that found in PPC patients, with search being particularly difficult for contralesional targets. Figure 20A presents the slopes of the search functions for the single-feature and preview conditions (efficiency/item) for a preview duration of 1,000 ms and an average $10 \%$ lesion on one side of the location map. There was a clear and selective loss of search efficiency for contralesional targets in the preview condition. In sSoTS this deficit in preview search arises because lesioning increased the time to accumulate information from the preview (especially on the lesioned side of the field), so that previews were not inhibited when the target appeared. In addition, there was a slowing down of target selection on the contralesional side. The net result was that there was increased competition for selection for contralesional targets. This increased competition is demonstrated in Figure 21 (note the activation for the pool of units corresponding to the old [blue $A$ ] distractors). Although competition increased across single-feature as well as conjunction and preview search, the effect of lesioning was greatest in the latter conditions due to the overlap of features across targets and distractors in conjunction and preview search.

The sSoTS results are consistent with lesions to the PPC leading to poor spatiotemporal segmentation of old previewed distractors from new search items. This spatiotemporal segmentation account contrasts

\footnotetext{
5 The data here are presented in terms of RT/proportion correct (Townsend \& Ashby, 1983), to provide a single processing efficiency measure where reaction times and errors are combined.
} 
Contralesional

A

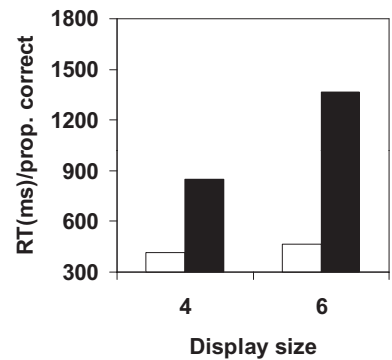

B

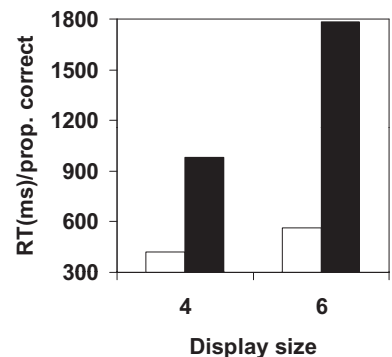

Ipsilesional
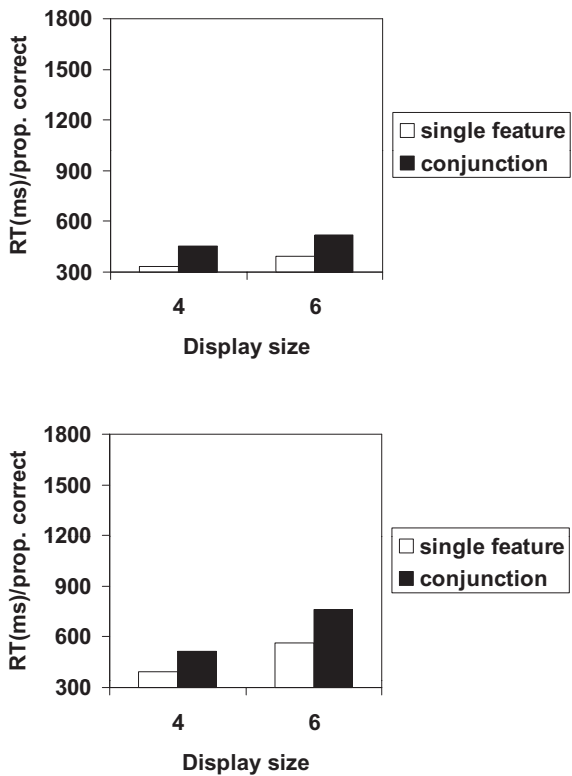

Figure 19. A: Data from patients with posterior parietal cortex (PPC) damage showing the efficiency of single-feature and conjunction search (RT/proportion correct) with contra- and ipsilesional targets. B: Simulations with the spiking selection over time and space (sSoTS) model after unilateral lesioning of the location map for single-feature and conjunction search with contra- and ipsilesional targets. RT = reaction time.

with other accounts of the effects of posterior parietal lesioning. For example, one influential account of PPC lesions is that they lead to impaired spatial disengagement of attention from the ipsilesional to the contralesional field (Posner, Walker, Friedrich, \& Rafal, 1984). According to this account, a deficit for contralesional targets in preview search may arise because patients have difficulty disengaging attention from ipsilesional distractors in the preview. Olivers and Humphreys (2004) discarded a spatial disengagement account, how- ever. This account predicts that performance should be most disrupted when the preview items fall in the ipsilesional field and the new search items in the contralesional field. In contrast to this, Olivers and Humphreys found that patients performed relatively well in that condition and suffered most when the old and new stimuli were spatially overlapping (especially when both the old stimuli and the target were presented on the contralesional side). Rather than fitting a disengagement account, these data are consistent with patients having

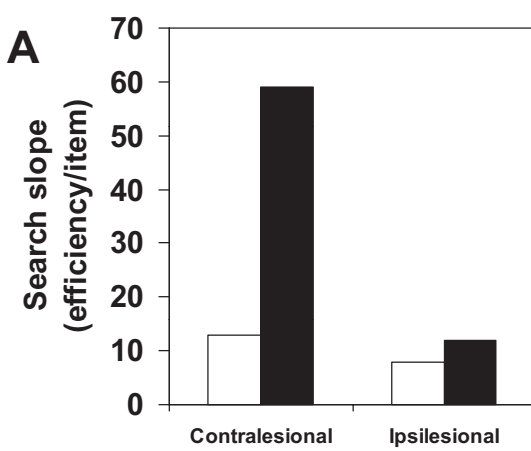

Field

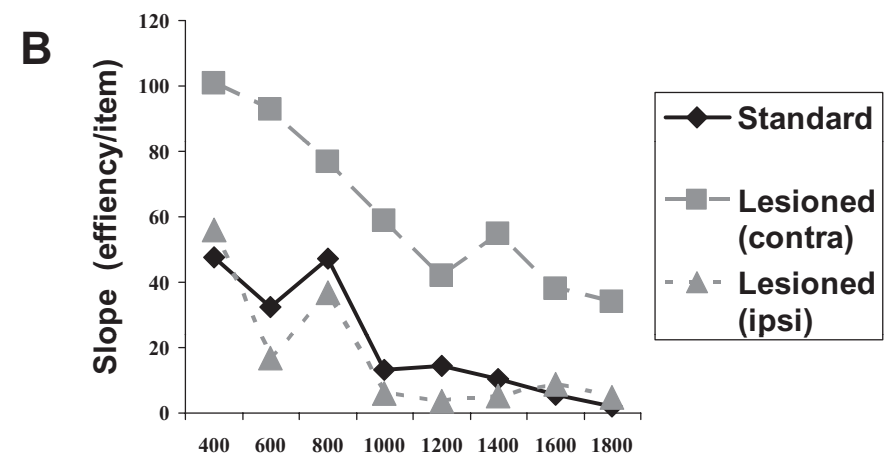

Preview duration

Figure 20. A: The slope of the search function (based on a measure of processing efficiency, RT/proportion correct) for the spiking selection over time and space (sSoTS) model after lesioning of the location map, for single-feature search (blue $H$ vs. blue $A$ s) and preview search (blue $H$ vs. green $H$ s and blue $A$ s). B: The slope of the search function (using an efficiency measure) for the standard and lesioned versions of sSoTS as a function of the duration of the preview. The slope for targets in the lesioned field reduces over time, but, across the time periods sampled, it does not reach the level found in the standard (nonlesioned) condition. 

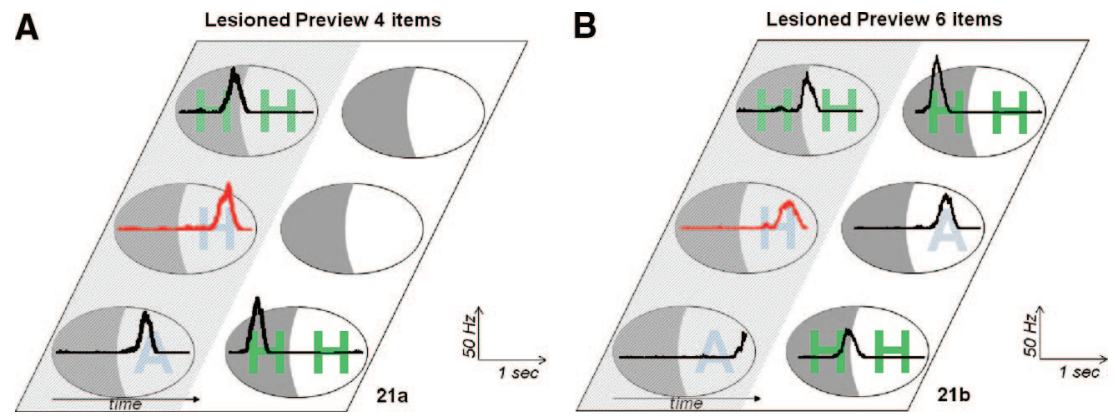

Figure 21. Activation in the location map in the lesioned version of the spiking selection over time and space (sSoTS) model in preview search (1,000-ms preview) for (A) four and (B) six items. The shading across the left side of the map indicates the lesioned area.

generally poor spatiotemporal segmentation, with the problems emerging most strongly when the time course of processing of targets and old distractors are both delayed (preview and target in the contralesional field). Mavritsaki et al. (2009) reported a simulation of this result.

To date, empirical studies of the effects of PPC lesions on preview search have examined performance only under preview durations that, in normal participants, produce an asymptotic level of performance. An examination of the time course of processing in the lesioned version of sSoTS is presented in Figure 20B. These simulations indicate that the deficit for contralesional targets can extend across a wide range of preview durations, including those at least double the durations required for normal participants to reach asymptotic search performance (e.g., previews of 1,800 ms, in Figure 20B). In addition, the model predicts that, for patients, the search slope for contralesional targets under preview conditions remains higher than that for ipsilesional targets, even when the slopes reach their asymptote. This contrasts with the results from our simulations of dual-task performance, where search does eventually reach the same asymptote as the standard (single-task) preview condition. This contrast between dualtask effects and lesioning arises because the basic activation within the location map of the model is affected by removing neurons but not by altering top-down activity prior to the preview. Due to the generally reduced activation, contralesional new items do not benefit so much from top-down activity (excitation and inhibition), which normally contributes to the competitive advantage for the target over distractors. The net result is that there remains a residual cost to contralesional targets in preview search, because an abnormal level of competition remains across even long time courses. In addition, there is heightened competition for selection from new items appearing in unlesioned areas of field. In contrast, under dual-task conditions the new items eventually gain a full advantage from any top-down bias, and there is not unbalanced competition across the visual field. As a consequence, performance reaches the standard asymptote once (delayed) adaptation takes place.

Prediction 4. The examination of lesioned performance across the time course gives rise to a fourth prediction, which is that PPC patients should show a residual deficit in preview search even if the preview is presented for a prolonged duration. Without running the detailed neural-level simulations, it is not clear that a difference between the effects of brain lesion and of dual-task load would be evident. We tested the new prediction from sSoTS for a different profile of lesioning effects over different preview time courses in Study 5D.

\section{Study 4: Simulating fMRI Data}

Cognitive analyses of the functional components making up a given processing system have, over the past 15 years, been complemented by imaging studies that generate neural-level data during task performance (e.g., using positron emission topography [PET] and fMRI). These studies essentially correlate neural activity (via the BOLD signal in fMRI) with effects of manipulated stimulus and task variables in order to identify which brain regions support which component processes. However, it is one thing to link activity in cortical regions to variables in tasks and another to identify the functional role of those regions in the task. Most commonly, investigators adopt an empirical approach to identifying the functional role of brain regions identified through imaging - for example, by manipulating an appropriate variable and measuring its joint behavioral and neural effects. Problems can arise though when a given variable affects more than one process - an example is preview search, because there is evidence for both inhibitory and excitatory components (suppressing distractors and biasing search to new targets) contributing to performance (e.g., Braithwaite et al., 2005). How can we distinguish areas linked to the inhibitory and the excitatory components of search?

Spiking-level models can simulate imaging (e.g., fMRI) as well as behavioral data, because synaptic outputs in different regions of the model can be combined with an assumed hemodynamical response (HDR) function (Glover, 1999) to predict the BOLD function measured in fMRI studies (see Corchs \& Deco, 2004; Deco, Rolls, \& Horwitz, 2004; Humphreys, Mavritsaki, Allen, Heinke, \& Deco, 2009; Stemme, Deco, Busch, \& Schneider, 2005; see Logothetis, 2008; Logothetis et al., 2001, for physiological support for this approach). Note that these simulations depend on having synaptic activity directly available to feed into the HDR function.

There have now been four studies of human visual search over time using the preview paradigm (Allen, Humphreys, \& Matthews, 2008; Humphreys, Kyllingsbaek, et al., 2004; Olivers, Smith, Matthews, \& Humphreys, 2005; Pollmann et al., 2003). In each case, preview search has been associated with increased activation of the PPC, including the superior parietal lobe and the precuneus. This activation of the PPC could reflect either inhibition of the 
preview (e.g., due to the activation of inhibitory interneurons), top-down excitation of target features, or both. These different possibilities can be prized apart by combining fMRI studies with computational modeling. For example, the sSoTS model uses both active inhibition (applied to the features of old distractors) and active excitation (applied to the features of the expected target) in order to optimize visual search over time. Now, let us suppose that the task is to search for a new blue vertical target that appears amongst new blue $A$ horizontal distractors and that follows a set of old green vertical distractors. In such a search task, excitation would be given to the blue and vertical feature maps (corresponding to the target's features), while active inhibition would be given to the green and vertical maps (corresponding to the features of the distractors in the preview). We can then extract activation in the model reflecting the positive and inhibitory biases:

(a) Active excitation $=$ (activation for target form - activation for distractor forms, summed across their respective feature maps $)+$ (activation for target color - activation for distractor color, summed across their respective maps)

$$
\text { (Vertical - Horizontal }+ \text { Blue }- \text { Green }) .
$$

(b) Active inhibition $=$ (activation for distractor form activation for target form, summed across their respective feature maps) + (activation for distractor color - activation for target color, summed across the respective feature maps)

$$
(\text { Horizontal }- \text { Vertical }+ \text { Green }- \text { Blue }) .
$$

The resulting activation, pooled across the maps, can then be convolved with the assumed HDR to generate the HDR reflecting active excitatory and inhibitory biases in the model, and the HDRs predicted by the model can be used as regressors in the analysis of the fMRI data. This does more than simulate an HDR by a specified model; it uses the activity in the model associated with a particular (excitatory or inhibitory) function to identify the role of the activation patterns in different brain regions. This was done by Mavritsaki, Allen, and Humphreys (2008, 2010), with the parameters of sSoTS set to match human behavioral data on singlefeature, conjunction, and preview search. Mavritsaki et al. (2008, 2010) found that distinct regions of the PPC and extrastriate visual cortex were independently correlated with the activity profile over time, for the two types of bias. The active inhibitory bias significantly predicted the HDR function in the precuneus, while the active excitatory bias significantly predicted the HDR function in more lateral parietal cortex (see Figure 22). Thus, activation patterns associated with particular functions in the model were able to separate distinct patterns of activation found in the fMRI data. These results suggest that the cluster of regions activation in the PPC under conditions of preview search may be involved in functionally distinct computations in human visual selection.

One noteworthy aspect of these simulations of fMRI data is that the activation in the model is not bound to one anatomical region within the model, as activity was pooled across the feature maps. Here the analogy from the model is not at an anatomical but at a functional level-functional activity representing the changing activity profile across feature maps linked to either top-down excitation or inhibition correlated with activity in particular brain

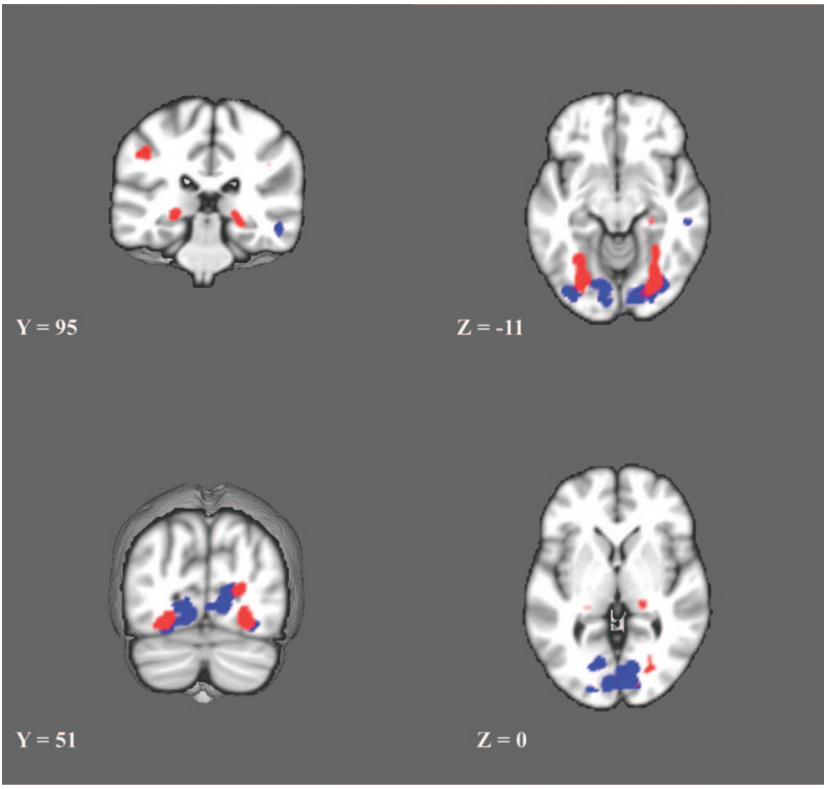

Figure 22. Brain areas where the blood-oxygen level-dependent (BOLD) signal in preview search showed significant correlation with either topdown excitation (red) or active inhibition (blue) in the spiking selection over time and space (sSoTS) model, with the excitation and inhibition values pooled across specific maps. From "Decomposing the Neural Mechanisms of Visual Search Through Model-Based Analysis of fMRI: TopDown Excitation, Active Ignoring and the Use of Saliency by the Right TPJ," by E. Mavritsaki, H. Allen, and G. W. Humphreys, 2010, NeuroImage, 52, p. 940 . Copyright 2010 by Elsevier.

regions. It follows that these brain regions may provide a source of the activation profiles evident in the feature maps in the model.

Mavritsaki et al. (2010) also carried out a more direct analysis of topological parallels between sSoTS and brain activity. To do this, activity within the location map was summed and convolved with the assumed HDR and then correlated with the brain activation recorded in an fMRI study of single-feature, conjunction, and preview search conducted by Allen et al. (2008). There was one reliable correlation, which was between the time course of neural activity summed across the location map and neural activity in search - with the correlation found in relation to BOLD activity in a region around the right temporoparietal junction (TPJ; see Figure 23). This result is of considerable interest, given that the modeldirected fMRI analysis was performed at a whole-brain level without any prior constraints. The right TPJ has been identified as playing a critical role in human visual selection, and lesions of this region are associated with clinical deficits in selection such as unilateral neglect. ${ }^{6}$ Corbetta and Shulman (2002) have argued that the right TPJ area serves as a "circuit breaker," signaling the presence of task-related stimuli in the environment that attention needs to be switched to. Shulman, Astafiev, McAvoy, Davossa, and Corbetta (2007) have further proposed that one role of the right TPJ is to filter out irrelevant distractors. The role of the location map in sSoTS bears some similarities to both ideas. In

\footnotetext{
${ }^{6}$ Note that Mavritsaki et al. (2009) simulated neglect by lesioning the location map in sSoTS.
} 
sSoTS, activation in the location map provides information about the likely presence of a target, which can then act as an input to other response-related systems that, for example, program an eye movement to that position. The pattern of activity in the location map also reflects the filtering-out of activity from distractorsthere is a sharper profile of activity in the location map when there is reduced competition for a target from the distractors (see Figure 6). It should be noted, though, that the activity used for these simulations was pooled across the locations within the map. Thus the observed correlation to activation in the human right TPJ does not necessarily indicate a topographic organization of the neurons in this region, but rather how target detection varies across time. It is also noteworthy that, in our analysis, activity correlated with the location map in the model was localized within the right hemisphere even though the search stimuli (including targets) were presented on both sides of space. This suggests that the location map may actually have bilateral receptive fields and be activated by stimuli on the right as well as the left side of space. Whatever the case, the simulations indicate that both functional (here, for excitatory and inhibitory processes in selection) and anatomical links (the TPJ and the location map) can be made on the basis of direct simulations of fMRI from neural-level models.

If the location map of sSoTS does mimic activation in the right TPJ, then we can derive neural-level predictions about how activation in this brain region may vary across different search conditions. For example, both experimental and modeling work has demonstrated that preview search is disrupted when participants

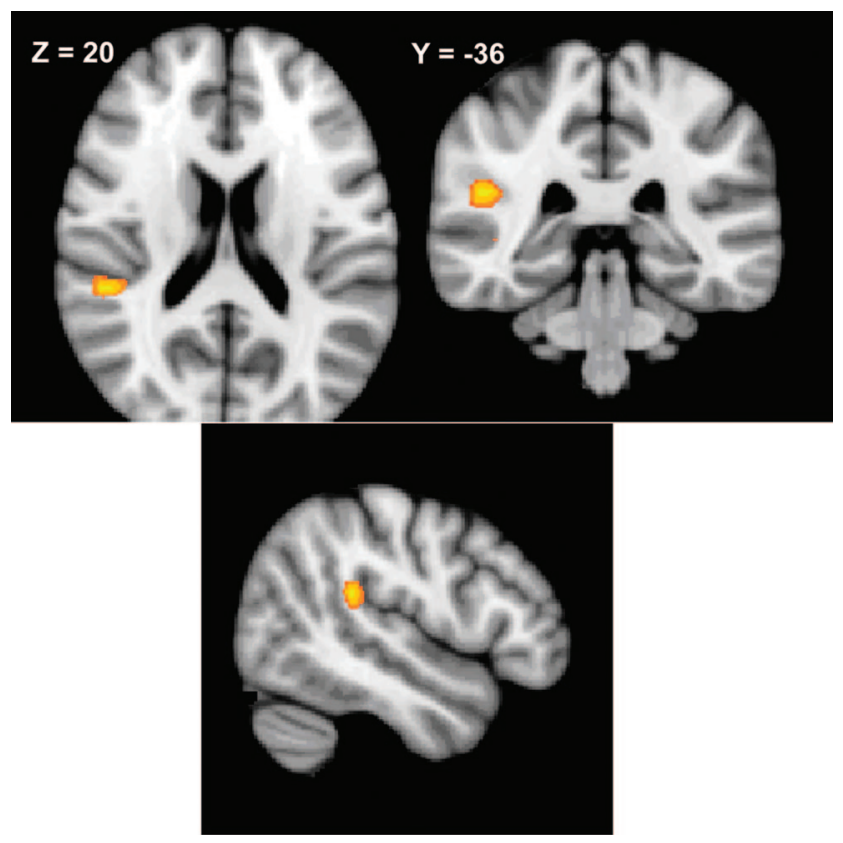

Figure 23. The brain region (right temporoparietal junction [TPJ]) where activity during search correlated with the hemodynamical response (HDR) derived from activity over time pooled across the location map of the spiking selection over time and space (sSoTS) model. From "Decomposing the Neural Mechanisms of Visual Search Through Model-Based Analysis of fMRI: Top-Down Excitation, Active Ignoring and the Use of Saliency by the Right TPJ," by E. Mavritsaki, H. Allen, and G. W. Humphreys, 2010, NeuroImage, 52, p. 940. Copyright 2010 by Elsevier. carry a working memory load during the preview (Humphreys, Jolicoeur, \& Watson, 2002; see Figure 15 for simulations here), which reflects increased competition for target selection from the old distractors when top-down influences on search are decreased. Under these conditions, the contrast between target and distractor activation is reduced (see Figure 24). If the right TPJ reflects this contrast, then activation in this region should decrease when search is carried out with a load through the preview period. Note that search itself becomes more difficult under these conditions, so a decrease in activation in the right TPJ would suggest that this region does not reflect search difficulty. Instead it would be consistent with the right TPJ reflecting target-distractor contrast (saliency).

Prediction 5. We predict that, relative to standard preview search, activation in the right TPJ should reduce when a load is introduced during the preview period. This was tested in Study 5E, where we reevaluated data from Allen et al. (2008), who used fMRI to study search in these two conditions.

\section{Study 5: Tests of the Predictions}

We have derived five novel predictions that emerge from the sSoTS model: (a) Impairments in selection can reflect general levels of arousal even when spatial biases are minimized in patients (Study 1); (b) there should be a prolonged time course of preview search under dual-task load conditions, but search can reach the same asymptote as under no load (Study 2); (c) there are benefits from giving a top-up preview prior to a preview being presented under dual-task conditions, and the benefits of this top-up should increase with increases in the exposure of the preview under dual-task conditions (Study 2); (d) in patients with lesions including the proposed location map, any deficit in detecting a contralesional target under preview conditions should decrease as the preview interval lengthens; however, search may never reach the same asymptote as that found in nonlesioned control participants; and (e) the BOLD signal in the right TPJ should be modulated when a dual-task load is introduced to preview search, but not in other search conditions. These predictions were evaluated in Study 5. These predictions emerge from the spiking-level simulations that attempt to incorporate biologically plausible parameters into the model.

\section{Study 5A: Selection and Deficits in Arousal/Sustained Attention}

In a first study we assessed selection in a case series of patients with posterior brain lesions and evaluated whether deficits in selection were related to changes in arousal/sustained attention. Posner and Petersen (1990) proposed that arousal/ sustained attention was modulated by a right hemisphere lateralized system and that activation of this arousal/sustained attention system was required in order to maintain attentional selectivity. Robertson and Manley (1999) extended this idea to propose that reduced arousal/sustained attention adds to the lateralized biases in selection after right hemisphere lesion, to generate symptoms of unilateral neglect-a disorder typically associated with right hemisphere lesions (Heilman \& Valenstein, 1979). The link between reduced arousal and poor selection was also apparent in Deco and Rolls's (2005a) simulations of single-cell recording data, where decreasing arousal (simu- 


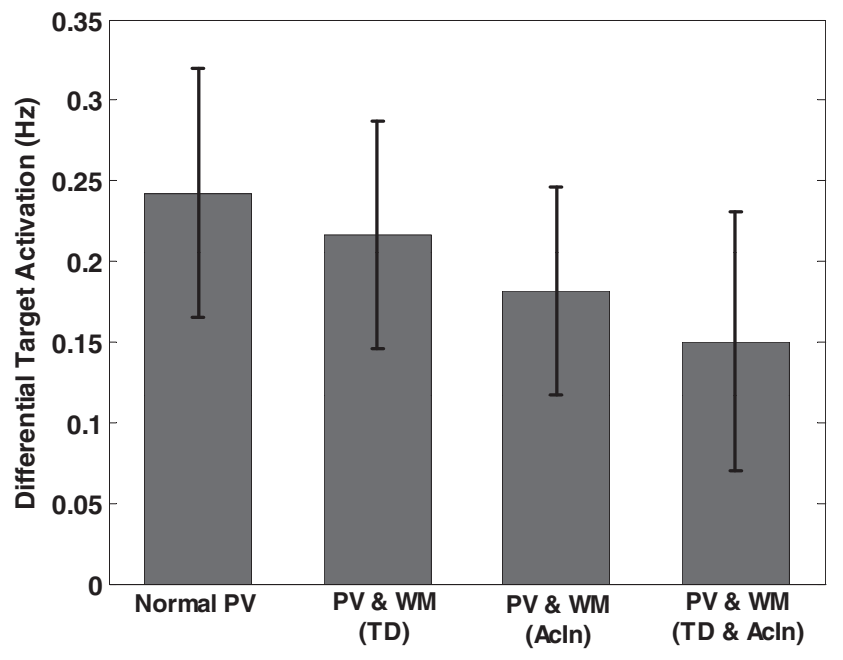

Figure 24. The differential rate of firing $(\mathrm{Hz})$ summed over units at the target location, compared with the rate over firing across distractor locations, within the location map of the spiking selection over time and space (sSoTS) model. Data are shown for the normal preview (PV; no load), when there is reduced top-down excitation (TD), reduced active inhibition (AcIn), and a combination of these factors (TD \& AcIn). WM = working memory. The error bars represent 1 standard error.

lated by reducing the effects of NMDA on the activation functions) disrupted the ability of the model to select a precued target. In Study 5A we tested whether poor selection in patients with posterior brain lesions was associated with poor arousal/ sustained attention. Selection was measured in terms of the contrast between reporting stimuli on trials with one and two items, and arousal was manipulated by providing an arousing but spatially noninformative tone prior to the occurrence of stimuli. To remove effects of lateral spatial biases on selection, we presented targets and distractors above and below fixation, and we tested whether patients with posterior right hemisphere lesions not only showed a selection impairment, but, specifically, whether their selection was modulated by changing arousal.

\section{Method}

Participants. There were 15 patients, all of whom had suffered strokes and all of whom had lesions involving posterior brain regions. Eight patients had damage that included the right PPC; seven had damage to either occipital regions or to left parietal cortex. Clinical details on the patients are presented in Table 1, and analyses of magnetic resonance imaging (MRI) scans are shown in Figure 25. In addition to the patients, a group of five age-matched control participants, without any history of brain lesion, was tested (age range $64-80$ years).

Apparatus and stimuli. A Pentium IV computer with an ATI RAGE PRO 128-MB graphics card controlled the stimulus displays and responses. The task was programmed and run on this computer using E-Prime (Schneider, Eschman, \& Zuccolotto, 2002a, 2002b). The stimuli were displayed on a Samsung SynchMaster 753 s color monitor. The monitor's resolution was $1024 \times 768$ pixels. The presentation of the stimuli was syn- chronized with the refresh rate of the monitor. Frame rate was fixed at $85 \mathrm{~Hz}$.

Design and procedure. The display was viewed from a distance of approximately $60 \mathrm{~cm}$. Each trial began with the presentation of a fixation cross for $500 \mathrm{~ms}$ that appeared alone (standard condition) or with a 200-ms tone at the onset of the cross (arousal condition). The 500-ms cue-target display interval was chosen here because prior work has indicated that this is an optimal time to change tonic arousal (Posner \& Boies, 1971). This was followed by a target display containing one or two letters, each $3^{\circ}$ of visual angle centered $3^{\circ}$ above or below fixation. The stimuli were the letters $A-D$, which occurred equally often on one- and two-letter trials and equally often above and below fixation. In both the standard and arousal conditions there were 48 one-item trials (24 above and 24 below) and 48 two-item trials. One- and two-item trials appeared at random, and the order of the standard and arousal conditions was randomized across participants. The task was to identify the letter. The stimuli were presented in white on a black background. The stimulus durations were $500 \mathrm{~ms}$ for all participants.

\section{Results}

The data for the patients are depicted in Figure 26. The controls scored at ceiling, with only 16 errors out of 480 on two-item trials (3.4\% error rate). Trials for the patients were scored as being correct or not and analyzed in a mixed-design analysis of variance (ANOVA) with the factors being arousal (standard vs. arousal condition), number of items (one vs. two), and patient group (right PPC patients vs. left hemisphere/occipital patients). There were reliable main effects of arousal, $F(1,13)=$ $40.4, p<.001, \eta_{p}^{2}=.757$, and number, $F(1,13)=110.29, p<$ $.001, \eta_{p}^{2}=.895$. There were significant interactions between arousal and patient, $F(1,13)=32.97, p<.001, \eta_{p}^{2}=.717$; arousal and number of items, $F(1,13)=17.39, p<.001, \eta_{p}^{2}=$ .572 ; and between arousal, number of items, and patient group, $F(1,13)=14.19, p<.01, \eta_{p}^{2}=.522$.

The three-way interaction was decomposed by analyzing the data for each patient group separately. The patients with damage to the right PPC showed reliable effects of arousal and number of items, $F(1,7)=164.61$ and 54.97, both $p \mathrm{~s}<.001, \eta_{p}^{2}=.959$ and .887 , respectively. The interaction was significant, $F(1,7)=$ $32.09, p<.001, \eta_{p}^{2}=.821$. There was no effect of the arousal condition on one-item trials $(t<1.0)$, but there was on two-item trials, $t(7)=-9.0, p<.001$.

The left hemisphere/occipital group showed only a significant effect of item number, $F(1,6)=61.74, p<.001, \eta_{p}^{2}=.911$, but neither the effect of arousal nor the Arousal $\times$ Number interaction were reliable, $F(1,6)=1.09$ and 2.55 , both $p$ s $>.05$.

We also compared the performance of the right PPC and the left hemisphere/occipital patients in each condition. Performance differed only in the standard condition on two-item trials, $t(13)=$ $-2.18, p<.05$.

\section{Discussion}

The patients performed worse than the controls, and all encountered some difficulty on identifying both letters on two- 


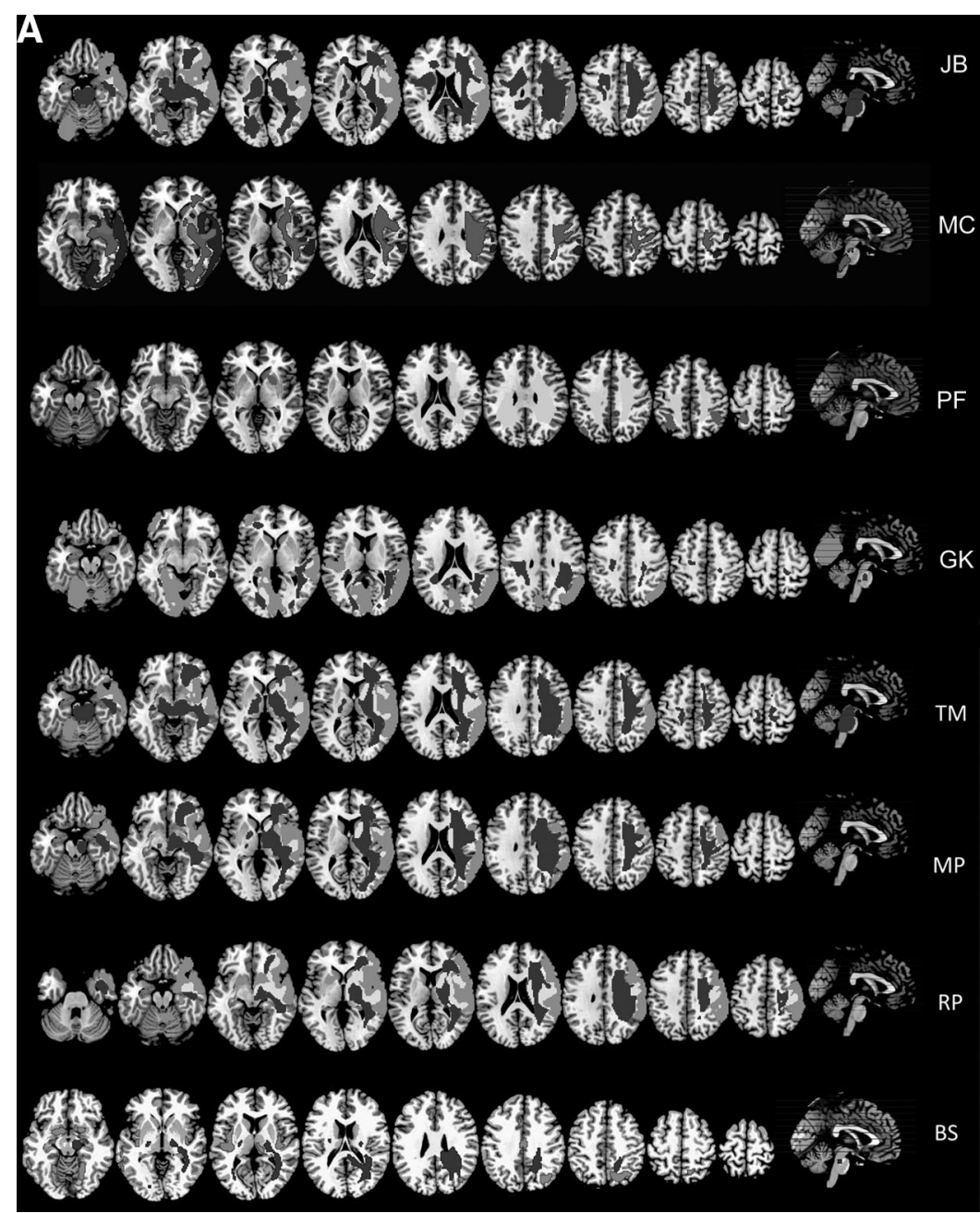

Figure 25. Lesion transcriptions for the patients tested in Study 5A. The figure depicts T1 structural scans (taken at 3T, with a 1-mm isotropic resolution). Colored-in light gray areas and colored-in dark areas reflect, respectively, changes in gray and white matter in the patients relative to scans from 201 control participants aged $40+$ years with no history of brain lesion. The changes were detected using voxel-based morphological analysis in SPM5 (http://www.fil.ion.ucl.ac.uk/spm/software/spm5/), and they are overlaid here on a standard multislice template in MRIcron. The images were first segmented into gray matter, white matter, and cerebrospinal fluid (CSF), and the resulting tissue class images were normalized without modulation (i.e., to compensate for the effect of spatial normalization). Images were smoothed with a Gaussian kernel of $2 \times 2 \times 2 \mathrm{~mm}$. The analyses are based on one-sample $t$ tests with three covariates: healthy gray/white matter versus patient gray/white matter, age, and gender. All areas are family-wise error corrected with $p=.05$ and an extent threshold specifying that only significant blobs containing $\geq 100$ voxels be included in the lesion. The panels represent patients with (A) posterior right hemisphere lesions or (B) posterior left hemisphere or occipital lesions.

item trials. The right PPC patients tended to perform better than the left hemisphere/occipital patients on one-item trials, whilst performing reliably worse in the standard condition with twoitem displays. This points to there being a deficit in selecting multiple items after right PPC damage. In addition to this, there was a selective effect of including an arousing auditory cue on the performance of the right hemisphere patients, who selectively improved report on two-item trials. There was no evidence for any consistent bias for reporting upper or lower field items, across the patients. These results are consistent with the selective deficit in the right PPC patients being linked to poor arousal, which is improved in the arousal condition. It is un- likely that this effect of the arousal cue was solely due to the relatively poor performance of the right PPC patients in the standard two-item condition. The four worst performing left hemisphere/occipital patients showed $0 \%$ improvement in the arousal condition, whereas four right PPC patients with a mean overall score matched to those of the worst left hemisphere/ occipital patients had a mean improvement of $15 \%$ on two-item trials in the arousal condition. The data point to differences in arousal rather than selection difficulty per se.

These results fit with Prediction 1 derived from the simulations of Deco and Rolls (2005a). There can be problems in selection linked to poor arousal that can be moderated by increasing arousal. 


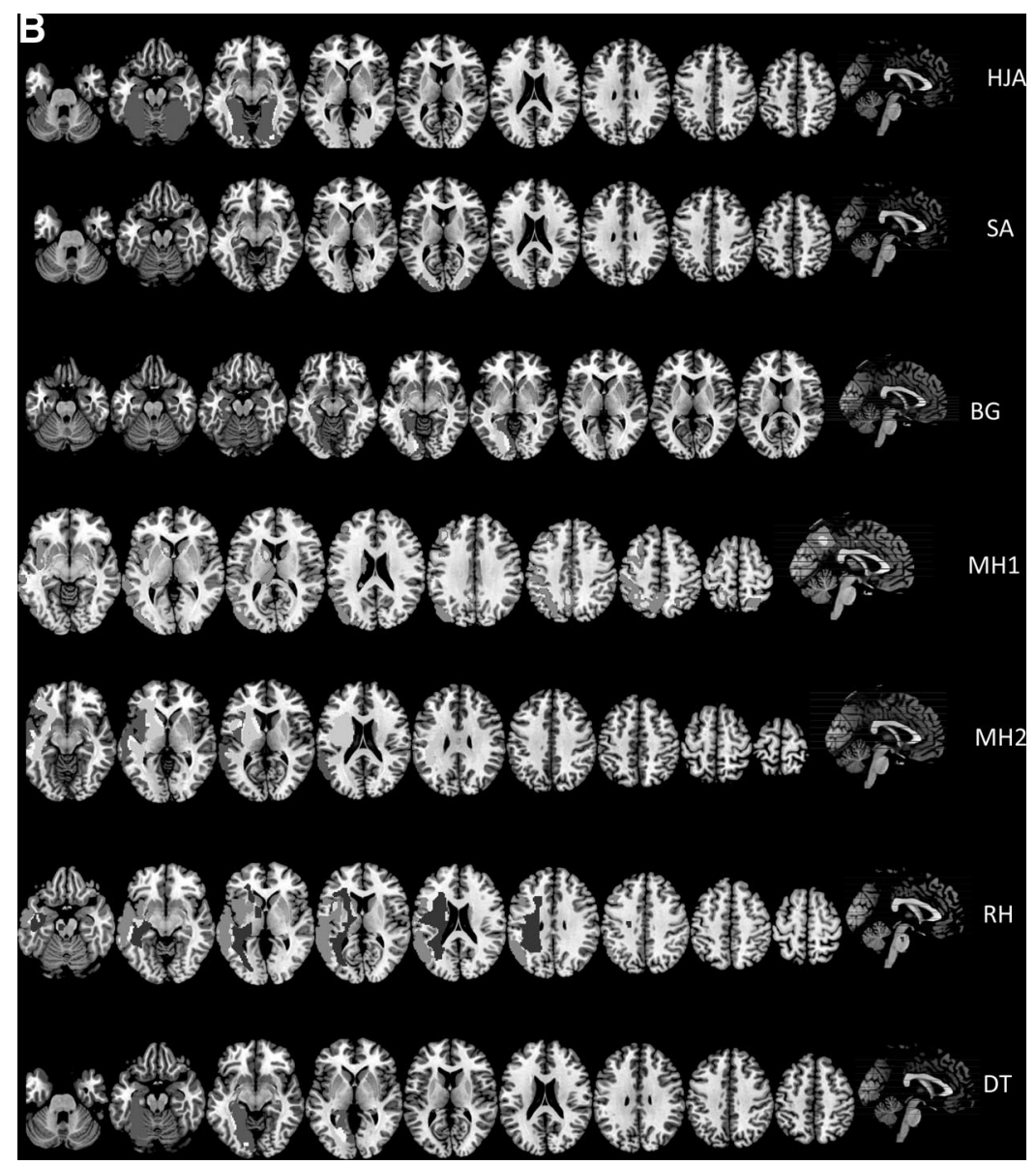

Figure 25. (continued)

The present data suggest that these deficits are encountered most notably after right PPC damage.

\section{Study 5B: The Time Course of Preview Search Under Load Conditions}

The sSoTS model also makes further predictions based on its time course of processing, especially under preview search conditions. One prediction is that, even though search efficiency decreases when a load is introduced through the preview, search should eventually reach the same asymptote as that found under conditions of no load. Prior behavioral studies of load effects have reported only decreased search efficiencies (e.g., Humphreys et al., 2002), but they may not have tracked a sufficiently long time period to enable the asymptote to be reached under load conditions. This was tested here by examining preview search under dual-task conditions across longer ranges of preview durations.

\section{Method}

Participants. There were eight participants aged between 20 and 25 years, four male and four female. All had either normal or corrected-to-normal vision.
Apparatus and stimuli. The displays were presented on a 15-in. monitor driven by a Pentium 200 PC with a VESA graphics card and running at a resolution of $800 \times 600$ pixels. The stimuli were generated by a purpose-written Turbo Pascal program (Version 7.0), which also recorded RTs and responses. Search displays were constructed by randomly plotting search stimuli within a virtual $8 \times 8$ grid, subtending approximately $8.3^{\circ} \times 8.3^{\circ}$ in visual angle (at approximately $75 \mathrm{~cm}$ ). The target was a blue $H$ and the distractors were blue $A$ s and green $H \mathrm{~s}$. The letters were rectangular and approximately $0.6^{\circ}$ high $\times 0.4^{\circ}$ wide. The green and blue colors (Commission Internationale d'Eclairage [CIE] values: 0.23, 0.38 for green; $0.26,0.32$ for blue) were selected as being very similar to those used originally by Watson and Humphreys (1997), set to equal luminance using a flicker fusion test on the experimenter (Ives, 1912).

Design and procedure. A trial began with a white fixation cross against a black background. In the single-feature and conjunction baseline conditions, this cross remained on the screen for 2,500 ms, which was the maximum length of the preview. On preview trials the cross appeared for $250 \mathrm{~ms}$ and was then replaced by a series of numbers that appeared at fixation consecutively for $250 \mathrm{~ms}$ each through the preview period. The numbers were selected at random with the exception that the number 1 could 


\section{Standard condition}

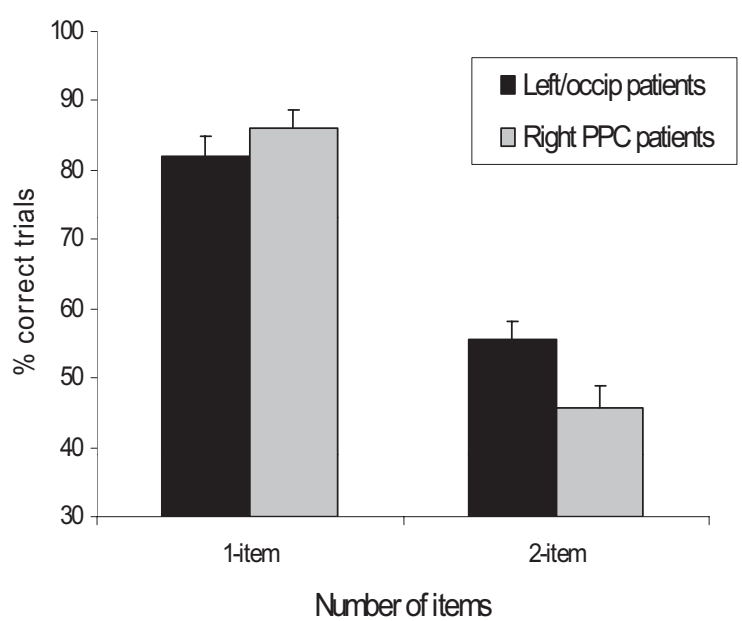

Arousal condition

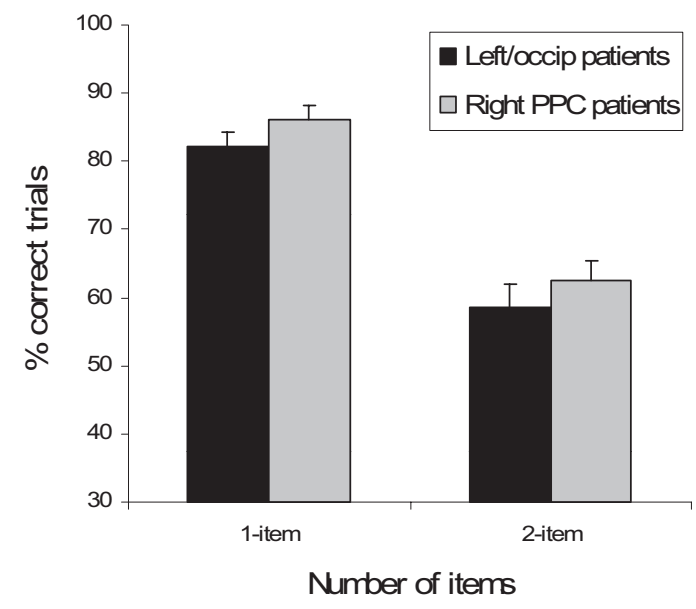

Figure 26. The percentage of correct reports on one- and two-item trials for right posterior parietal cortex (PPC) and left hemisphere/occipital patients in a standard selection condition (left) and in an arousal condition (right, with an auditory precue; Study 5A). The error bars represent $1 S E$.

appear only once on a trial. Participants were asked to try and detect whether a number 1 appeared. Prior studies have shown that this secondary task disrupts preview search (Watson \& Humphreys, 1997). There were four preview periods: 750, 1,000, 1,500, and 2,500 ms. When the preview is presented without an additional load task, search efficiency reaches an asymptote at the shortest interval used here (Humphreys, Kyllingsbaek, et al., 2004; see Figure 12 here). In addition to the letters at fixation, green $H$ stimuli were presented as a preview (either two, four, or eight items, randomly determined, on a trial). At the end of the fixation (single feature, conjunction) or load-task period (preview), the search displays were presented. In the conjunction condition the display contained four, eight, or 16 items. There was always a blue $H$ target present along with 2 green $H$ s and 1 blue $A$ (four-item display), 4 green $H$ s and 3 blue $A$ s (eight-item display), or 8 green $H$ s and 7 blue $A$ s (16-item display). In the single-feature condition, only the blue items were presented in the search displays (Display Sizes 2, 4, and 8). The green $H$ previews remained at the same locations, and search displays matching those in the single-feature baseline were presented. The task was to localize the blue $H$ target, which fell randomly either to the left or right of fixation. Responses were made by making a left or right mouse click, matching the left or right field for the target. In earlier studies of preview search, participants have made responses concerning either the location or a feature of the target that has always been present, and the pattern of data has been similar to that found on classic target present-absent tasks (e.g., Kunar, Humphreys, \& Smith, 2003; Olivers \& Humphreys, 2004). By presenting a target on every trial, we maximized the target-present data. Each of the six search conditions was blocked, and the order of the blocks was randomly assigned to the participants. There were 32 trials at each display size in each condition, leading to 576 trials per participant. Participants were instructed to remain fixated during the whole preview period.
Participants responded first to the location of the search target. In the preview conditions they were subsequently asked whether the target number 1 had appeared in the initial stream of numbers. Participants made a right mouse click if a number 1 had been present and a left click if it had been absent.

\section{Results}

The error rates were low and did not vary systematically across the conditions. The mean error rates are presented in Table 2 and were not analyzed further. The mean RTs are displayed in Figure 27. In Figure 27a the data for the preview and single-feature

Table 2

Percentage of Errors in Studies $5 B$ and $5 C$

\begin{tabular}{lrcc}
\hline & \multicolumn{3}{c}{ Display size } \\
\cline { 2 - 4 } \multicolumn{1}{c}{ Condition } & 2 & 4 & 8 \\
\hline & Study $5 \mathrm{~B}$ & & \\
Single feature & 1.6 & 1.2 & 2.0 \\
Conjunction & 0.8 & 3.5 & 2.0 \\
750-ms preview & 1.2 & 2.0 & 1.8 \\
1,000-ms preview & 2.7 & 3.5 & 3.5 \\
1,500-ms preview & 0.4 & 2.0 & 2.3 \\
2,500-ms preview & 0.8 & 0.8 & 4.7 \\
\hline & & & \\
Single feature & Study $5 \mathrm{C}$ & & \\
Conjunction & 3.1 & 2.7 & 3.1 \\
$350+750$-ms preview & 2.3 & 4.3 & 0.8 \\
$350+1,000-$-ms preview & 1.2 & 2.0 & 3.1 \\
$350+1,500$-ms preview & 0.8 & 3.1 & 3.1 \\
& 2.3 & 2.7 & 1.6 \\
\hline
\end{tabular}

Note. The data are shown as a function of the number of blue items present (i.e., half the items present in a conjunction display). 

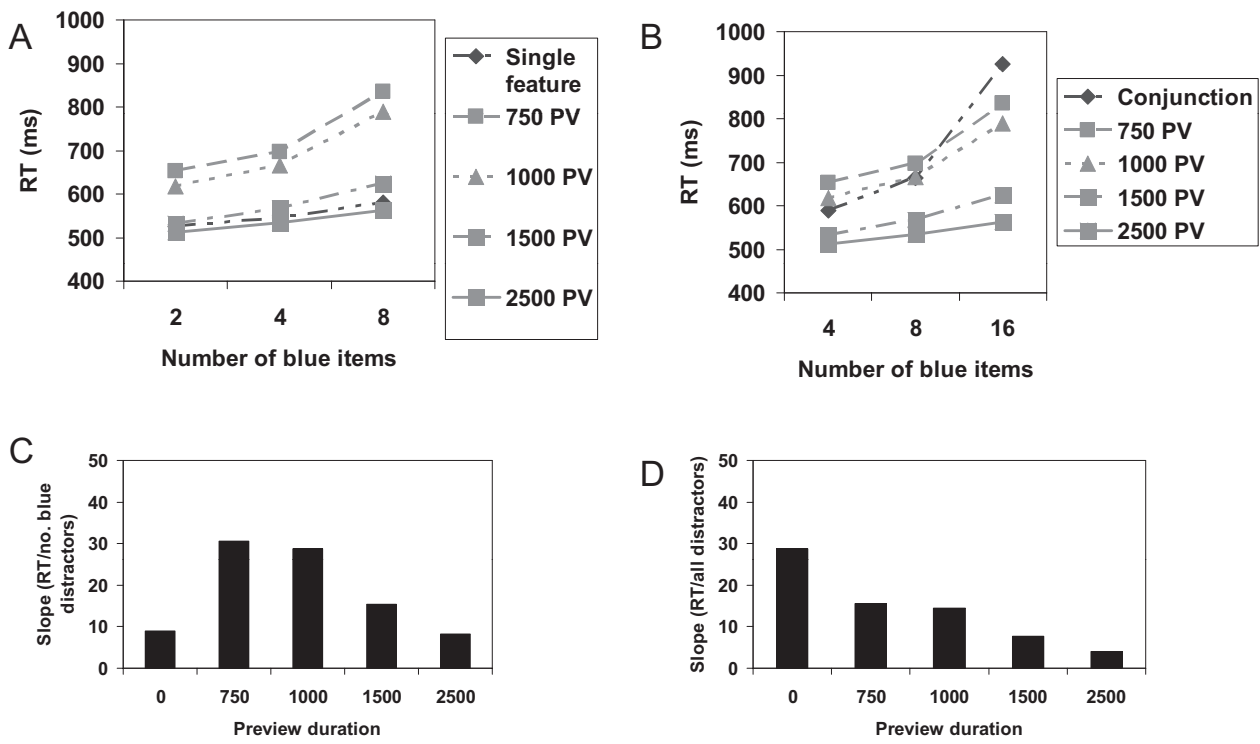

Figure 27. The mean correct reaction times (RTs) and the slopes of the search functions in Study 5B. A: RTs as a function of the number of blue stimuli, for four preview (PV) durations and a single-feature search task. In each preview condition, there was a secondary task load imposed. B: The data for the same preview conditions in relation to the total number of items in the final search display, plus data for the conjunction baseline condition. C: The slope of the single-feature (zero preview duration) and preview conditions against the number of blue stimuli. D: The slope of the search functions for the conjunction condition (zero preview duration) and the preview conditions, measured relative to the total number of stimuli in the final search display.

conditions are plotted together (relative to the number of blue distractors), and in Figure $27 \mathrm{~b}$ the data are presented for the preview conditions relative to the conjunction condition (plots against the total number of distractors in the final search displays). The slopes for the functions, measured against the number of blue distractors or the total number of distractors, are shown in Figures $27 \mathrm{c}$ and $27 \mathrm{~d}$. In Figure $27 \mathrm{c}$ the zero preview duration is the single-feature baseline; in Figure 27D the zero preview condition is the conjunction baseline. If, in the preview condition, there is no impact of old green distractors, then search should be equivalent to that found in the single-feature baseline (in Figures 27A and 27C). Comparisons relative to the number of distractors in the final display (in Figures 27B and 27D) assess whether the preview improves search relative to when the initial display is absent (in the conjunction baseline).

The mean correct RTs were subjected to a two-factor repeatedmeasures ANOVA with the factors being condition (single feature, conjunction, and each preview condition) and display size (two, four, and eight, reflecting the number of blue distractors present). There were reliable main effects of condition and display size, $F(15,35)=75.23$, and $F(2,14)=340.24, \eta_{p}^{2}=.915$ and .98 , both $p$ s $<.001$, and a Condition $\times$ Display Size interaction, $F(10$, 70) $=32.3, \eta_{p}^{2}=.822, p<.001$.

The interaction was assessed by comparing each preview condition against the single-feature baseline. For the 750-, 1,000-, and 1,500-ms previews, the interactions between condition (preview vs. single feature) and display size were significant, $F(2,14)=$ $35.90,18.22$, and $5.19, \eta_{p}^{2}=.837, .722$, and .426 , respectively (all $p$ s $<.025)$. For the $2,500-\mathrm{ms}$ preview there was no effect of condition and no interaction with display size, $F(1,7)=3.27$, $\eta_{p}^{2}=.318, p>.05$, and $F<1.0$, respectively.

\section{Discussion}

The data show that, for preview durations of up to $1,500 \mathrm{~ms}$, there remained a cost in search efficiency compared with the single-feature baseline, when participants performed a secondary task during the preview period. However, at the longer preview duration $(2,500 \mathrm{~ms})$, this cost was completely eliminated. The eradication of the secondary task cost is unlikely to be due to participants having time to read the target number into short-term memory at the longest preview duration, which could lessen the secondary task load. We separated out trials where the secondary task target was present and when it was absent, because in the latter condition participants would have to remain monitoring the numbers at fixation throughout the preview period. There was no effect of the presence of the secondary task target and no interactions (all $F \mathrm{~s}<1.0$ ). We conclude that the effects of the secondary task decrease if there is a sufficiently long preview duration. This matches Prediction 2 from sSoTS, which holds that there is a delay rather than an elimination of suppression under load conditions due to frequency adaptation to the preview. The data contradict the original proposal from Watson and Humphreys (1997) that, under conditions of reduced resources, there would be less suppression of the preview irrespective of the preview's duration.

\section{Study 5C: \\ Effects of Cognitive Load and Preview Top-Up}

The third prediction from sSoTS is that, even when a secondary task load is imposed across the duration of the preview leading up to visual search, there can be residual effects of having processed the preview earlier (in a top-up period). Furthermore, this can 
occur even when the initial preview duration itself is not sufficient to optimize search (see Figures 13 and 17). This was examined in Study 5C. In this experiment, participants received preview durations of $750,1,000$, and $1,500 \mathrm{~ms}$, during which time they also carried out a secondary task (monitoring for a target in a stream of numbers). In Study 5B, we demonstrated that performance in the preview condition did not reach the single-feature baseline level with those durations. However, in contrast to the earlier study, we introduced a top-up preview of $350 \mathrm{~ms}$ prior to the secondary task starting. This duration preview typically does not optimize search performance (Humphreys, Jung Stalmann, \& Olivers, 2004). We assessed whether, even though insufficient in its own right, the top-up preview "pushed" processing of a prolonged preview into its adaptation state, to generate maximum efficiency in the preview task.

\section{Method}

Unless otherwise mentioned, the method was the same as for Study 5B. There were eight participants (aged 19-26 years, six female and two male). The preview conditions were altered so that a preview of 350-ms duration was inserted after fixation and prior to the start of the preview + load displays. There were three durations of preview where the secondary task load was present: $750,1,000$, and $1,500 \mathrm{~ms}$. One other change was that the fixation times for the single-feature and conjunction baselines were 1,500 ms. This provided a warning interval between that in the $350+$ 1,000 - and $350+1,500-\mathrm{ms}$ preview conditions.

\section{Results}

Errors were again infrequent, and there was no evidence of any speed-accuracy trade-offs. These data are presented in Table 2, and they were not analyzed further. The RT results are presented in Figure 28.

The RTs were analyzed as for Study $5 \mathrm{~B}$, assigning all the conditions to a single factor (condition), with display size nested within this. There were reliable main effects of condition and display size, $F(4,28)=41.90$, and $F(2,14)=103.56, \eta_{p}^{2}=.857$ and .937 , respectively, both $p \mathrm{~s}<.001$. There was also a Condition $\times$ Display Size interaction, $F(8,56)=17.84, \eta_{p}^{2}=.718, p<$ .001 .

Comparisons were made between each preview condition and the single-feature baseline. For the $350+750$-ms preview condition there was a reliable interaction between the condition and the display size, $F(2,14)=10.03, p<.01, \eta_{p}^{2}=.589$. For the other preview conditions $(350+1,000$ and $350+1,500)$ neither the main effect of condition (vs. the single-feature baseline) nor the Condition $\times$ Display Size interaction was reliable $(F<1.0$ for both interactions).

\section{Discussion}

A preview presented for $350 \mathrm{~ms}$ prior to a search display, without any imposed secondary task, is suboptimal for search (Humphreys, Jung Stalmann, \& Olivers, 2004; Watson \& Humphreys, 1997). Presenting a preview for this duration and then withdrawing attention through a secondary task, while leaving the
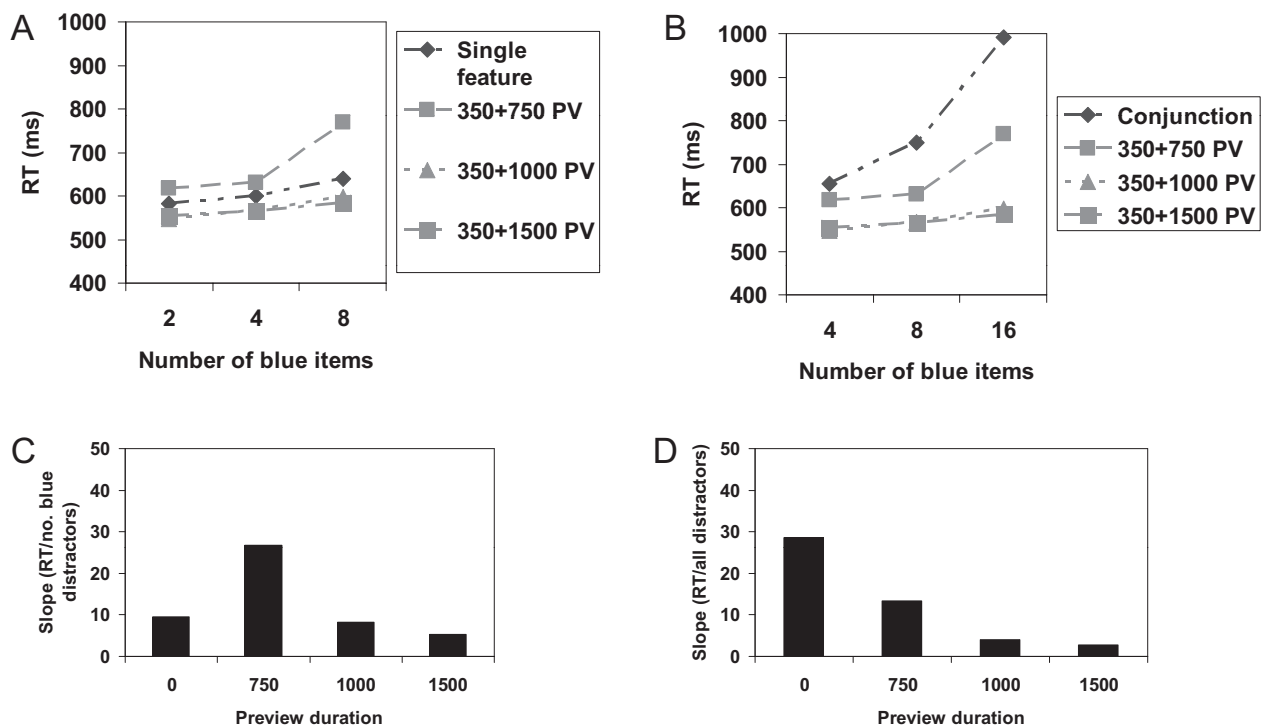

Figure 28. The mean correct reaction times (RTs) and the slopes of the search functions in Study 5C. A: RTs as a function of the number of blue stimuli, for three preview (PV) conditions and a single-feature search task. In the preview conditions there was a 350-ms standard preview followed by either a 750-, 1,000-, or 1,500-ms preview when a secondary task was also imposed. B: The data for the same preview conditions in relation to the total number of items in the final search display, plus data for the conjunction baseline condition. C: The slope of the single-feature (zero preview duration) and preview conditions against the number of blue stimuli. D: The slope of the search functions for the conjunction condition (zero preview duration) and the preview conditions, measured relative to the total number of stimuli in the final search display. 
preview present, disrupts subsequent search. This matches data reported by Humphreys et al. (2002) and Watson and Humphreys (1997). The new result here is that, with exactly the same duration of initial preview, we showed that search did approximate the efficiency levels in the single-feature baseline if the subsequent dual-task preview period was lengthened. This again indicates that withdrawing attentional resources, by engaging participants in a secondary task, is not sufficient to eradicate the preview benefit. The results fit with the predictions of the sSoTS model, where the initial top-up preview is sufficient to push activation toward an adaptation state, which is subsequently reached even when a secondary task is imposed during the following preview period. Once the preview enters this adaption state, an optimal preview benefit can arise.

\section{Study 5D: The Time Course of Preview Search After Lesioning}

When effects of a secondary task load are simulated, the sSoTS model generates efficient preview search but needs a prolonged preview period (see Figure 16). This picture contrasts with the data when sSoTS was lesioned. After lesioning the model showed relatively slow selection of targets falling on the affected side even across long durations of preview. We attribute this result to reduced data-driven activation on the affected side, which slows any accumulation of information and any subsequent adaptation. In Study 5D we assessed whether patients with PPC damage, matching the lesioning of the location map in sSoTS, would also show a residual deficit for contralesional targets in preview search.

\section{Method}

The method was the same as that in Study 5B except where otherwise mentioned. We tested six patients with damage focused around the right TPJ (patients JB, MC, TM, MP, RP, and BS; see Figure 25 and Table 1). Previews were presented for 1,000, 1,500, and $2000 \mathrm{~ms}$, and followed by the search displays for an unlimited period. In addition to this, blocks of single-feature and conjunction search trials were run. Unlike previously, the patients had to make a mouse click response when they detected the target. When this response was made, each occupied location in the search display was filled with a gray circle to cover the search letters. The patients were then asked to move the mouse to the location where they perceived the target. This procedure was adopted so that any deficits with contralesional targets were not due to slowed initiation of a response to the affected side (because an initial simple detection response was required). Targets appeared equally often in the left and right fields, and there were 96 trials per condition (16 per field per display size), making 480 trials in total. The patients performed the different trial blocks over periods of 2-3 weeks, depending on the patient.

\section{Results}

The accuracy and error data were combined to create a single efficiency score (RT/proportion correct; see Townsend \& Ashby, 1983). The data are plotted in Figure 29, showing RTs for the preview conditions against the single-feature baseline (top) and the conjunction baseline (bottom), assessing performance, respec- tively, against the number of blue items and the total number of items in the final search display. The slopes of the search functions, plotted against the number of blue items (in Panel a) and the total number of items (in Panel b), are shown in Figure 30.

Search efficiency was analyzed in a three-factor repeatedmeasures ANOVA with the factors condition (three preview conditions plus single-feature and conjunction baselines), visual field of target (contra- vs. ipsilesional), and display size (two or four blue items). There were reliable main effects of condition, visual field, and display size, $F(4,20)=18.94, F(1,5)=25.19$, and $F(1$, $5)=30.13, \eta_{p}^{2}=.791, .834$, and .858, respectively, all $p \mathrm{~s}<.001$. All the interactions were reliable, including the three-way interaction between condition, field, and display size, $F(4,20)=6.86$, $p<.001, \eta_{p}^{2}=.578$.

The three-way interaction was broken down by comparing the preview conditions against the single-feature and then the conjunction baseline. For each comparison between the preview and the single-feature condition, there was a significant interaction between condition (preview vs. single feature), field, and display size: for 1,000, 1,500, and $2000 \mathrm{~ms}, F(1,5)=7.14,8.17$, and 8.04, $\eta_{p}^{2}=.588, .62$, and .62, all $p \mathrm{~s}<.05$, respectively. In each case, the slope of the search function was steeper for the preview relative to the single-feature baseline, particularly for contralesional targets (see Figure 30).

Similar comparisons performed between the preview and conjunction conditions showed slightly different results across the different preview durations. For the 1,000 -ms preview there was no effect of condition, and the interaction with display size and field was not reliable, $F(1,5)=2.70, p>.05, \eta_{p}^{2}=.351$. For the 1,500- and 2,000-ms previews there were main effects of condition, $F(1,5)=19.12$ and 23.89, $\eta_{p}^{2}=.793$ and .83, both $p$ s $<.01$, but no interaction with field or display size. For the 2,000-ms preview the interaction between condition, field, and display size approached significance, $F(1,5)=4.41, p<.10, \eta_{p}^{2}=.468$. There was a trend for a lower slope for the preview than the conjunction condition, with the change being larger with contralesional targets.

\section{Discussion}

The data replicate prior studies of visual search in patients with damage to the PPC. There was relatively poor search for contralesional targets, with the effect tending to be more pronounced for conjunction than single-feature-defined stimuli (the slope for contralesional conjunction targets here was $217 \mathrm{~ms} / \mathrm{item}$, vs. $41 \mathrm{~ms} /$ item for the single-feature condition; see Eglin et al., 1990; Riddoch \& Humphreys, 1987). In addition, and again replicating prior data, the PPC patients were selectively impaired at preview search and indeed showed little preview benefit overall compared with the conjunction baseline (in terms of search efficiency). Agematched control participants showed asymptotic search performance, with little difference in slope for preview and singlefeature baselines, for preview durations of $750 \mathrm{~ms}$ or so (see Olivers \& Humphreys, 2004). In contrast, even with preview durations more than twice as long as this, the patients were still relatively impaired in the preview condition. There was a trend for some benefit emerging (compared with the conjunction baseline) at the longest preview duration (see Figures 29 and 30), but even then there was a cost to preview search against the single-feature 


\section{Contralesional target}

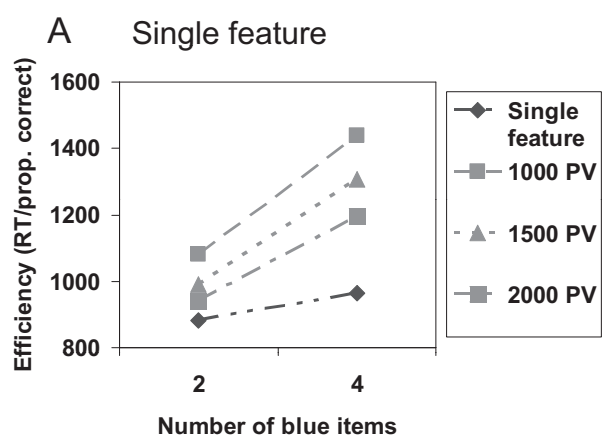

B Conjunction

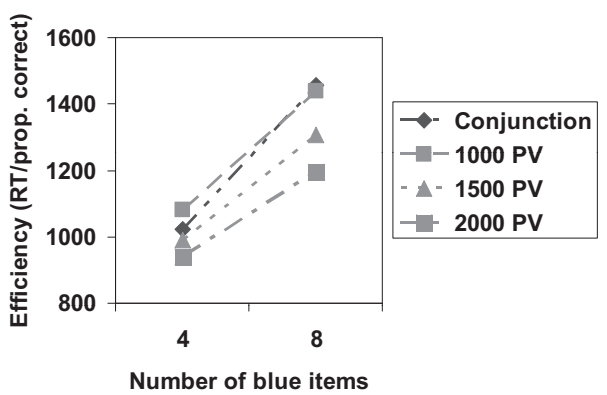

Ipsilesional target
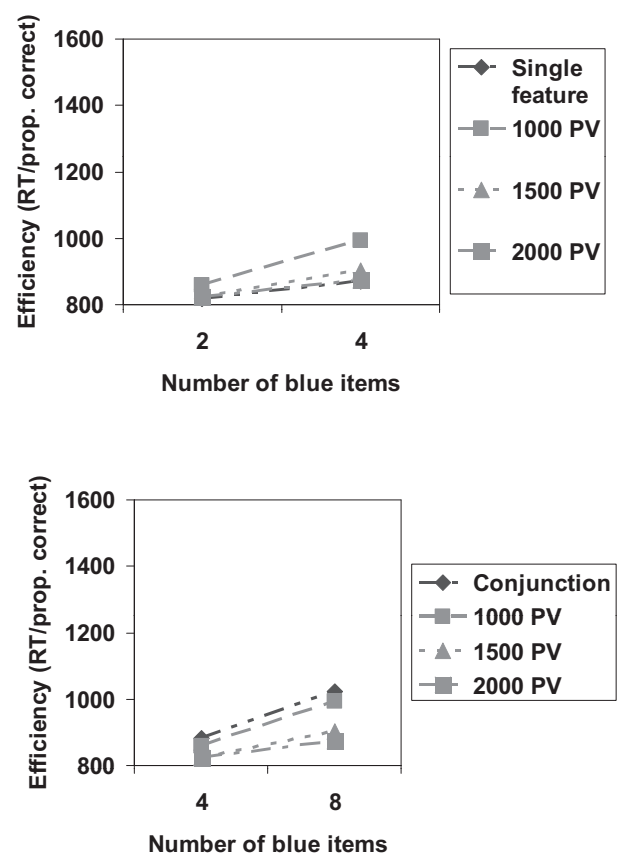

Figure 29. Search efficiency (RT/proportion correct) for a group of right posterior parietal cortex (PPC) patients in Study 5D. A: The data against the number of blue items for the single-feature baseline and preview (PV) search with three preview durations, as a function of whether the target fell in the contra- or ipsilesional field. B: The results for the same preview conditions along with a conjunction condition, in this case plotted against the total number of items in the final search display. RT = reaction time.

condition. We conclude that damage to the PPC makes it difficult for any adaptation effects to emerge under preview conditions. This matches the results from sSoTS and occurs in the model when lesions to the location map mean that the rate of gain in the affected units is insufficient to benefit from any boost coming from top-down biases under preview conditions (for target features and against distractor features).

\section{Study 5E: Predictions for fMRI}

The final prediction derived from sSoTS concerned the brain activation that we may expect according to whether preview search is conducted under conditions of secondary task load. Under conditions of task load, the differential activation within the location map for the target relative to distractors decreases (see Figure 24). Mavritsaki et al. (2010) have shown that there is a correlation between the HDR function predicted from the time course of activity in the location map and activity in the right TPJ in preview search. If this neural activity reflects the contrast between the target and distractors (i.e., the saliency of the target), then we predict that, compared with standard preview search, activity in the right TPJ should decrease under conditions of secondary task load. Allen et al. (2008) measured BOLD activity using fMRI for both standard preview search and for preview search conducted when participants were holding a load in working memory. Here we returned to these data and extracted activity within the right TPJ region found to correlate with the HDR derived from the location map in sSoTS, to test whether this region did show decreased activity under load conditions.

\section{Method}

The experiment and original data analysis were fully reported by Allen et al. (2008). In the basic preview condition, participants saw a set of blue faces (the preview, for $2 \mathrm{~s}$ ) followed by a search display of red house distractors and a blue house target. The task was to localize the target (left or right of fixation). In the dual-task condition, participants saw a random display of dots for $500 \mathrm{~ms}$ prior to the preview and search displays. They were instructed to keep the dot pattern in working memory during the preview display. For the current analysis, we selected a region of interest (ROI) based on the area found to correlate with the HDR function derived from the model's location map activity with a $z$ score $>3$. We extracted time courses of activation from this area for the preview and the preview + load conditions in Allen et al. and analyzed the data on the basis of the mean activity within the region in the two conditions (although the same results were also obtained using the peak activity; see Figure 31 ).

\section{Results}

The results are presented in Figure 31. There was a significant decrease in activation within the right TPJ in the preview + load 


\section{Contralesional target}

\section{A Single feature}

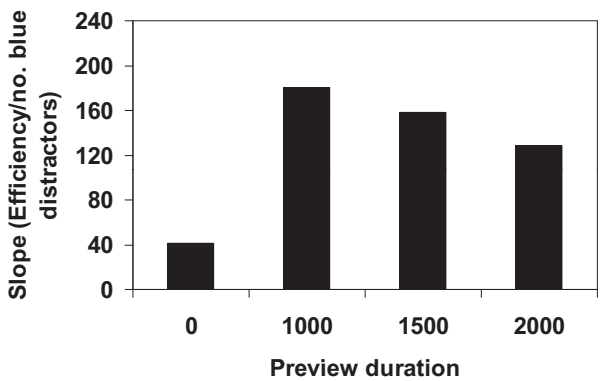

B Conjunction

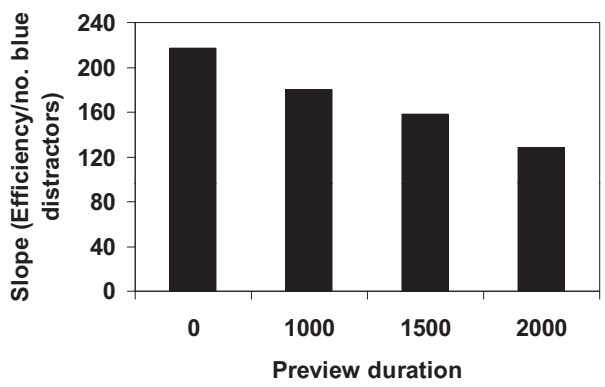

Ipsilesional target
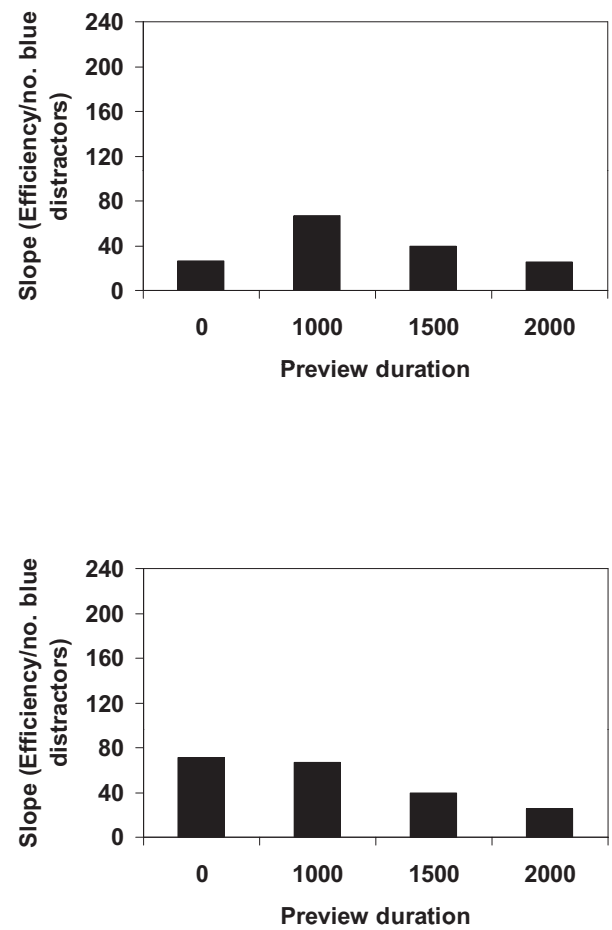

Figure 30. The slopes of the search functions for the right posterior parietal cortex (PPC) patients in Study 5D. A: The slopes for the single-feature condition (zero preview duration) and three preview duration conditions, measured against the number of blue items. B: The slopes for the conjunction condition (zero preview duration) along with the same preview conditions, shown relative to the total number of items in the final search display.

condition compared with the standard preview condition, $F(1$, 8) $=5.804, p<.05, \eta_{p}^{2}=.42$.

\section{Discussion}

The data match the prediction derived from sSoTS, if the right TPJ region reflects the saliency of targets (determined by the contrast between their activation and the activation of distractors): There was decreased activation in the preview + load condition compared with the standard preview search task. This result was not due to there being a general decrease in activation in the preview + load condition. For example, Allen et al. (2008) reported that this condition led to increased activation in the cuneus and precuneus, regions that were also found to be activated when participants ignored previews (even in a dummy preview condition, where search displays were not presented but participants prepared to ignore the preview). Allen et al. proposed that, in both the preview and the load task, participants hold a working memory representation, though in the case of preview search they suppress this representation (the preview) to help bias search to new stimuli. Critically, any increased activation due to the dot working memory load did not lead to activation of the right TPJ. This fits with the proposal that the right TPJ responds to the presence of salient, behaviorally relevant stimuli (here, the target, relative to the dis- tractors), perhaps to provide a signal to other response-related regions of cortex (see Corbetta et al., 2000). The saliency signal carried by the right TPJ may reflect either enhanced activity for the target or decreased activation/filtering of distractors mediated by this brain region. In either case, the data suggest that saliency decreased when a working memory load was held during the search task.

\section{General Discussion}

We have presented an argument that computational modeling within a biologically plausible framework can provide a useful means of linking neurophysiological studies of information processing through to whole-system behavior. Our argument has been based around the example of modeling human visual selection, where, using a consistent system architecture, we have shown that data at a number of neuroscientific levels can be simulated across a number of different neuroscientific levels: from single-cell electrophysiology (Study 1) through to whole-system behavioral experiments on visual search (Study 2), including also work on neuropsychological lesions of particular cortical regions (Study 3) and modeling fMRI data (Study 4). We have also argued that modeling using biologically plausible architectures can emphasize factors that are stressed less in other approaches, including the 


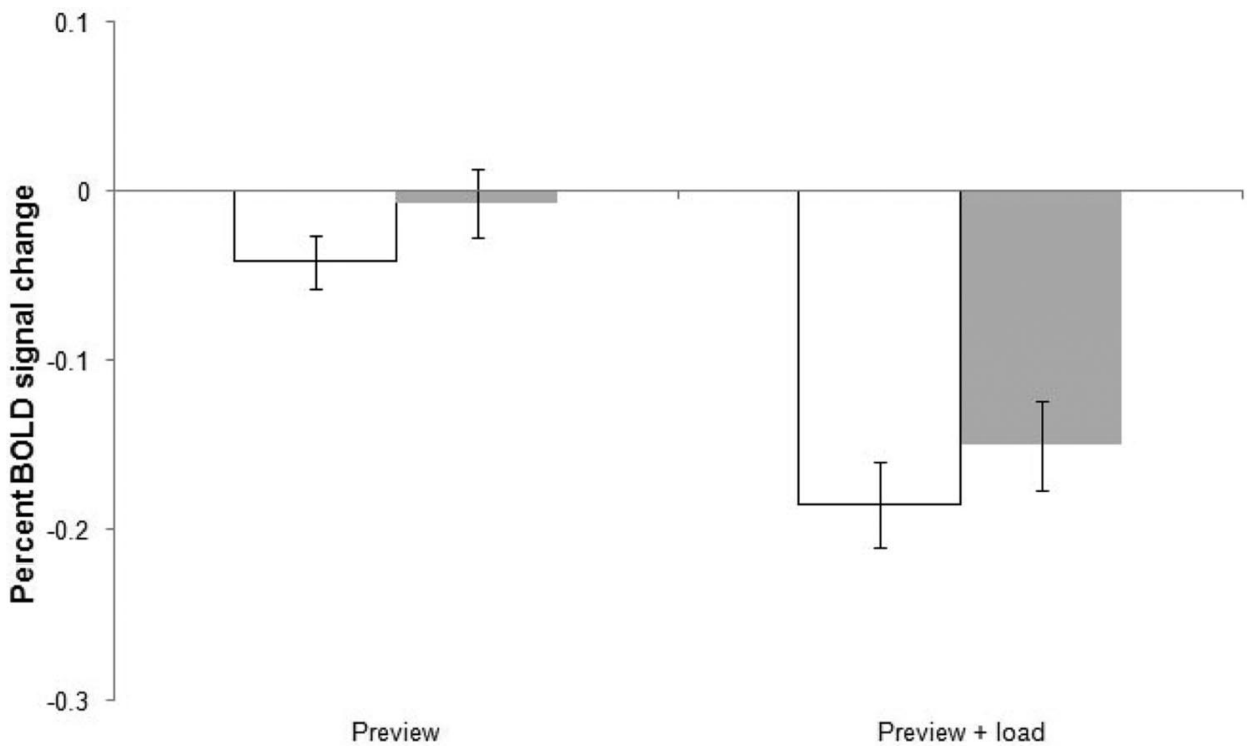

Figure 31. The percentage blood-oxygen level-dependent (BOLD) signal change in a region of interest (ROI) around the right temporoparietal junction (TPJ), based on the area that showed significant correlation with the hemodynamical response (HDR) time function derived from the location map of the spiking selection over time and space (sSoTS) model in preview search. Here the data are shown for the mean signal change across the ROI (white) or for the max signal change (gray). The results are for standard preview search and for preview search under secondary task conditions (preview + load). The error bars represent $1 S E$.

effects of noise/variability on information processing, the time course of processing, and the effects of neurotransmitter modulation/arousal on performance. Furthermore, novel predictions emerge due to models stressing these factors, and these predictions have been tested empirically (Study 5). Our data here show the following:

(a) Patients with right PPC damage are impaired at selecting stimuli presented vertically, compared with patients with posterior brain lesions not involving this area. This deficit can be reduced by using a cue to increase the patients' arousal. The data here match the argument that low arousal impairs visual selection even when spatial deficits are not apparent (Studies 1 and 5A);

(b) Under dual-task conditions, normal participants can show a normal preview benefit (with search slopes reaching the level found in single-feature search), provided preview exposures are sufficiently long. In sSoTS this result arises because the dual task causes delays in the time when preview adaptation takes places, but it does not eradicate it (Studies 2 and 5B);

(c) Giving an initial (top-up) preview prior to engaging participants in a secondary task can lead to preview benefit. This occurs even when there is a lack of a full preview benefit from either top-up alone or the preview under secondary task conditions. The predictions of the model here match data from sSoTS. In the model the result occurs because the initial preview pushes activation toward a level where adaptation can take place under dual-task conditions (Studies 2 and 5C);

(d) Patients with right PPC damage are impaired in preview search and do not reach levels of search efficiency found in the single-feature baseline even with prolonged presentations of previews. In sSoTS this result occurs because lesioning reduces activity within the location map so that there is continued compe- tition across an abnormally long time course (Studies 3 and 5D); and

(e) There is reduced activation within the right TPJ when preview search is undertaken under conditions of dual-task load. This is consistent with activation in the right TPJ reflecting the contrast of activation with the location map of sSoTS between target and distractor stimuli (Studies 4 and 5E).

Our stress here is that the predictions we have tested arise naturally from biologically plausible assumptions incorporated into sSoTS - from the role of neurotransmitter modulation (Study $5 \mathrm{~A}$ ), the temporal dynamics of processing and particularly the role of frequency adaptation (Studies 5B and 5C), the effects of reducing the neuronal population (Study 5D), and the predicted HDR function derived from synaptic transmission in the model (Study $5 \mathrm{E}$ ). This is not to state that these predictions could not be derived from other higher level models - it is certainly possible that they could. However, our contention is that the predictions follow from our attempt to use biologically plausible parameters, and the tests of the predictions provide a way to link lower level physiological processes (e.g., frequency adaptation) to whole-system behavior, typically examined in our search tasks. Overall, the results indicate that there can be a close interlinking between modeling and empirical investigations, with models providing the bridge between, for example, behavioral and imaging data.

The data we have reported highlight several factors that have not been demonstrated previously:

1. Modulating arousal levels (e.g., here, by giving a spatially uninformative auditory cue) can have a direct impact on the efficiency of visual selection (Studies 1 and 5A). In prior neuropsychological work it has been argued that decreased arousal is found after right PPC lesions in patients (Robertson \& Manley, 
1999), but this has been studied only within the context of the patients also showing a spatial deficit in selection. Our results indicate that selection deficits occur in these patients even when spatial biases in performance are eradicated under the presentation conditions. Our neural-level modeling provides a framework for understanding these nonspatial arousal effects.

2. The effects of a secondary task on preview search can be overcome over time. In earlier accounts of preview search (e.g., Watson \& Humphreys, 1997), it was argued that participants needed to fully engage attention in order to inhibit a preview display. Hence any removal of attentional capacity from this inhibition process would lead to a lessening of search efficiency. Our results indicate that this account is not correct. Even if a secondary task is imposed, preview search can be as efficient as a single-feature baseline (when only new search items appear) provided there is a sufficiently long preview. There are also effects of "topping up" a preview, and these effects are not eradicated as soon as a secondary task is imposed (Studies 5B and 5C). For the sSoTS model, these temporal effects arise due to the adaptation process, which operates when the preview reaches its threshold level of activation, irrespective of whether this threshold is passed with or without a second task. The effect of an initial top-up preview is also to push activity toward this threshold, giving rise to a benefit when the preview remains but now under dual-task conditions. The data highlight that the effects of time alone are important, even if a dual task disrupts top-down biases against distractors and toward targets. The exception to this, at least up to the time limits studied, occurs with right PPC patients, who remain unable to gain the maximum preview benefit even with prolonged preview displays (Study 5D). The contrast between the effects of secondary task load and PPC lesioning here is interesting. It suggests that the lesioning cannot be directly mimicked by giving normal participants a secondary task load. In sSoTS, this contrast reflects the difference between (a) a reduction in the rate of information accumulation across the field (under load conditions) and (b) the spatial imbalance of selection after a lesion to the location map and the slowed rate of information accumulation when location units are reduced in number. The spatial imbalance, and the slow accumulation of bottom-up activity in the location map, cannot be easily captured by a simple load manipulation.

3. In sSoTS, top-down inhibition is modulated by an initial enhancement of stimuli followed by inhibition of their features and locations. The process of initial attention, followed by subsequent inhibition, matches the probe report data of Humphreys, Jung Stalmann, and Olivers (2004). In this study the detection of probes falling on a preview was enhanced when the probes appeared shortly after the preview (200 ms), whereas detection appeared to be inhibited (relative to when the probe fell in a background location) when the delay was longer $(800 \mathrm{~ms})$. Inhibition in the model is applied across a feature map. This fits with data on preview search, where there are carry-over effects from old items to new targets sharing common features (e.g., see Braithwaite \& Humphreys, 2003; Olivers \& Humphreys, 2003). By placing old features into an inhibitory state, there will be delayed processing of targets if they are represented within the same feature maps.

4. The argument for initial enhancement of processing followed by inhibition, in sSoTS, fits with recent work showing that stimuli can be suppressed from awareness following an initial allocation of attention to their location (Carter, Luedeman, Mitroff, \& Nakayama, 2008), whereas sustained attention can reduce sensitivity to an attended stimulus (Ling \& Carrasco, 2006). In their fMRI study of preview search, Allen et al. (2008) and Payne and Allen (2010) also reported that there was initially enhanced activity in neural areas responding to the feature properties of previews followed by decreases in activity in the search period. This initial enhanced activity may reflect the initial allocation of attention to the preview. An alternative is that it could reflect the activation of inhibitory interneurons, which suppress preview-related activity. Against this last argument, other researchers have found either a decrease in BOLD activity to ignored distractors (Gazzaley, Cooney, McEvoy, Knight, \& D'Esposito, 2005; Smith, Singh, \& Greenlee, 2000) or no change in activation relative to baseline conditions (Egner \& Hirsch, 2005). However it also very difficult to know what to conclude from underactivity in BOLD data, because the findings may reflect the choice of baseline conditions rather than real reductions in neuronal responding. Interestingly, our analysis of brain areas linked to active suppression (Study 4) highlights the change in signal over time, rather than its reduction per se, and this form of model-based analysis may provide important converging evidence on the interpretation of any neuronal signals. The fact that excitatory and inhibitory activity were abstracted across different maps in sSoTS is also noteworthy. The data suggest that, at a neural level, particular regions of the PPC may be involved in coordinating inhibitory and excitatory guidance of selection, with the inhibitory and excitatory components pooling together to determine competition for selection in other brain regions. It will clearly be important to explore this possibility in more detail, using finer grained imaging protocols.

5. We have also shown how model-based analysis of fMRI data can suggest direct associations between localized components within a model (e.g., the location map in sSoTS) and localized brain regions (the right TPJ), providing converging evidence on the functional role of those regions. Here, our evaluation suggests that the right TPJ responds specifically to contrasts in activation between behaviorally relevant targets and behaviorally irrelevant distractors, and so indexes the salience of a target (Study 5E). We propose that this signal would be useful for other response systems, which care about the target's location but not necessarily about irrelevant parts of a scene. This argument is similar to the proposal made by Corbetta and Shulman (2002) that the right TPJ acts as a circuit breaker, signaling the presence of a potentially behaviorally relevant stimulus so that attention (including a saccadic eye movement) may be shifted to the selected location. Corbetta and Shulman speculated that the greater prevalence of visual neglect after damage to the right relative to the left PPC may reflect the lateralization of the circuit breaker to the right hemisphere. Our analysis is consistent with this, and it is noteworthy that we found the correlation with activity in the location map of sSoTS to be right lateralized, based on a whole-brain analysis. In addition to this, though, patients with right hemisphere lesions may also suffer due to a reduction in general arousal levels (Mavritsaki et al., 2009; Robertson et al., 1998). If this is coupled with a specific deficit in detecting contralesional, salience-defined targets (Studies 5D and 5E), then there will be poor selection of stimuli on the contralesional side of space. 


\section{Limitations of sSoTS}

The sSoTS model is what we might term a "minimal" model, concentrating on the time course of selection of a likely target location in search. The model does not realistically capture aspects of early visual processing, nor does it deal with how visual selection may link to ongoing selection of a response to a stimulus (e.g., to reach to or make a saccade to a target's position). Clearly, sSoTS will need to be extended to cover these other topics. Nevertheless, even within its limited domain, the model usefully shows how different patterns of search can emerge as the temporal properties of displays are varied and how neuropsychological deficits can stem naturally from reductions in the number of neurons supporting selection. The fits of the model to the data here are primarily qualitative, based on common patterns of performance as different factors are varied (e.g., the duration of a preview or the presence of a secondary task). On top of this, our work shows how quantitative variations in activation within a model can usefully complement brain imaging. For example, our model-based analysis uses quantitative fitting of activation patterns (and predicted HDR functions) to differentiate between (a) brain regions linked to the excitatory guidance of attention to targets and (b) the inhibitory suppression of irrelevant distractors (see Figure 22). This goes beyond simple contrasts between search conditions, where the contrast may involve both excitatory and inhibitory processes (e.g., contrasting preview with conjunction search; cf. Allen et al., 2008; Braithwaite \& Humphreys, 2003).

To date, the vast majority of attempts to develop biologically plausible models, operating at a neural level, have limited themselves to dealing with single domains, even to single experiments-one reason for this being the computational constraints involved in running larger scale simulations of this type, the other being that it is not always clear how to link physiological processes in the model to the larger scale emergent behavior of whole systems. One contribution of the sSoTS simulations presented here is to show that neural-level models can have a wide scope, with the same model covering single-cell electrophysiology, neuropsychological deficits, fMRI, and whole-system reaction times, and in doing this, the model provides a multilevel account of visual selection. Nevertheless it is moot question as to whether such models will scale up, as additional components are added-for example, the addition of a fully dynamic working memory system to modulate top-down biases, or the addition of a response selection system to guide action to a target's location. We note, however, the utility of mean field approximations here. Mean fieldlevel models operate above the spiking level but can then be "rolled out" at the spiking level. For instance, a mean field account of interactions between several modules can be tested not only by its overall, whole-system behavior, but also by (subsequently) focusing on spiking activity within just one level (where the more specific evidence exists). The direct linkage between mean fieldand spiking-level models provides a framework for developing larger scale models, which we look forward to exploring.

\section{References}

Agter, F., \& Donk, M. (2005). Prioritized selection in visual search through onset capture and color inhibition: Evidence from a probe-dot detection task. Journal of Experimental Psychology: Human Perception and Performance, 31, 722-730. doi:10.1037/0096-1523.31.4.722
Allen, H. A., \& Humphreys, G. W. (2007). Previewing distractors reduces their effective contrast. Vision Research, 47, 2992-3000. doi:10.1016/ j.visres.2007.07.019

Allen, H. A., Humphreys, G. W., \& Matthews, P. M. (2008). A neural marker of content-specific active ignoring. Journal of Experimental Psychology: Human Perception and Performance, 34, 286-297. doi: 10.1037/0096-1523.34.2.286

Braitenberg, V., \& Schüz, A. (1991). Anatomy of the cortex: Statistics and geometry (Vol. 18). New York, NY: Springer-Verlag.

Braithwaite, J. J., \& Humphreys, G. W. (2003). Inhibition and anticipation in visual search: Evidence from effects of color foreknowledge on preview search. Perception \& Psychophysics, 65, 213-237.

Braithwaite, J. J., Humphreys, G. W., \& Hulleman, J. (2005). Color-based grouping and inhibition in visual search: Evidence from a probedetection analysis of preview search. Perception \& Psychophysics, 67, 81-101.

Brunel, N., \& Wang, X. (2001). Effects of neuromodulation in a cortical network model of object working memory dominated by recurrent inhibition. Journal of Computational Neuroscience, 11, 63-85. doi: 10.1023/A:1011204814320

Bundesen, C. (1990). A theory of visual attention. Psychological Review, 97, 523-547. doi:10.1037/0033-295X.97.4.523

Bundesen, C., Habekost, T., \& Kyllingsbaek, S. (2005). A neural theory of visual attention: Bridging cognition and neurophysiology. Psychological Review, 112, 291-328. doi:10.1037/0033-295X.112.2.291

Carter, O., Luedeman, R., Mitroff, S., \& Nakayama, K. (2008). Motion induced blindness: The more you attend the less you see. [Abstract]. Journal of Vision, 8, 237. doi:10.1167/8.6.237

Corbetta, M., Kincade, J. I. M., Olinger, J. I. M., McAvoy, M. P., \& Shulman, G. L. (2000). Voluntary orienting is dissociated from target detection in human posterior parietal cortex. Nature Neuroscience, 3, 292-297. doi:10.1038/73009

Corbetta, M., \& Shulman, G. L. (2002). Control of goal-directed and stimulus-driven attention in the brain. Nature Reviews Neuroscience, 3, 201-215. doi:10.1038/nrn755

Corchs, S., \& Deco, G. (2004). Feature-based attention in human visual cortex: Simulation of fMRI data. NeuroImage, 21, 36-45. doi:10.1016/ j.neuroimage.2003.08.045

Deco, G., \& Rolls, E. (2005a). Neurodynamics of biased competition and cooperation for attention: A model with spiking neurons. Journal of Neurophysiology, 94, 295-313. doi:10.1152/jn.01095.2004

Deco, G., \& Rolls, E. (2005b). Sequential memory: A putative neural and synaptic dynamical mechanism. Journal of Cognitive Neuroscience, 17, 294-307. doi: $10.1162 / 0898929053124875$

Deco, G., Rolls, E., \& Horwitz, B. (2004). Integrating fMRI and single-cell data of visual working memory. Neurocomputing, 58-60, 729-737. doi:10.1016/j.neucom.2004.01.120

Deco, G., \& Zihl, J. (2001). Top-down selective visual attention: A neurodynamical approach. Visual Cognition, 8, 118-139. doi:10.1080/ 13506280042000054

Duncan, J., Humphreys, G. W., \& Ward, R. (1997). Competitive brain activity in visual attention. Current Opinion in Neurobiology, 7, 255261. doi:10.1016/S0959-4388(97)80014-1

Durstewitz, D., Seamans, J. K., \& Sejnowski, T. J. (2000). Neurocomputational models of working memory. Nature Neuroscience, 3, 1184 1191. doi: $10.1038 / 81460$

Eglin, M., Robertson, L. C., \& Knight, R. T. (1990). Visual search performance in the neglect syndrome. Journal of Cognitive Neuroscience, 1, 372-385. doi:10.1162/jocn.1989.1.4.372

Egner, T., \& Hirsch, J. (2005). Cognitive control mechanisms resolve conflict through cortical amplification of task-relevant information. $\mathrm{Na}$ ture Neuroscience, 8, 1784-1790. doi:10.1038/nn1594

Folk, C. L., Remington, R. W., \& Johnston, S. (1992). Involuntary covert orienting is contingent on attentional control settings. Journal of Exper- 
imental Psychology: Human Perception and Performance, 18, 10301044. doi:10.1037/0096-1523.18.4.1030

Found, A., \& Muller, H. J. (1996). Searching for features across dimensions: Evidence for a dimensional weighting account. Perception \& Psychophysics, 58, 88-101.

Gazzaley, A., Cooney, J. W., McEvoy, K., Knight, R. T., \& D’Esposito, M. (2005). Top-down enhancement and suppression of the magnitude and speed of neural activity. Journal of Cognitive Neuroscience, 17, 507517. doi:10.1162/0898929053279522

Gilzenrat, M. S., Holmes, B. D., Rajkowski, J., Aston-Jones, G., \& Cohen, J. J. (2002). Simplified dynamics in a model of noradrenergic modulation of cognitive performance. Neural Networks, 15, 647-663. doi: 10.1016/S0893-6080(02)00055-2

Glover, G. H. (1999). Deconvolution of impulse response in event-related BOLD fMRI. Neurolmage, 9, 416-429. doi:10.1006/nimg.1998.0419

Grossberg, S., \& Grunewald, A. (1997). Cortical synchronization and perceptual framing. Journal of Cognitive Neuroscience, 9, 117-132. doi:10.1162/jocn.1997.9.1.117

Heilman, K. M., \& Valenstein, E. (1979). Mechanisms underlying hemispatial neglect. Annals of Neurology, 5, 166-170. doi:10.1002/ ana. 410050210

Heinke, D., \& Humphreys, G. (2003). Attention, spatial representation and visual neglect: Simulating emergent attention and spatial memory in the selective attention for identification model (SAIM). Psychological Review, 110, 29-87. doi:10.1037/0033-295X.110.1.29

Heinke, D., Humphreys, G. W., \& Tweed, C. L. (2006). Top-down guidance of visual search: A computational account. Visual Cognition, 14, 985-1005. doi:10.1080/13506280500195482

Heinke, D., \& Mavritsaki, E. (2009). Bridging the gap between neurophysiology and behaviour. London, England: Psychology Press.

Humphreys, G. W. (1998). Neural representation of objects in space: A dual coding account. Philosophical Transactions of the Royal Society of London, Series B: Biological Sciences, 353, 1341-1351. doi:10.1098/ rstb. 1998.0288

Humphreys, G. W., Jolicoeur, P., \& Watson, D. (2002). Fractionating the preview benefit in search: Dual-task decomposition of visual marking by timing and modality. Journal of Experimental Psychology: Human Perception and Performance, 28, 640-660. doi:10.1037/00961523.28.3.640

Humphreys, G. W., Jung Stalmann, B., \& Olivers, C. N. L. (2004). An analysis of the time course of attention in preview search. Perception \& Psychophysics, 66, 713-730.

Humphreys, G. W., Kyllingsbaek, S., Watson, D. G., Olivers, C. N. L., Law, I., \& Paulson, O. (2004). Parieto-occipital areas involved in efficient filtering in search: A time course analysis of visual marking using behavioral and functional imaging procedures. Quarterly Journal of Experimental Psychology, 57(A), 610-635.

Humphreys, G. W., Mavritsaki, E., Allen, H. A., Heinke, D. G., \& Deco, G. (2009). Modelling visual search in biologically plausible neural networks: From whole system behaviour through neuropsychology to fMRI. In D. Heinke \& E. Mavritsaki (Ed.), Bridging the gap between neurophysiology and behaviour. London, England: Psychology Press.

Humphreys, G. W., Olivers, C. N. L., \& Braithwaite, J. J. (2006). The time course of preview search with color-defined, not luminance-defined, stimuli. Perception \& Psychophysics, 68, 1351-1358.

Humphreys, G. W., \& Riddoch, M. J. (1994). Attention to within-subject and between-object spatial representations: Multiple sites for visual selection. Cognitive Neuropsychology, 11, 207-241. doi:10.1080/ 02643299408251974

Itti, L., \& Koch, C. (2001). Computational modeling of visual attention. Nature Reviews Neuroscience, 2, 194-203. doi:10.1038/35058500

Ives, H. E. (1912). Studies of the photometry of different colours: I. Spectral luminosity curves obtained by equality of brightness photom- eter and the flicker photometer under similar conditions. Philosophical Magazine, 24, 149-188.

Jahr, C. E., \& Stevens, C. (1990). Voltage-dependence of NMDA-activated macroscopic conductances predicted by single-channel kinetics. Journal of Neuroscience, 10, 3178-3182.

Karnath, H. O. (1988). Deficits of attention in acute and recovered visual hemi-neglect. Neuropsychologia, 26, 27-43. doi:10.1016/00283932(88)90028-0

Kunar, M. A., Humphreys, G. W., \& Smith, A. C. (2003). History matters: The preview benefit in search is not onset capture. Psychological Science, 14, 181-185. doi:10.1111/1467-9280.t01-1-01439

Léveillé, J., Versace, M., \& Grossberg, S. (2010). Running as fast as it can: How spiking dynamics form object groupings in the laminar circuits of visual cortex. Journal of Computational Neuroscience, 28, 323-346. doi:10.1007/s10827-009-0211-1

Ling, S., \& Carrasco, M. (2006). When sustained attention impairs perception. Nature Neuroscience, 9, 1243-1245. doi:10.1038/nn1761

Liu, Y. H., \& Wang, X. (2001). Spike-frequency adaptation of a generalized leaky integrate-and-fire model neuron. Journal of Computational Neuroscience, 10, 25-45. doi:10.1023/A:1008916026143

Logothetis, N. K. (2008, June 12). What we can do and what we cannot do with fMRI. Nature, 453, 869-878.

Logothetis, N. K., Pauls, J., Augath, M. A., Trinath, T., \& Oeltermann, A. (2001, July 12). Neurophysiological investigation of the basis of the fMRI signal. Nature, 412, 150-157. doi:10.1038/35084005

Luce, R. D. (1959). Individual choice behavior. New York, NY: Wiley.

Luce, R. D. (1977). The choice axiom after twenty years. Journal of Mathematical Psychology, 15, 215-233. doi:10.1016/00222496(77)90032-3

Madison, D., \& Nicoll, R. (1984). Control of the repetitive discharge of rat CA 1 pyramidal neurones in vitro. Journal of Physiology, 345, 319-331.

Malhotra, P., Parton, A. D., Greenwood, R., \& Husain, M. (2005). Noradrenergic modulation of space exploration in visual neglect. Annals of Neurology, 59, 189-190.

Marr, D. (1982). Vision. San Francisco, CA: W. H. Freeman.

Martínez-Trujillo, J. C., \& Treue, S. (2002). Attentional modulation strength in cortical area MT depends on stimulus contrast. Neuron, 35, 365-370. doi:10.1016/S0896-6273(02)00778-X

Mattingley, J. B., Davis, G., \& Driver, J. (1997, January 31). Preattentive filling-in of visual surfaces in parietal extinction. Science, 275, 671-674. doi:10.1126/science.275.5300.671

Mavritsaki, E., Allen, H., \& Humphreys, G. W. (2008). Model based analysis of fMRI-data: Applying the sSoTS framework to the neural basis of preview search. In L. Paletta \& J. K. Tsotsos (Eds.), Lecture notes in artificial intelligence: Attention in cognitive systems (Vol. 5395, pp. 124-138). Berlin, Germany: Springer-Verlag.

Mavritsaki, E., Allen, H., \& Humphreys, G. W. (2010). Decomposing the neural mechanisms of visual search through model-based analysis of fMRI: Top-down excitation, active ignoring and the use of saliency by the right TPJ. NeuroImage, 52, 934-946. doi:10.1016/j.neuroimage.2010.03.044

Mavritsaki, E., Heinke, D., Deco, G., \& Humphreys, G. W. (2009). Simulating posterior parietal damage in a biologically plausible framework: Neuropsychological tests of the search over time and space model. Cognitive Neuropsychology, 26, 343-390. doi:10.1080/ 02643290903424329

Mavritsaki, E., Heinke, D., Humphreys, G. W., \& Deco, G. (2006). A computational model of visual marking using an interconnected network of spiking neurons: The spiking search over time \& space model (sSoTS). Journal of Physiology-Paris, 100, 110-124. doi:10.1016/ j.jphysparis.2006.09.003

Mehler, J., Morton, J., \& Jusczyk, P. W. (1984). On reducing language to biology. Cognitive Neuropsychology, 1, 83-116. doi:10.1080/ 02643298408252017

Moran, J., \& Desimone, R. (1985, August 23). Selective attention gates 
visual processing in the extrastriate cortex. Science, 229, 782-784. doi:10.1126/science.4023713

Mozer, M. C., Halligan, P. W., \& Marshall, J. C. (1997). The end of the line for a brain-damaged model of hemispatial neglect. Journal of Cognitive Neuroscience, 2, 96-123. doi:10.1162/jocn.1997.9.2.171

Mumford, D. (1992). On the computational architecture of the neocortex: II. The role of cortico-cortical loops. Biological Cybernetics, 66, 241251. doi:10.1007/BF00198477

Nieuwenhuis, S., Gilzenrat, M. S., Holmes, B. D., \& Cohen, J. D. (2005). The role of the locus coeruleus in mediating the attentional blink: A neurocomputational theory. Journal of Experimental Psychology: General, 134, 291-307. doi:10.1037/0096-3445.134.3.291

Olivers, C. N. L., \& Humphreys, G. W. (2003). Visual marking inhibits singleton capture. Cognitive Psychology, 47, 1-42. doi:10.1016/S00100285(03)00003-3

Olivers, C. N. L., \& Humphreys, G. W. (2004). Spatiotemporal segregation in visual search: Evidence from parietal lesions. Journal of Experimental Psychology: Human Perception and Performance, 30, 667-688. doi: 10.1037/0096-1523.30.4.667

Olivers, C. N. L., Smith, S., Matthews, P. M., \& Humphreys, G. W. (2005). Prioritizing new over old: An fMRI study of the preview search task. Human Brain Mapping, 24, 69-78. doi:10.1002/hbm.20071

Pashler, H. (2000). Task switching and multitask performance. In S. Monsell \& J. Driver (Eds.), Attention and Performance XVIII: Control of Cognitive Processes (pp. 277-308). London, England: Academic Press.

Payne, H. E., \& Allen, H. A. (2010). Active ignoring in early visual cortex. Journal of Cognitive Neuroscience. Advance online publication. doi: 10.1162/jocn.2010.21562

Pollmann, S., Weidner, R., Humphreys, G. W., Olivers, C. N. L., Muller, H. M., Lohmann, G., . . W Watson, D. G. (2003). Separating segmentation and target detection in posterior parietal cortex: An event-related fMRI study of visual marking. NeuroImage, 18, 310-323. doi:10.1016/S10538119(02)00036-8

Posner, M., \& Boies, S. J. (1971). Components of attention. Psychological Review, 78, 391-408. doi:10.1037/h0031333

Posner, M., \& Petersen, S. E. (1990). The attention system of the human brain. Annual Review of Neuroscience, 13, 25-42. doi:10.1146/ annurev.ne.13.030190.000325

Posner, M. I., Walker, J. A., Friedrich, F. J., \& Rafal, R. D. (1984). Effects of parietal injury on the covert orienting of attention. Journal of Neuroscience, 4, 1863-1874.

Pouget, A., \& Bavelier, D. (2007). Paying attention to neurons with discriminating taste. Neuron, 53, 473-475. doi:10.1016/j .neuron.2007.02.004

Ress, D., Backus, B., \& Heeger, D. (2000). Activity in primary visual cortex predicts performance in a visual detection task. Nature Neuroscience, 3, 940-945. doi:10.1038/78856

Reynolds, J. H., Chelazzi, L., \& Desimone, R. (1999). Competitive mechanisms subserve attention in macaque areas V2 and V4. Journal of Neuroscience, 19, 1736-1753.

Reynolds, J. H., \& Desimone, R. (2003). Interacting roles of attention and visual salience in V4. Neuron, 37, 853-863. doi:10.1016/S08966273(03)00097-7

Riddoch, M. J., \& Humphreys, G. W. (1987). A case of integrative visual agnosia. Brain, 110, 1431-1462. doi:10.1093/brain/110.6.1431

Riddoch, M. J., Humphreys, G. W., Edwards, S., Baker, T., \& Wilson, K. (2003). Seeing the action: Neuropsychological evidence for action-based effects on object selection. Nature Neuroscience, 6, 82-89. doi:10.1038/ nn984

Robertson, I. H., \& Manley, T. (1999). Sustained attention deficits in time and space. In G. W. Humphreys, J. Duncan, \& A. Treisman (Eds.), Attention, space and action: Studies in cognitive neuroscience (pp. 297-310). Oxford, England: Oxford University Press.
Robertson, I. H., Mattingley, J. B., Rorden, C., \& Driver, J. (1998, September 10). Phasic alerting of neglect patients overcomes their spatial deficit in visual awareness. Nature, 395, 169-172. doi:10.1038/25993

Roelfsema, P. R., Tolboom, M., \& Khayat, P. (2007). Different processing phases for features, figures, and selective attention in the primary visual vortex. Neuron, 56, 785-792. doi:10.1016/j.neuron.2007.10.006

Rolls, E., \& Treves, A. (1998). Neural networks and brain function. Oxford, England: Oxford University Press.

Schneider, W., Eschman, A., \& Zuccolotto, A. (2002a). E-Prime reference guide. Pittsburgh, PA: Psychology Software Tools.

Schneider, W., Eschman, A., \& Zuccolotto, A. (2002b). E-Prime user's guide. Pittsburgh, PA: Psychology Software Tools.

Shulman, G. L., Astafiev, S. V., McAvoy, M. P., Davossa, G., \& Corbetta, M. (2007). Right TPJ deactivation during visual search: Functional significance and support for a filter hypothesis. Cerebral Cortex, 17, 2625-2633. doi:10.1093/cercor/bhl170

Smith, A. T., Singh, K. D., \& Greenlee, M. W. (2000). Attentional suppression of activity in the human visual cortex. NeuroReport, 11, 271-278. doi:10.1097/00001756-200002070-00010

Stemme, A., Deco, G., Busch, A., \& Schneider, W. A. (2005). Neurons and the synaptic basis of the fMRI signal associated with cognitive flexibility. NeuroImage, 26, 454-470. doi:10.1016/j.neuroimage.2005.01.044

Sternberg, S. (1969). The discovery of processing stages: Extensions of Donders' method. Acta Psychologica, 30, 276-315. doi:10.1016/00016918(69)90055-9

Townsend, J. T. (1972). Some results concerning the identifiability of parallel and serial processes. British Journal of Mathematical and Statistical Psychology, 25, 168-199.

Townsend, J. T., \& Ashby, F. G. (1983). The stochastic modeling of elementary psychological processes. New York, NY: Cambridge University Press.

Treisman, A. M., \& Gelade, G. (1980). A feature-integration theory of attention. Cognitive Psychology, 12, 97-136. doi:10.1016/00100285(80)90005-5

Tsotsos, J. K., Culhane, S. M., Wai, W., Davis, N., \& Nuflo, F. (1995). Modeling visual attention via selective tuning. Artificial Intelligence, 78 , 507-545. doi:10.1016/0004-3702(95)00025-9

Usher, M., Cohen, J. D., Servan-Schreiber, D., Rajkowski, J., \& AstonJones, G. (1999, January 22). The role of locus coeruleus in the regulation of cognitive performance. Science, 283, 549-554. doi:10.1126/ science.283.5401.549

Usher, M., \& Davelaar, E. J. (2002). Neuromodulation of decision and response selection. Neural Networks, 15, 635-645. doi:10.1016/S08936080(02)00054-0

Usher, M., \& McClelland, J. L. (2001). The time course of perceptual choice: The leaky, competing accumulator model. Psychological Review, 108, 550-592. doi:10.1037/0033-295X.108.3.550

Ward, R., Goodrich, S., \& Driver, J. (1994). Grouping reduced visual extinction: Neuropsychological evidence for weight-linkage in visual selection. Visual Cognition, 1, 101-129. doi:10.1080/ 13506289408402295

Watson, D. G., \& Humphreys, G. W. (1997). Visual marking: Prioritizing selection for new objects by top-down attentional inhibition. Psychological Review, 104, 90-122. doi:10.1037/0033-295X.104.1.90

Watson, D. G., \& Humphreys, G. W. (2000). Visual marking: Evidence for inhibition using a probe-dot detection paradigm. Perception \& Psychophysics, 62, 471-481.

Watson, D. G., Humphreys, G. W., \& Olivers, C. (2003). Visual marking: Using time in visual selection. Trends in Cognitive Sciences, 7, 180186. doi:10.1016/S1364-6613(03)00033-0

Weidner, R., \& Muller, H. J. (2009). Dimensional weighting of primary and secondary target-defining dimensions in visual search for singleton conjunction targets. Psychological Research, 73, 198-211.

Wilson, F. A., O'Scalaidhe, S., \& Goldman-Rakic, P. (1994). Functional 
synergism between putative gamma-aminobutyrate-containing neurons in pyramidal neurons in prefrontal cortex. Proceedings of the National Academy of Sciences, USA, 91, 4009-4013. doi:10.1073/pnas.91.9.4009 Wilson, H., \& Cowan, S. (1972). Excitatory and inhibitory interactions in localized populations of model neurons. Biological Cybernetics, 12, 1-24. Wolfe, J. W. (1994). Guided search 2.0: A revised model of visual search. Psychonomic Bulletin \& Review, 1, 202-238.
Yantis, S., \& Gibson, B. S. (1994). Object continuity in apparent motion and attention. Canadian Journal of Experimental Psychology, 48, 182204. doi:10.1037/1196-1961.48.2.182

Zheng, P., Zhang, X.-X., Bunney, B. S., \& Shi, W.-X. (1999). Opposite modulation of cortical $N$-methyl-D-aspartate receptor-mediated responses by low and high concentrations of dopamine. Neuroscience, 91, 527-535. doi:10.1016/S0306-4522(98)00604-6

\section{Appendix A}

\section{The Top-Down Inhibitory Function}

In the spiking selection over time and space (sSoTS) model, in addition to the passive adaptation process, there is also an active negative bias applied to distractors that are presented in advance of targets (e.g., under preview search conditions). This is implemented through a small increase to the outputs of the inhibitory pools to those feature maps representing the characteristics of the initial (previewed) distractors. Recent behavioral studies have indicated that stimuli can be suppressed from awareness following an initial allocation of attention to their location (Carter et al., 2008) but that sustained attention can impair sensitivity to an attended stimulus (Ling \& Carrasco, 2006). Matching these observations, we introduced a top-down negative bias based on the following function: AcIn $=\max A c * 100 *\left(e^{-500 / \text { time }}-0.99 e^{-500 / \text { time }+15)}\right)$. This function reduces the overall inhibition that the initially active feature maps receive in the first $300 \mathrm{~ms}$ of stimulus (preview) presentation. This enables these initial items to be fully attended before being inhibited. This matches data on preview search presented by Humphreys, Jung Stalmann, and Olivers (2004). The inhibitory function can be viewed in Figure 10, where we show the firing rates of the pool of neurons in the location map representing one of the previewed items for two cases: negative bias 0 and negative bias 0.18 . For negative bias 0 , the previewed item continues to maintain attentional salience for longer than when a negative bias of 0.18 is used (e.g., if the contrast between target and distractor activation in the location map reflects attentional salience).

\section{Appendix B}

\section{Calculating Search Slopes and Reaction Times}

The spiking level allows us to simulate reaction times (RTs) and target detection rates, with different runs of the model reflecting the Poisson noise present in neurons external to the model (e.g., responding to sensory input). As a consequence, runs with the same parameters provide a range of RTs, and there is a variable rate of detecting the target (selecting the units in the location map where the target falls).

To measure the reaction times and the detection rates, an attention index (IndA) is calculated based on Luce's (1959, 1977) choice axiom, $\operatorname{Ind} A(t)=\frac{f_{i}(t)}{\sum_{j} f_{j}(t)}$, where $f_{i}(t)$ represents the firing rate of pool $i$ at time $t$. The RT is determined when the attention index crosses a given threshold (set according to the average IndA for all conditions). The time that the threshold is crossed is considered the RT for the trial and, if the pool in the location map crossing the threshold first is the target pool, then we judge that the target has been detected successfully.

From the pool of parameters identified in mean field-level simulations, we selected one set of parameters that resulted in target selection for most values of $\mathrm{Ca}$. For this set of parameters, the behaviorally most plausible $\mathrm{Ca}$ parameter was then identified (see Figure 9C). Ideally, to fit with behavioral observations (e.g., Watson \& Humphreys, 1997), the slope for single-feature search should be below $19 \mathrm{~ms} /$ item and the slope for conjunction less than 40 but higher than $26 \mathrm{~ms} /$ item. Furthermore, the detection rate for conjunction search had to be above $80 \%$. All these criteria were satisfied when a Ca concentration of 0.2 was used (see Figure 9C). 


\section{Appendix C}

\section{Parameters Used in sSoTS}

\begin{tabular}{|c|c|c|}
\hline Parameter & Value & Description \\
\hline$g_{A M P A, r e c}$ excitatory & $0.0208 \mathrm{nS}$ & AMPA recurrent synaptic conductance for excitatory neurons \\
\hline$g_{A M P A, \text { rec }}$ inhibitory & $0.0162 \mathrm{nS}$ & AMPA recurrent synaptic conductance for inhibitory neurons \\
\hline$g_{N M D A}$ excitatory & $0.22 \mathrm{nS}$ & NMDA recurrent synaptic conductance for excitatory neurons \\
\hline$a$ & $0.18 \mu \mathrm{M}$ & {$\left[\mathrm{Ca}^{2+}\right]$ influx when a spike occurs } \\
\hline$N_{E}$ & $1,600(800)$ & $\begin{array}{l}\text { Number of excitatory neurons in each layer for the feature maps } \\
\text { (for the location map) }\end{array}$ \\
\hline$N_{I}$ & $400(200)$ & $\begin{array}{l}\text { Number of inhibitory neurons in each layer for the feature maps } \\
\text { (for the location map) }\end{array}$ \\
\hline$N_{e x t}$ & 800 & Number of external neurons \\
\hline$w^{+}$ & 2.2 & Coupling for the pools in the feature maps \\
\hline$w_{i 1}$ & 1.0 & Inhibition for the two feature dimension maps \\
\hline$w_{i 2}$ & 0.9 & Inhibition for the location map \\
\hline$w_{i 3}$ & 1.0 & Connection weight from feature maps to the location map \\
\hline$w_{i 4}$ & 0.25 & Connection weight from the location map to feature maps \\
\hline$\lambda_{\text {in }}$ & $150 \mathrm{~Hz}$ & $\begin{array}{l}\text { The total input that each pool receives from the external } \\
\text { neurons to show that there is an item in the visual field }\end{array}$ \\
\hline$\lambda_{\text {att }}$ & $190 \mathrm{~Hz}$ & $\begin{array}{l}\text { The total top-down that the target pools receive to signify the } \\
\text { target's characteristics }\end{array}$ \\
\hline $\max A c$ & 0.18 & $\begin{array}{l}\text { The maximum top-down inhibition that can be applied to the } \\
\text { previewed distractors' maps }\end{array}$ \\
\hline
\end{tabular}

Note. Parameters were set to generate data matching human search for single-feature, conjunction, and preview conditions (preview duration 1,000 ms). Subsequently, the only parameter variations that occurred reflected changes in the top-down parameters, presumed to occur under secondary task conditions. sSoTS $=$ spiking selection over time and space model.

Received December 15, 2008

Revision received July 29, 2010

Accepted July 30, 2010 